\title{
AL. $2.2002-126$
}

C. 2

\section{RANGE PLANT COMMUNITIES AND} CARRYING CAPACITY FOR THE UPPER FOOTHILLS SUBREGION OF ALBERTA
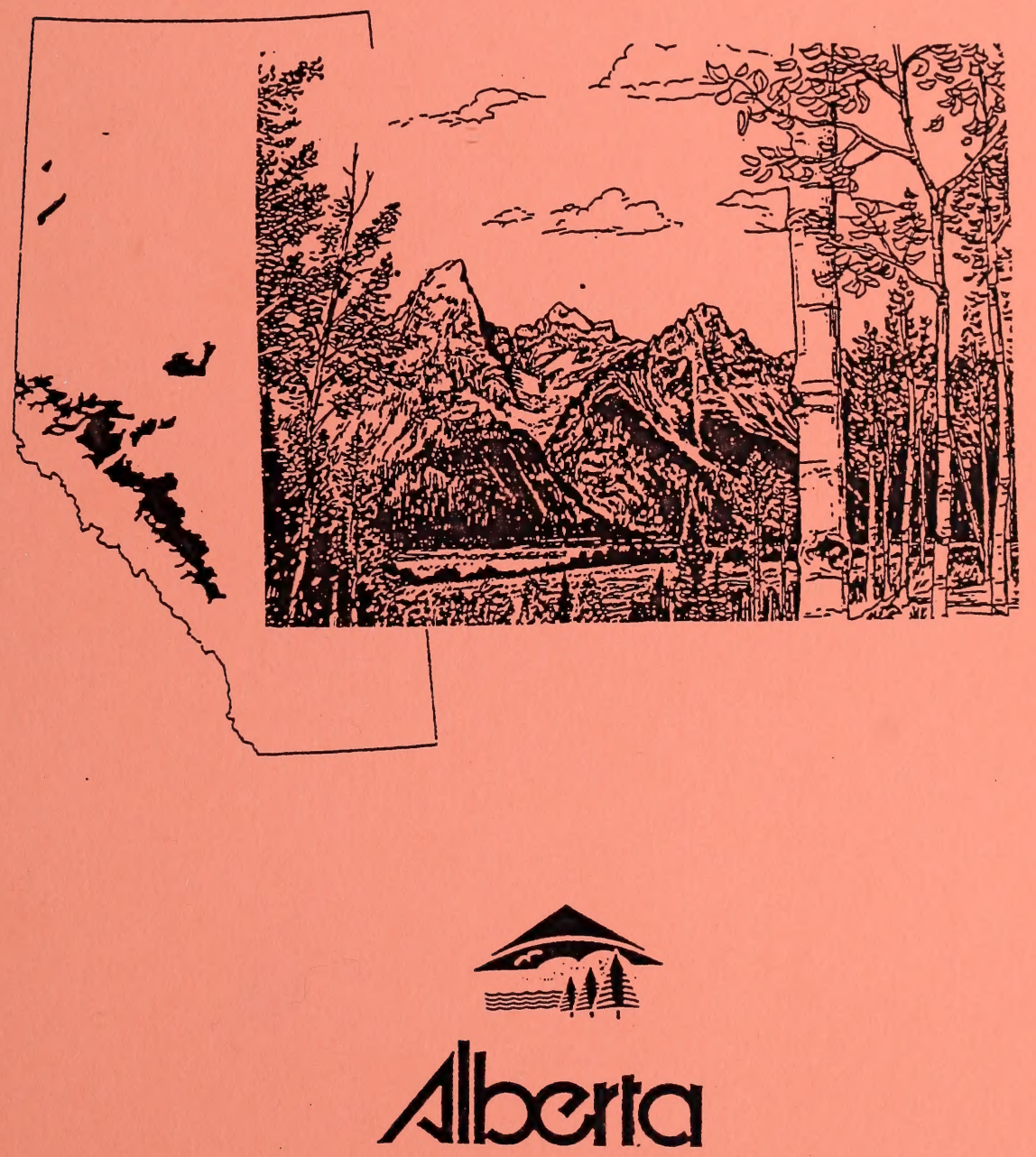

SUSTAINABLE RESOURCE DEVELOPMENT 



\section{RANGE PLANT COMMUNITY TYPES AND CARRYING CAPACITY FOR THE UPPER FOOTHILLS SUBREGION}

Fourth approximation

(This publication is a revision of the second and third approximation of the Range Plant

Communities and Carrying Capacity for the Upper Foothills Publication No. T/356)

2001

Prepared by

Michael G. Willoughby

Edmonton

2001
Alberta Sustainable Resource Development Public Lands Division 
Pub. no.: T/003

ISBN: 0-7785-1792-6

For copies of this report contact:

- Michael Willoughby 9920108 St., 9th Floor

Edmonton, Alta.

T5K 2M4

(403) $422-4598$

E-mail: mike.willoughby@gov.ab.ca 
Introduction

Climate of the Upper Foothills subregion

Methods

How to use this guide $\quad 4$

Results 5

$\begin{array}{ll}\text { Native grass and shrublands } & 31\end{array}$

Key to grasslands $\quad 36$

Key to modified grass and shrublands $\quad 37$

Key to native shrublands 38

A. Grasslands

UFA1. Water sedge meadows $\quad 39$

UFA2. Sedge-Slender wheatgrass/Meadow rue 40

UFA3. Tufted hairgrass-Sedge 41

UFA4. Tufted hairgrass-Sedge-Slender wheatgrass 42

UFA5. Rough fescue-Tufted hairgrass 43

UFA6. Rough fescue-Hairy wildrye 44

UFA7. Rough fescue/Bearberry 45

UFA7a.California oatgrass-Rough fescue/Bearberry 46

UFA8. California oatgrass-Sedge 47

UFA9. Junegrass/Sedge $\cdot 48$

UFA10. Bearberry/Slender wheatgrass 49

UFA11. Fireweed/Hairy wildrye(Forb meadow) - 50

UFA12. Rough fescue-Bog sedge 51

UFA13. Alpine rough fescue $\quad 52$

UFA14. Cow parsnip-Meadow rue/Fringed brome 53

UFA15. Hairy wildrye-Sedge 54

UFA16. Hairy wildrye-Rough fescue/Bearberry $\quad 55$

UFA17. Idaho fescue-Parry oatgrass-Sedge . 56 
B. Shrublands

UFB1. Willow-Bog birch/Water sedge $\quad 57$

UFB2. Willow/Slender wheatgrass-Sedge 58

UFB3. Willow/Tufted hairgrass $\quad 59$

UFB4. Willow/Rough fescue $\quad 60$

UFB5. Bog birch/Rough fescue/Bearberry 61

UFB6. Willow/Califomia oatgrass-Sedge $\quad 62$

UFB7. Pussy willow shrubland 63

UFB8. Willow/Hairy wildrye-Sedge $\quad 64$

UFB9. Bog birch/Sedge-Marsh reedgrass $\quad 65$

UFB10. Willow-Bog birch/Sedge 66

UFB11. Willow-Bog birch $\quad 67$

UFB12. Alder-Willow/Horsetail 68

C. Shrublands and Grasslands modified by grazing 69

UFC1. Slender wheatgrass-Sedge/Low forbs 72

UFC2. Rocky Mtn. fescue/Graceful cinquefoil 73

UFC3. Kentucky bluegrass/Dandelion-Clover 74

UFC4. Kentucky bluegrass-Sedge/Dandelion $\quad 75$

UFC5. Tufted hairgrass-Kentucky bluegrass 76

UFC6. Sedge-Tufted hairgrass 77

UFC7. Creeping red fescue/Clover $\quad{ }^{\circ}$

UFC8. Kentucky bluegrass-Timothy/Meadow rue $\quad 79$

UFC9. Purple oatgrass-Rough fescue ' $\quad 80$

UFC10. Willow/Kentucky bluegrass 81

UFC11. Sedge-Slender wheatgrass-Rough fescue : : 82

D. Deciduous community types 83

Key to deciduous community types 85

UFD1. Aspen/Rose/Bearberry $\quad 87$

UFD2. Balsam poplar-White spruce/Willow/Yellow Mtn. avens 88

UFD3. Aspen/Rose/Hairy wildrye 89

UFD4. Aspen/Buffaloberry/Hairy wildrye 90

UFD5. Aspen/Marsh reedgrass 91

UFD6. Balsam poplar/Willow/Horsetail 92

E. Conifer community types 93 
UFE1. Lodgepole pine/Bog cranberry 97

UFE2. Lodgepole pine-White spruce/Bunchberry 98

UFE3. Lodgepole pine/Willow/Moss 99

UFE4. Lodgepole pine/Marsh reedgrass 100

UFE5. Black spruce/Willow 101

UFE6. White spruce/Horsetail/Moss $\quad 102$

UFE7. White spruce/Willow 103

UFE8. White spruce/Bearberry 104

UFE9. White spruce/Juniper-Buffaloberry 105

UFE10. White spruce/Moss 106

$\begin{array}{ll}\text { F. Cutblock and burn community types } & 107\end{array}$

• UFF1. Juniper/Hairy wildrye 108

UFF2. Rose/Hairy wildrye 109

UFF2a. Fireweed/Hairy wildrye 110

UFF3. White spruce/Horsetail/Kentucky bluegrass 111.

UFF4. White spruce/Moss (Cutblock) 112

UFF4a. Lodgepole pine-White spruce/Moss 113

UFF5. River alder-Willow/Fireweed-Cow parsnip 114

UFF6. Aspen/Fireweed 115

UFF7. Aspen/Blueberry-Bearberry/Hairy wildrye 116

UFF8. Kentucky bluegrass-Creeping red fescue/Clover 117

$\begin{array}{ll}\text { Literature cited } & 118\end{array}$

\section{List of Figures}

Figure 1. Overview of native shrub and grassland complex in the Upper Foothills subregion

Figure 2. Typical native shrub and grassland transition zone in the Upper Foothills subregion

Figure 3. Layout of plant community types for native grass and shrublands in the landscape of the Upper Foothills subregion

Figure 4 . Heavily grazed Kentucky bluegrass/Dandelion community type

Figure 5. Aspen/Marsh reedgrass community type on south facing slopes in the Upper Foothills subregion

Figure 6. The Lodgepole pine-White spruce/Bunchberry community is the dominant conifer community type in the Upper Foothills 
subregion

93

Figure 7. The Juniper/Hairy wildrye community type results from the harvesting of a White spruce/Juniper community

\section{List of Tables}

Table 1. Ecosites and ecosite phases of the Upper Foothills subregion 6 c5 yellow mountain avens

c6 hairy wildrye sedge

15

c2 hairy wildrye Aw harvested

c4 hairy wildrye Sw harvested

elb tall bilberry/arnica P1 harvested

e1b tall bilberry/arnica Sw harvested

ff fescue-california oatgrass ecosite

ffl grassland

ff2 shrubland

ffl grazed grassland

23

ff2 grazed shrubland

f4 bracted honeysuckle harvested

g2 forb meadow grazed

26

g3 grass meadow

27

g3 grass meadow grazed

28

$\mathrm{j} 2$ horsetail $\mathrm{Pb}$

29

j1 horsetail Sw harvested

30

Table 2. Native grass and shrublands of the Upper Foothills subregion

Table 3 . Shrublands and grasslands modified by heavy grazing in the

Upper Foothills subregion

Table 4. Deciduous community types within the Upper Foothills subregion

Table 5. Conifer community types of the Upper Foothills subregion

\section{List of Maps}

Map 1. Location of the Upper Foothills subregion in Alberta 


\begin{abstract}
The Upper Foothills subregion is found elevationally below the Subalpine and above the Lower Foothills subregions. It is dominated by closed canopied lodgepole pine forests. In the valley bottoms the shrub and grassland community types are a classic example of multiple use land, providing summer range for livestock, prime habitat for many species of wildlife, productive watersheds, and recreational areas. Despite the importance of these vegetation types for livestock grazing, there is little information available on how grazing affects their production. There is little information on forage productivity, carrying capacity and the associated community types with grazing. The lack of information makes it very difficult to development management prescriptions for multiple use. As a result a "Carrying capacity guide" was developed for the Upper Foothills subregion to provide a framework that would easily group the vegetative community types. It is hoped this classification system can be used by field staff to assess carrying capacity and evaluate range condition on lands within the region.

- This guide represents the analysis of 470 plots described in the Upper Foothills subregion, near Grande Cache (Willmore Wilderness Park) and west of Rocky Mtn. House during the summers of 1990-1999. The 470 plots represent 67 community types. These types are split into:
\end{abstract}
A. Native grasslands
18 community types
B. Native shrublands
12 community types
C. Grazing modified types
11 community types
D. Deciduous types
6 community types
E. Conifer types
10 community types
F. Cutblocks and burns
10 community types

The dominant plant species, canopy cover, environmental conditions, response to grazing, forage production and carrying capacity are outlined for each type. 
Digitized by the Internet Archive in 2016 


\section{Introduction}

The province of Alberta is covered by a broad spectrum of vegetation regions from prairie in the South, to alpine vegetation in the mountains and dense forests in the Central and Northern parts of the province. These broad vegetation regions have been classified into 6 natural regions and 20 subregions for the province (Dept. of Environmental Protection, 1994). Each of the regions consists of groups of plant communities which are influenced by environmental conditions and human impacts. Intensive management of these regions requires the ability to recoqnize the vegetative communities that have similar productivities and respond to disturbance in the same way. The increase in use of Alberta's northern forests has recently stimulated efforts to develop detailed classification systems. Some of these classification systems include Field guide to Forest ecosystems of West Central Alberta (Corns and Annas, 1986) and Field Guide to Ecosites of West-Central Alberta (Beckingham et al., 1996).

The vegetative communities in the province of Alberta are highly regarded by most resource managers for their ability to provide a wide variety of benefits. They are a classic example of multiple use land, providing summer range for livestock, prime habitat for many species of wildlife, productive watersheds and recreational areas. Despite the importance of these vegetation types for livestock grazing, there is little information available on how grazing affects their production. Specifically, there is little data on the levels of utilization which are detrimental to communities growth. There is also no data on forage productivity, carrying capacity and associated community types with grazing. Traditionally, these community types have been rated at $5 \mathrm{ac} / \mathrm{AUM}$ or $60 \mathrm{ac} / \mathrm{head} / \mathrm{year}$, but recent work has shown that productivity can vary significantly depending upon the ecological conditions of the site.

The purpose of this guide was to develop a framework that would easily group the vegetative community types in the Upper Foothills subregion of the province. The ultimate goal is a classification system that can be used by the field staff to assess carrying capacity and evaluate range condition on lands within the region. This guide supplements the work done by Beckingham et al. (1996) on the forested community types in the Upper foothills subregion. Their guide describes 65 community types on 13 ecosites. Beckingham's guide is a good description of the forested community types found within the subregion, but it does not include forage production values and carrying capacities. It also only provides a brief description of the native shrubland and grassland communities which are extensively utilized by livestock and wildlife in this subregion.

\section{Climate of the Upper Foothills subregion}

This subregion is found elevationally below the subalpine and above the Lower Foothills subregions. It ranges in elevation from 1200-1500m at lower latitudes and from 1000-1250 $\mathrm{m}$ at higher latitudes. It is dominated by closed canopy lodgepole pine forests with the potential climax species on reference sites being white spruce and black spruce. This 
subregion can be distinguished from the Subalpine subregion by the lack of engelmann spruce and from the Lower Foothills by the lack of aspen.

This subregion has a boreal climate which is modified by the Rocky Mountains. The average annual precipitation is $538 \mathrm{~mm}$ with over half the precipitation recieved in the summer months $(340 \mathrm{~mm})$. The temperature averages $11.5^{\circ} \mathrm{C}$ in the summer and $-6.0^{\circ} \mathrm{C}$ in the winter. These temperatures are milder and not nearly as extreme as the other subregions within the Boreal forest and Foothills natural regions.

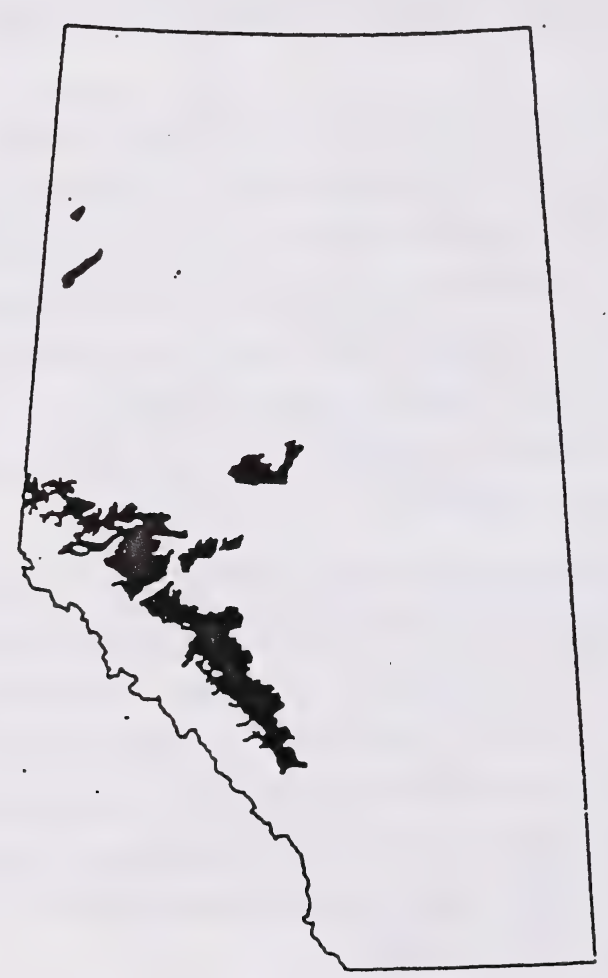

Map 1. Location of Upper Foothills subregion in Alberta 


\section{Methods}

A community type approach (Mueggler, 1988) to classification was chosen in preference to the habitat type approach (Daubenmire, 1952) or ecosystem association approach (Corns and Annas, 1986) because of the lack of understanding of the successional sequences of the communities. Community types are aggregates of similar plant communities based upon existing floristics regardless of successional status (Mueggler, 1988). Community types are what is actually seen in the field. After defining the community types, they then can be linked to the ecosystem associations developed by Corns and Annas (1986) and Beckingham (1994). In the mean time community types can be used as the basis for mapping and range management planning.

Initially, grazing dispositions were inventoried by forest region in order to develop management plans following the procedure outlined in the Rangeland Resource Information System (1991). Individual plots were initially classified within a forest region using cluster analysis (SAS) and ordination (DECORANA, Gauch, 1982). These types were described in -individual carrying capacity guides for each forest. This led to differences in classification of the same types between forests, particularly for deciduous forest types. In an effort to standardize the community name and gain some understanding of each community types ecology, all plots sampled in each forest were reclassified. As the study progressed it became quite evident that there were differences in the productivity of the communities between ecoregions. As a result, it was decided to develop the classification within the ecoregion framework (Strong and Leggat, 1992). An ecoregion is a geographical area that has broad vegetation zones combined with climatic data (Strong and Anderson, 1980). As a result, the vegetation within each ecoregion is strongly influenced by the climatic conditions. Recently, the department has adapted the Natural and Subregions of Alberta classification system. This system incorporates the Natural regions and subregions classification used by Parks with the Ecoregions of Alberta classification used by Forestry, Lands and Wildlife. The Upper Foothills subregion and Upper Boreal Cordilleran ecoregion share the same boundary.

Sampling for this guide occurred within the Upper Foothills subregion. This guide outlines the classification of 439 plots described during the summers of 1990-2000 in the Southern and Northern East Slope Forest regions.

A plot consisted of a $10 \times 10 \mathrm{~m}$ macroplot and ten randomly selected $1 \times 1 \mathrm{~m}$ microplots to record the canopy cover of shrubs and ten nested $20 \times 50 \mathrm{~cm}$ microplots to record the canopy cover of forbs and grass. The inventory followed the Range Survey Manual (1992) and uses the MF5 form. At each macroplot a 50x100 cm was clipped and separated into trees, shrubs, forbs and graminoids, oven dried and weighed. The recommended stocking rate is based on 25 percent of the total production for forested types and 50\% total production for grass and shrubland types and the fact that one animal requires $455 \mathrm{~kg}$ of dry weight material for one month of grazing. 


\section{How to use the guide}

First decide what category the community type is in. If it is in the Native grass and Shrub category it will not have tree cover and be found on steep south facing slopes or moist lowland areas adjacent to streams and rivers. The predominant species will be native grasses, willow and bog birch. The Grazing modified community types will resemble the native shrub and grassland community types, but will show signs of extensive grazing pressure. These community types will be dominated by grazing resistant species Kentucky bluegrass, clover and dandelion. A couple of moderately grazed community types with a predominant native species cover are also found in this category.

The Deciduous category will be plant communities dominated by deciduous tree species aspen and balsam poplar and the Conifer category will be plant communities dominated by white spruce, lodgepole pine or black spruce tree species.

- In order to understand how the community types in this guide are related to the ecosites and ecosite phases outlined in "Ecosites of West-Central Alberta" (Beckingham et al., 1996), the community types in this guide are arranged by ecosite and ecosite phase (Table 1). Ecosites are defined as ecological units that develop under similar environmental influences (climate, moisture and nutrient regime). An ecosite phase is a subdivision of the ecosite based on the dominant species in the canopy. Table 1 is a reproduction of Figure 14 in the Ecosites of West-Central Alberta guide with the community types in this guide highlighted. For the most part the ecosites and ecosite phases are the same, particularly for the forested community types, but a number of new ecosites and ecosite phases had to be created for the grass and shrubland community types (Table 1 ). These included (ff)(mesic/rich) fescuecalifornia oatgrass ecosite, and the (c5) yellow mtn avens, (c6) hairy wildrye grassland, (ff1)grassland, (ff2) shrubland, ( $\mathrm{g} 3$ ) grass meadow and $(\mathrm{j} 2$ ) horsetail $\mathrm{Pb}$ ecosite phases. The "Grazing succession" and "Harvesting succession" categories (Table 1) outline the successional sequence the community types will undergo with increased grazing pressure or harvesting. A number of new ecosite phases were created for these categories. These included (c2) harvested Aw, (c4) harvested Sw, (e1) tall bilberry/arnica Pl-Sw harvested, (ff1) grazed grassland, (ff2) grazed shrubland, (f4)bracted honeysuckle Sw harvested, (g2) grazed forb meadow, (g3) grazed grass meadow and (j1) horsetail Sw harvested. All of the new ecosites and ecosite phases are summarized within this guide. 


\section{Results}

The analysis of the 470 plots distinguished 67 community types. These types were split into 6 categories:
A.) Native grasslands
(18 types)
B.) Native shrublands
(12 types)
C.) Grazing modified types
(11 types)
D.) Deciduous types
(6 types)
E.) Conifer types
(10 types)
F.) Cutblock types
(10 types)

The dominant plant species, canopy cover, environmental conditions, forage production and carrying capacity are outlined for each community type. 


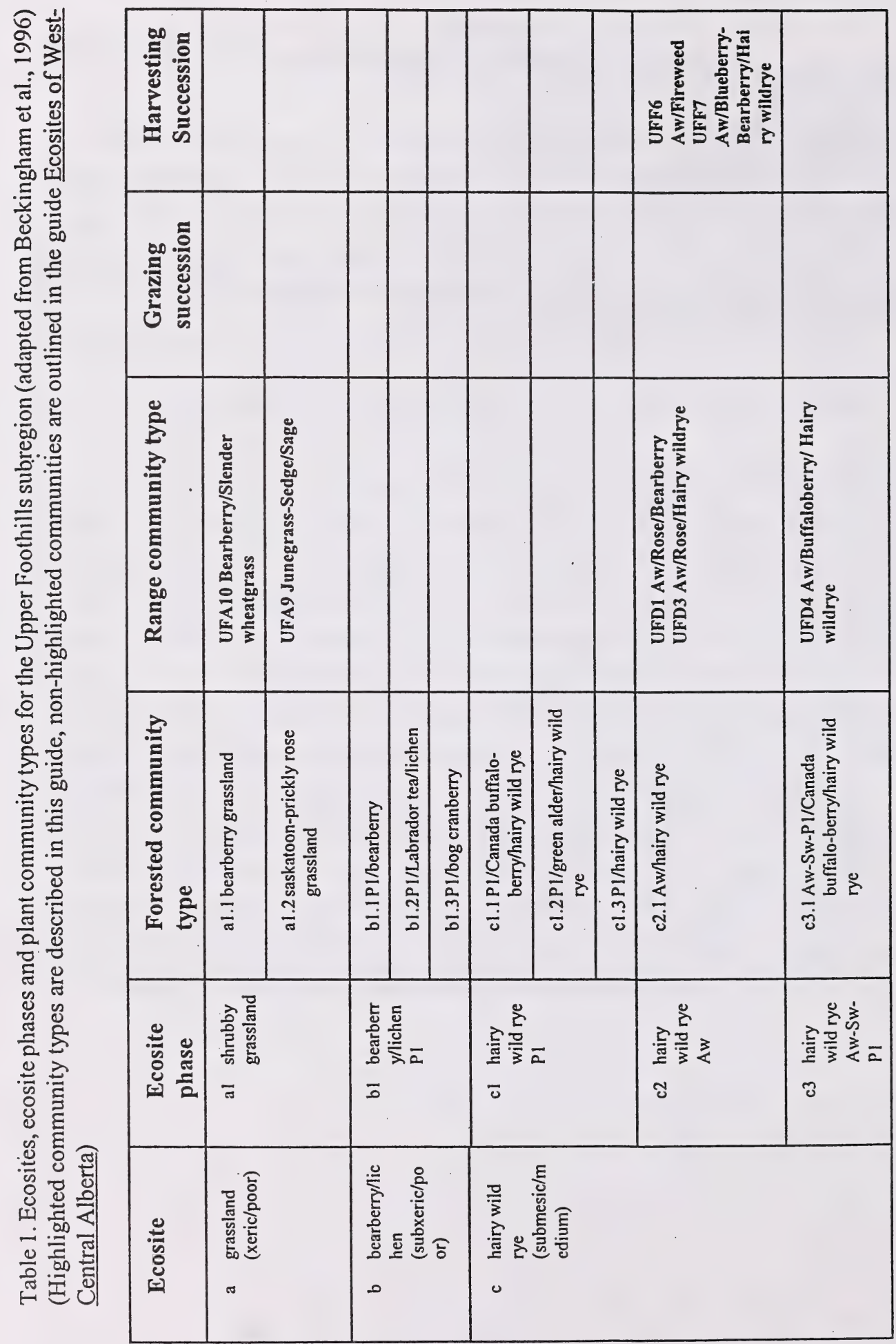




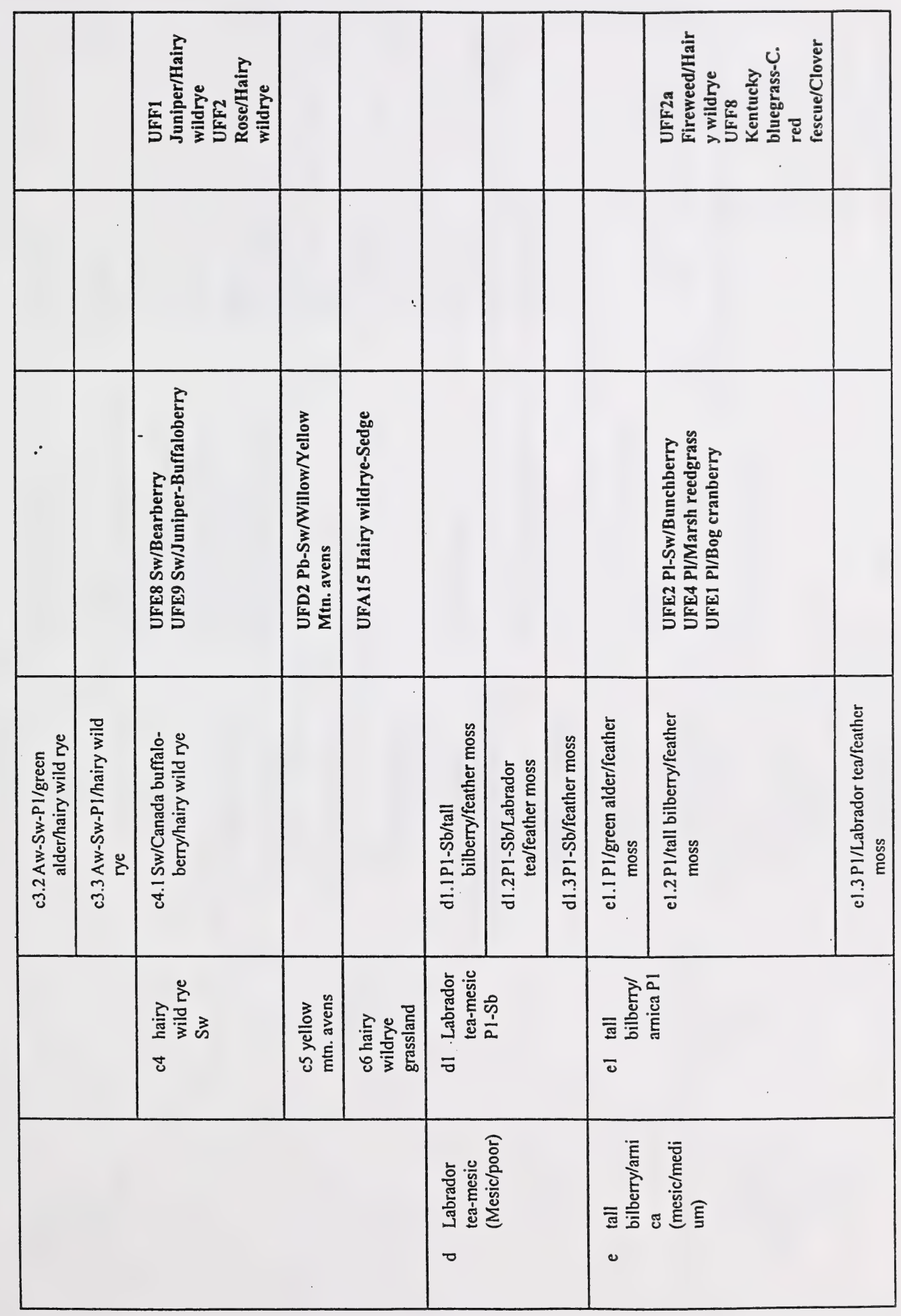




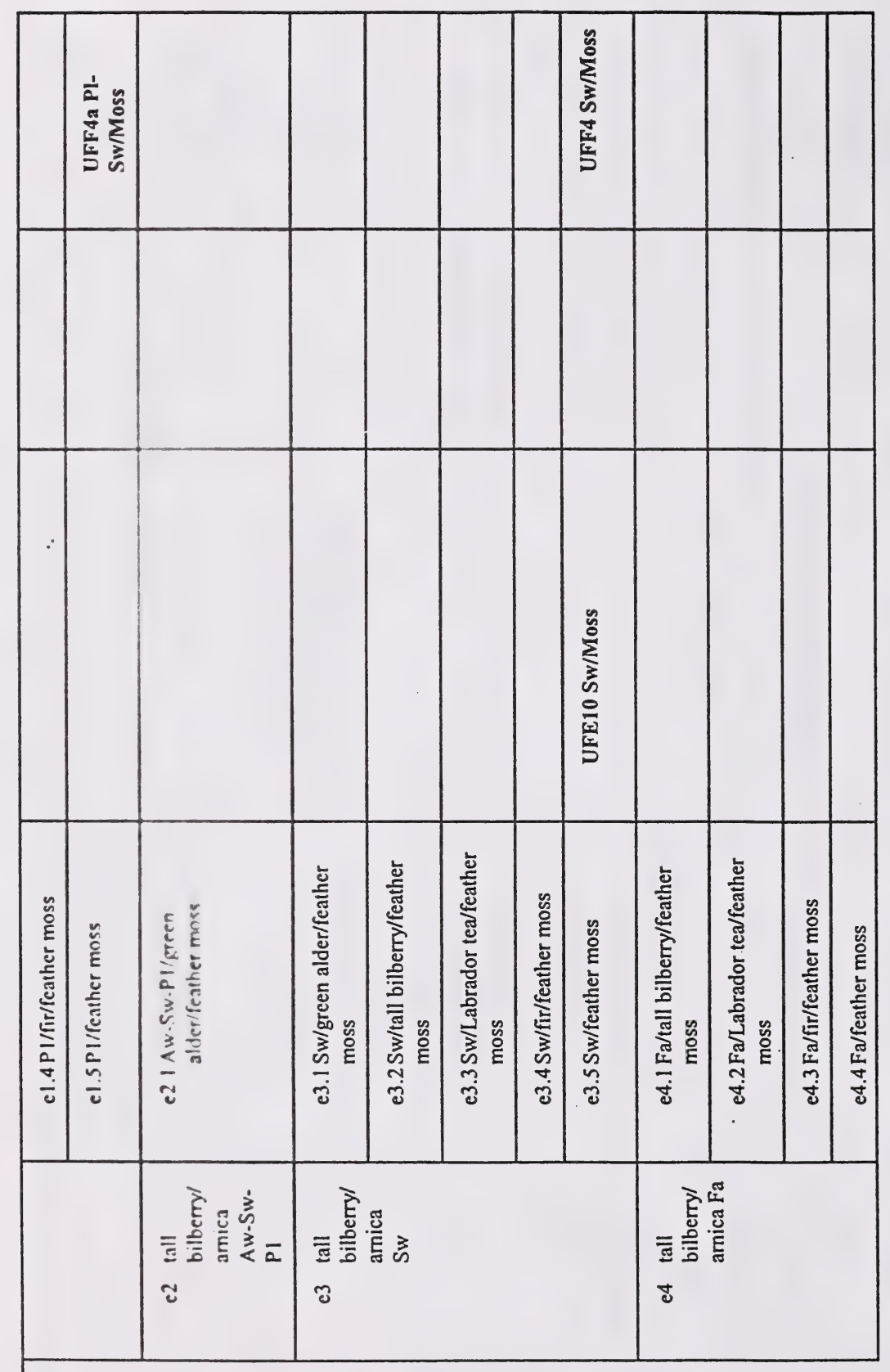

$\infty$ 


\begin{tabular}{|c|c|c|c|c|c|}
\hline 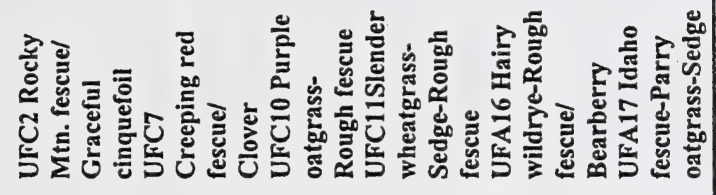 & 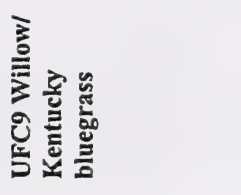 & & & & \\
\hline 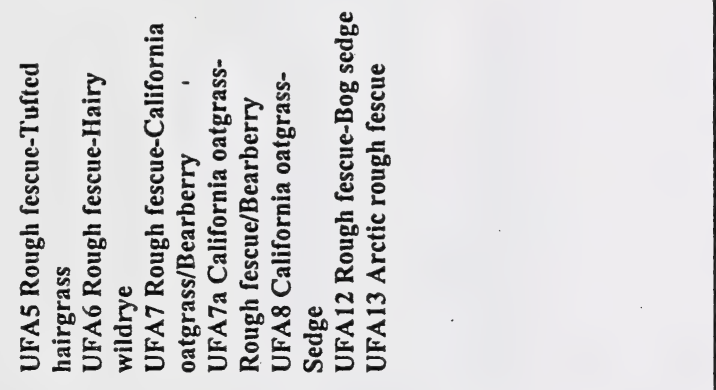 & 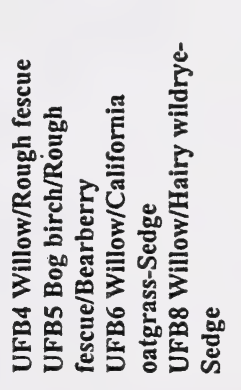 & & 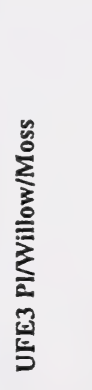 & & \\
\hline & & 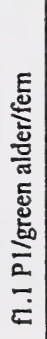 & 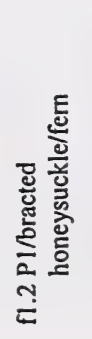 & 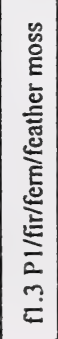 & 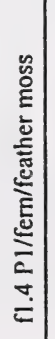 \\
\hline 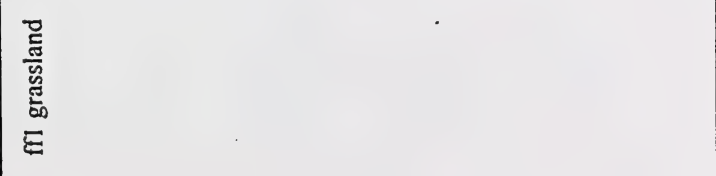 & 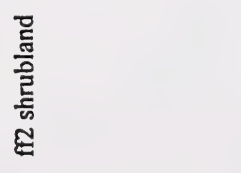 & \multicolumn{4}{|c|}{ 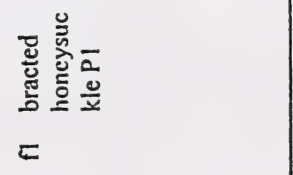 } \\
\hline 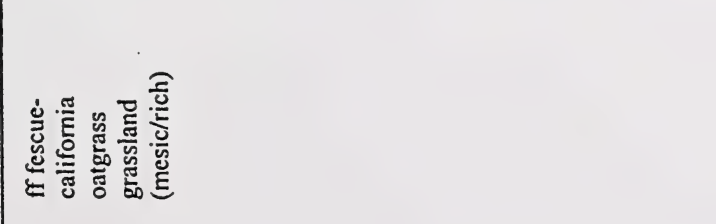 & & & 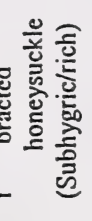 & & \\
\hline
\end{tabular}




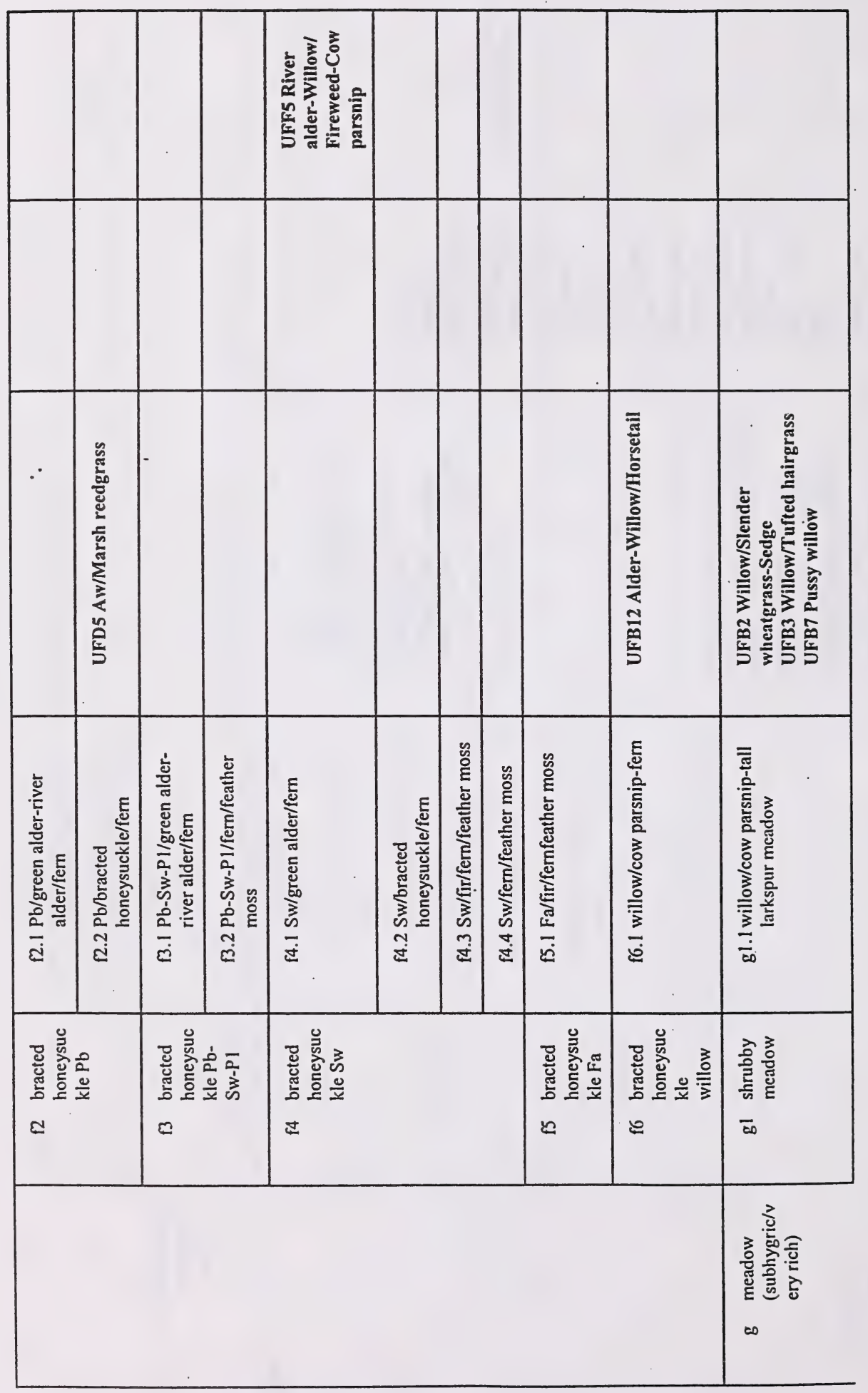




\begin{tabular}{|c|c|c|c|c|c|}
\hline & & 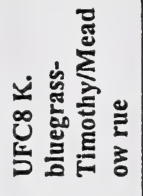 & 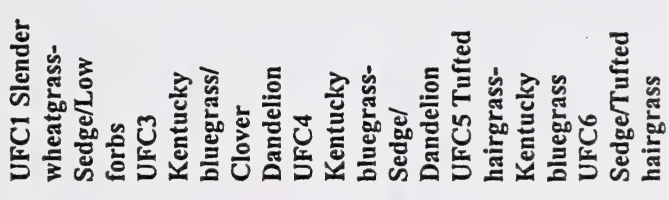 & & \\
\hline 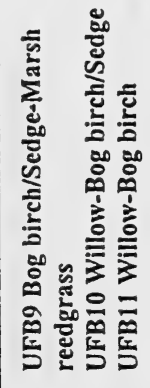 & & 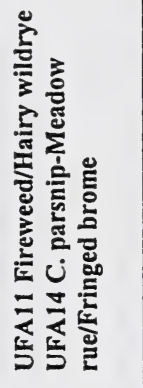 & 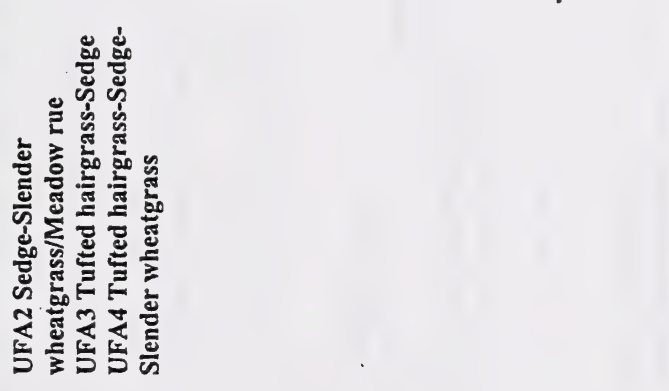 & & \\
\hline 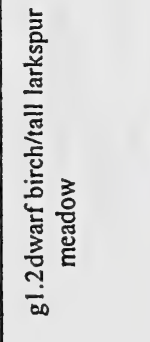 & 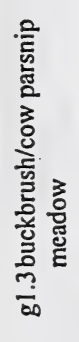 & 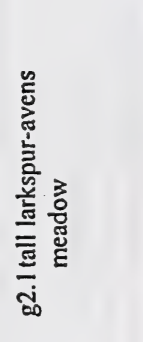 & & 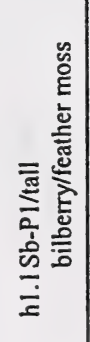 & 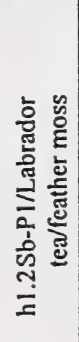 \\
\hline & & 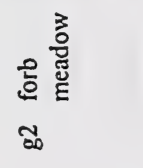 & 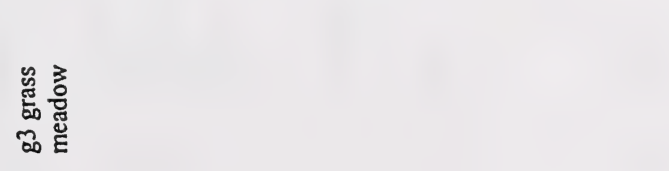 & \multicolumn{2}{|c|}{ 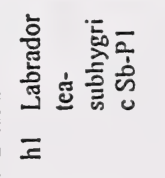 } \\
\hline & & & & 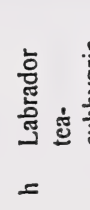 & 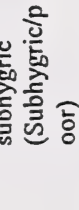 \\
\hline
\end{tabular}




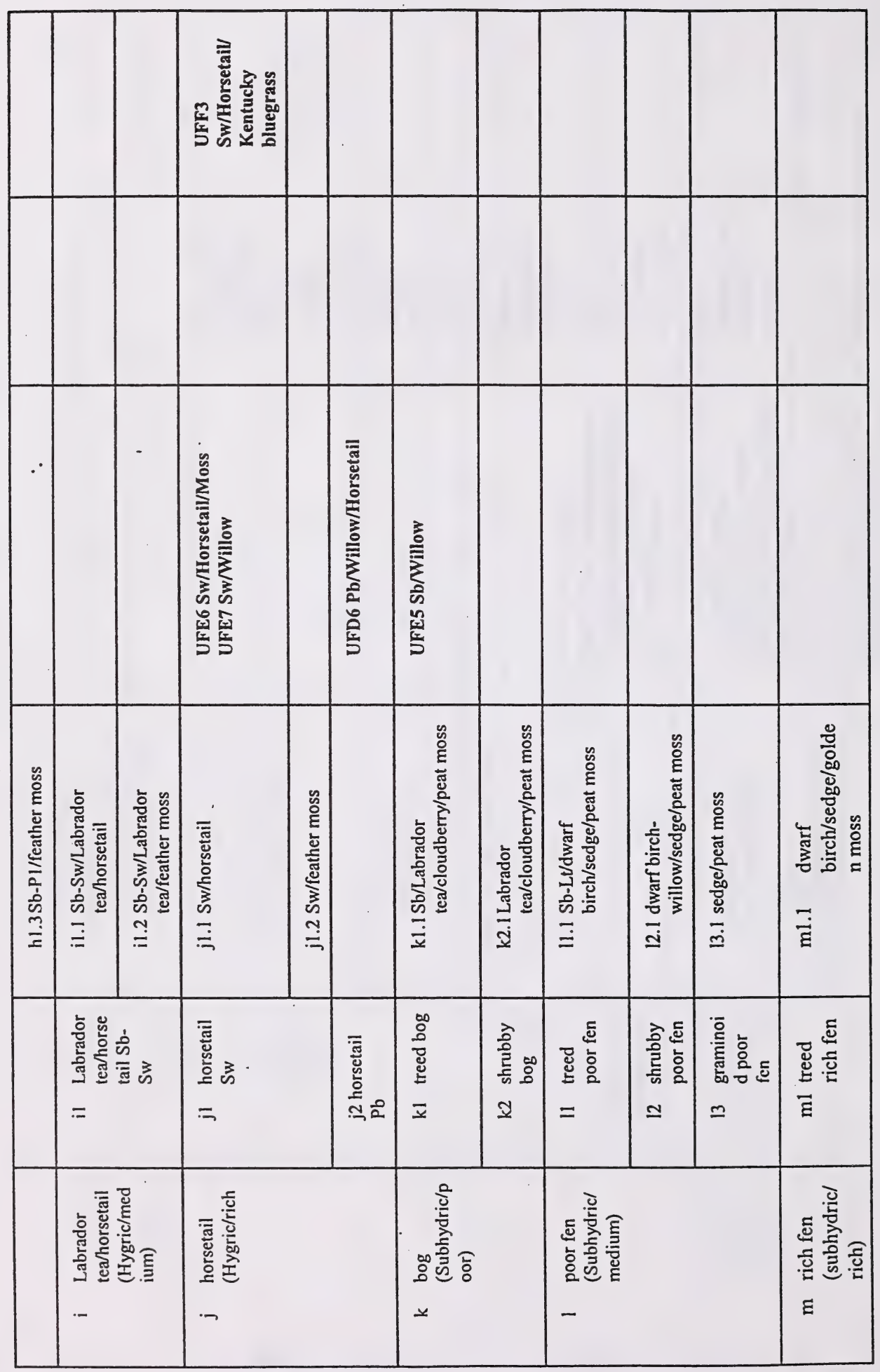




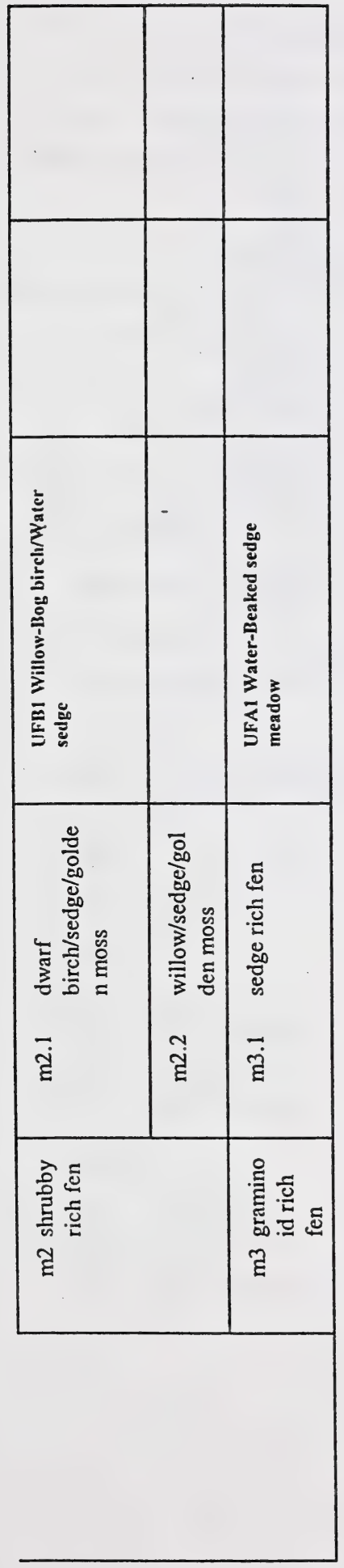

2 
c5 Yellow mountain avens $(n=1)$

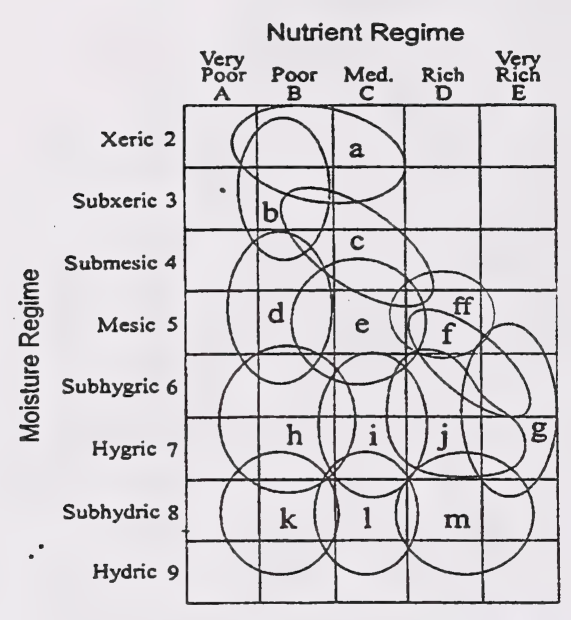

\section{CHARACTERISTIC SPECIES}

Tree

[7] Balsam poplar

[5] White spruce

Shrub

[16] Yellow mountain avens

[13] Willow

[9] Buffaloberry

[3] Bearberry

Forb

[11] Alpine hedysarum

[11] Scouring rush

[4] Alpine milk vetch

\section{Grasses}

[2] Blunt Sedge

\section{SITE CHARACTERISTICS}

Moisture regime: mesic, submesic

Nutrient regime: medium, poor

Topographic position: crest, upper slope, midslope

Slope: level

Aspect: northerly, level

\section{SOIL CHARACTERISTICS}

Organic thickness: (6-15), (0-5)

Humus form: mor, moder

Surface texture: SiL, L, CL, LS, C

Effective texture: SCL, CL, SiL, SL, L, C

Depth to Mottles/Gley: none

Drainage: well, moderately well, rapidly

Parent material: $M, M / R, G F$

Soil subgroup: BR.GL, O.EB, E.EB, O.GL

RANGE PLANT COMMUNITY TYPES

UFD2. $\mathrm{Pb}-\mathrm{Sw} / \mathrm{Willow} /$ Yellow Mtn. avens $\mathrm{n}=1$ 


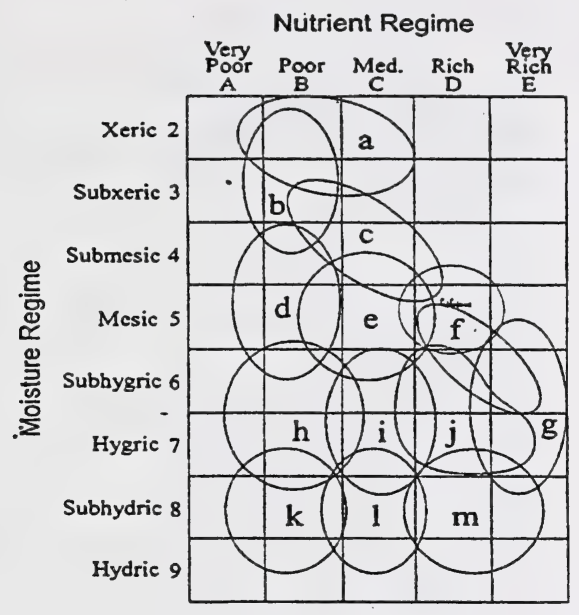

\section{SITE CHARACTERISTICS}

Moisture regime: mesic, submesic

Nutrient regime: medium, poor

Topographic position: crest, upper slope, midslope

Slope: level

Aspect: northerly, level

\section{SOIL CHARACTERISTICS}

Organic thickness: $(6-15),(0-5)$

Humus form: mor, moder

Surface texture: SiL, L, CL, LS, C

Effective texture: SCL, CL, SiL, SL, L, C

Depth to Mottles: Gley: none

Drainage: well, moderately well, rapidly

Parent material: $M, M / R, G F$

Soil subgroup: BR.GL, O.EB, E.EB, O.GL

\section{RANGE PLANT COMMUNITY TYPES}

UFA15. Hairy wildrye-Sedge $n=1$

\section{CHARACTERISTIC SPECIES}

\section{Shrub}

\section{[4] Bearberry}

Forb
[5] Fireweed
[9] Showy locoweed
[4] American vetch
[8] Strawberry
[2]. Graceful cinquefoil

\section{Grasses}
[31] Hairy wildrye
[3] Arctic bluegrass
[3] Sedge
[1] Slender wheatgrass 


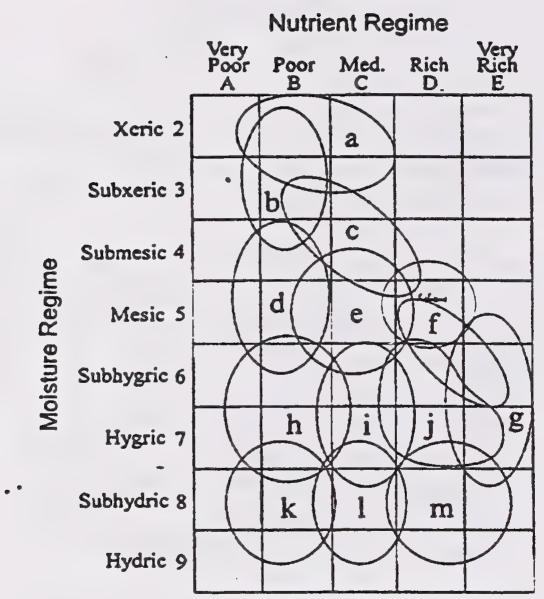

\section{SITE CHARACTERISTICS}

Moisture regime: mesic, submesic

Nutrient regime: medium, rich

Topographic position: upper slope, midslope, level

Slope: $(3-5) \%$

Aspect: north, east

\section{SOIL CHARACTERISTICS}

Organic thickness: (6-15), $(0-5)$

Humus form: mor

Surface texture: SiL, L, CL, LS, SiCL, C

Effective texture: SCL, SiCL, CL, SiC, SL, C

Depth to Mottles/Gley: none

Drainage: well, moderately well

Parent material: $M, C, X$

Soil subgroup: BR.GL, O.MB, O.GL, O.EB, O.DYB,

GL.GL, E.EB, D.GL

\section{RANGE PLANT COMMUNITY TYPES}

UFF6. Aw/fireweed $n=1$

UFF7. Aw/Blueberry-Bearberry/Hairy wildrye $n=2$

\section{CHARACTERISTIC SPECIES}

\section{Trees}
[1] White spruce
[ 7 ] Aspen
[ 1 ] Lodgepole pine

Shrub

[ 7 ] Blueberry

[2] Bog cranberry

[4] Prickly rose

[2] Green alder

Forb

[18] Fireweed

[6] Horsetail

[ 3 ] Heart leaved arnica

\section{Grasses}

[ 4 ] Marsh reedgrass

[1] Sedge 


\section{c4b Harvested hairy wildrye/ Sw} $(n=14)$

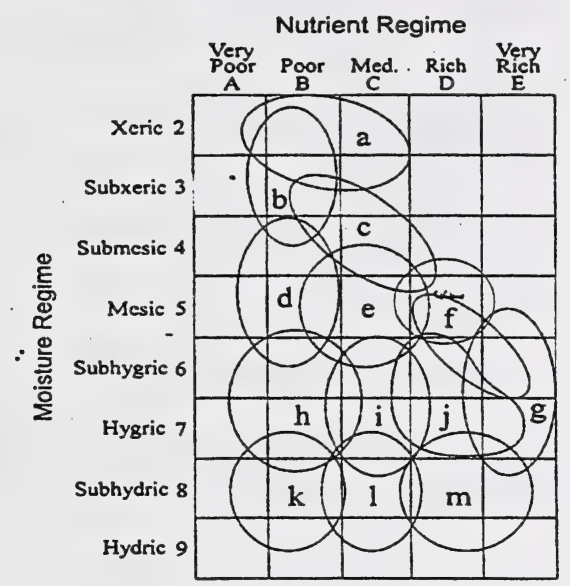

\section{CHARACTERISTIC SPECIES}

Tree

[10] White spruce

[7] Balsam poplar

[3] Aspen

Understory tree
[2] White spruce
[2] Balsam poplar
[1] Aspen

Shrub
[3] Shrubby cinquefoil
[5] Rose
[7] Creeping juniper
[8] Willow
[3] Bearberry

Forb
[3] Showy locoweed
[3] Northern hedysarum
[5] Northern bedstraw

\section{Grasses}

[ 3 ] Sedge

[21] Hairy wildrye

[ 3 ] Slender wheatgrass

\section{SITE CHARACTERISTICS}

Moisture regime: mesic, submesic

Nutrient regime: medium, poor

Topographic position: upper slope, midslope, level Slope: $(2-10) \%$

Aspect: southeasterly, southwesterly, south

\section{SOIL CHARACTERISTICS}

Organic thickness: (6-15), (0-5)

Humus form: mor

Surface texture: SiL, L, CL, LS, SiCL, C

Effective texture: $\mathrm{SCL}, \mathrm{SiCL}, \mathrm{CL}, \mathrm{SiC}, \mathrm{SL}, \mathrm{C}$

Depth to Mottles/Gley: none

Drainage: well, moderately well

Parent material: $\mathrm{M} / \mathrm{R}, \mathrm{FL}, \mathrm{C} / \mathrm{M}$

Soil subgroup: O.EB, E.EB, BR.GL

\section{RANGE PLANT COMMUNITY TYPES}

UFF1. Juniper/Hairy wildrye $n=4$

UFF2. Rose/Hairy wildrye $n=10$ 


\section{e1b Harvested PV/ Tall bilberry/ Feather moss $(n=27)$}

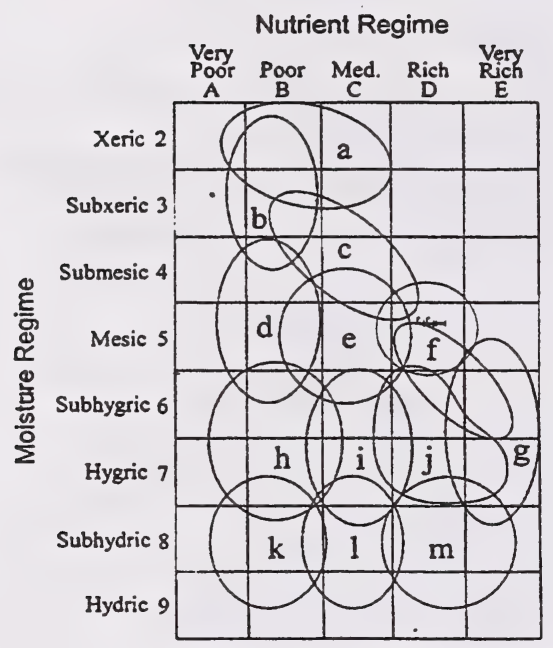

\section{CHARACTERISTIC SPECIES}

Shrub

[1] Rose

Forb

[4] Fireweed

[3] Clover

\section{Grasses}
[2] Creeping red fescue
[3] Kentucky bluegrass
[8] Hairy wildrye
[2] Timothy

\section{SITE CHARACTERISTICS}

Moisture regime: mesic to subxeric

Nutrient regime: medium, poor

Topographic position: midslope, level, upper slope Slope: $(2-41) \%$

Aspect: variable

\section{SOIL CHARACTERISTICS}

Organic thickness: $(0-4)$

Humus form: moder

Surface texture: SiL

Effective texture: $\mathrm{SiL}$

Depth to Mottles/Gley: none

Drainage: well, moderately well

Parent material: E, GF

Soil subgroup: O.EB

\section{RANGE PLANT COMMUNITY TYPES}

UFF2a. Fireweed/Hairy wildrye $n=22$

UFF8. Kentucky bluegrass-C. red fescue/Clover $n=5$ 


\section{e3b Harvested tall bilberry/ Arnica/ Sw ( $\mathrm{n}=6)$}

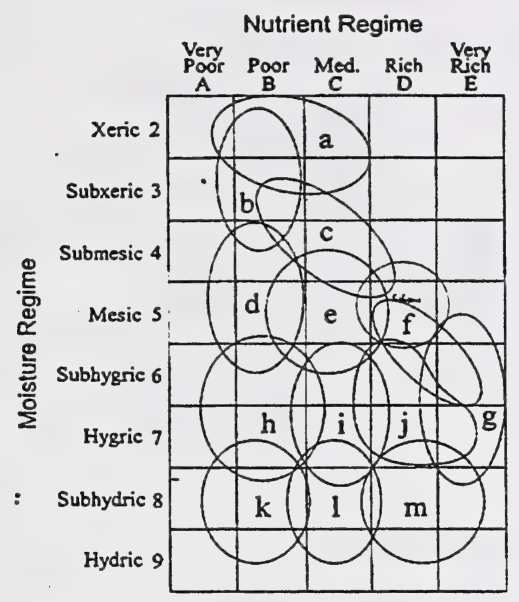

\section{SITE CHARACTERISTICS}

Moisture regime: mesic to subxeric

Nutrient regime: medium, poor

Topographic position: midslope, level, upper slope Slope: $(2-41) \%$

Aspect: variable

\section{SOIL CHARACTERISTICS}

Organic thickness: $(0-4)$

Humus form: moder,

Surface texture: SiL

Effective texture: $\mathrm{SiL}$

Depth to Mottles/Gley: none

Drainage: well, moderately well

Parent material: E, GF

Soil subgroup: $O, E B$

\section{RANGE PLANT COMMUNITY TYPES}

UFF4a. Pl-Sw/Moss $n=6$

\section{CHARACTERISTIC SPECIES}

\section{Tree}

$\begin{array}{ll}{[3]} & \text { White spruce } \\ {[10]} & \text { Lodgepole pine }\end{array}$

Shrub

[1] Willow

[2] Bunchberry

Forb
[1] Fireweed
[5] Horsetail

Grasses
[6] Hairy wildrye
[3] Marsh reed grass

Moss
[1] Feather moss
[6] Stair step moss 


\section{GENERAL DESCRIPTION}

This ecosite consists of open grasslands found in valley bottoms, adjacent to rivers and streams, and on south facing slopes. The ecosite tends to be meisc to submesic and occurs on loamy fluvial parent material where flooding and or high water tables increase soil water content and repienish nutrents. The soils on these sites tend to have thick Ah horizons.

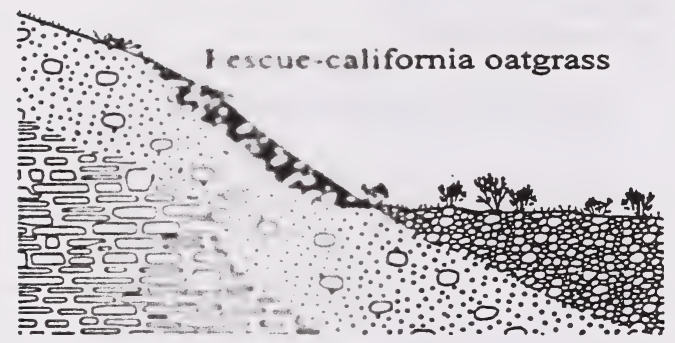

\section{SUCCESSIONAL RELATIONSHIPS}

Due to the nature of the site grasslands often remain the climax

vegetation on these sites. In the moister lower slope positions shrubs often dominate the site with succession to aspen and spruce. Disturbance regime, cold air drainage, and competion from a diverse cover of shrubs, forbs and grasses slow or inhibit the establishment of trees. If trees do become established, the rich loamy soils usually result in rapid growth.

\section{INDICATOR SPECIES}

Rough fescue

California oatgrass

Tufted hairgrass

Sedge

Bearberry

Strawberry

Three flowered avens

Clover

Alpine rough fescue

Shrubby cinquefoil

Slender wheatgrass

Kentucky bluegrass

Hairy wildrye

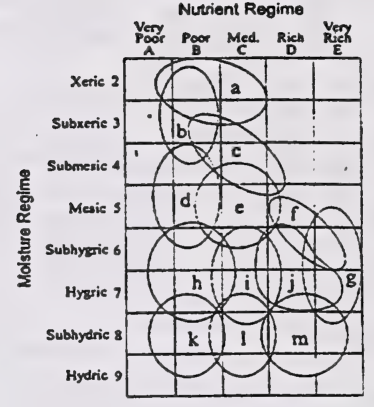

\section{SITE CHARACTERISTICS}

Moisture regime: mesic, submesic

Nutrient regime: rich, medium

Topographic position: crest, upper, mid to lower slope

Slope: $(0-5 \%)(5-20 \%)$

Aspect: south, southwest

\section{SOIL CHARACTERISTICS}

Organic thickness: (0-5)

Humus form: moder, mull

Surface texture: CL, SiL, L

Effective texture: CL, SiL, SL

Depth to Mottles/Gley: none

Drainage: well, moderately well, imperfectly

Parent material: F, C, E, GF

Soil subgroup: O.EB ,O.HR, CU.R

\section{ECOSITE PHASES}

ffl grassland (44)

ff2 shrubland (26) 


\section{ffI Grassland $(n=44)$}

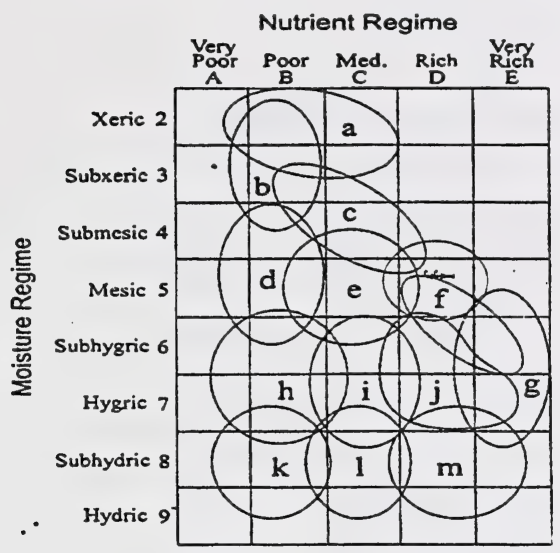

CHARACTERISTIC SPECIES

Shrub

[ 3 ] Bearberry

[ 4 ] Shrubby cinquefoil

Forb

[1]Slender blue beard tongue

[1] Graceful cinquefoil

[9] Three flowered avens

[2] Wild strawberry

\section{Grasses}
[24] Rough fescue
[2] Tufted hairgrass
[4] Hairy wildrye
[9] California oatgrass
[19] Sedge
[6]Slender wheatgrass
[2]Alpine rough fescue

\section{SITE CHARACTERISTICS}

Moisture regime: mesic, submesic

Nutrient regime: rich

Topographic position: midslope, lowerslope, level

Slope: $5-20 \%$

Aspect: southerly

\section{SOIL CHARACTERISTICS}

Organic thickness: (0-5)

Humus form: mull, moder

Surface texture: CL, SiL, L

Effective texture: $\mathrm{CL}$, SiL, SL

Depth to Mottles/Gley: none

Drainage: well, modetately well, imperfectly

Parent material: E, C, F, GF

Soil subgroup: O.HR, O.EB, CU.R

\section{RANGE PLANT COMMUNITY TYPES}

UFA5. Rough fescue-Tufted hairgrass $n=5$

UFA6. Rough fescue-Hairy wildrye $n=11$

UFA7. Rough fescue-California oatgrass/Bearberry $n=4$

UFA7a. Califomia oatgrass-Rough fescue/Bearberry $\mathrm{n}=2$

UFA8. California oatgrass-Sedge $n=10$

UFA12. Rough fescue-Bog sedge $n=9$

UFA13. Arctic rough fescue $n=2$

UFA16. Hairy wildrye-Rough fescue/Bearberry $n=1$ 


\section{ff2 Shrubland $(n=26)$}

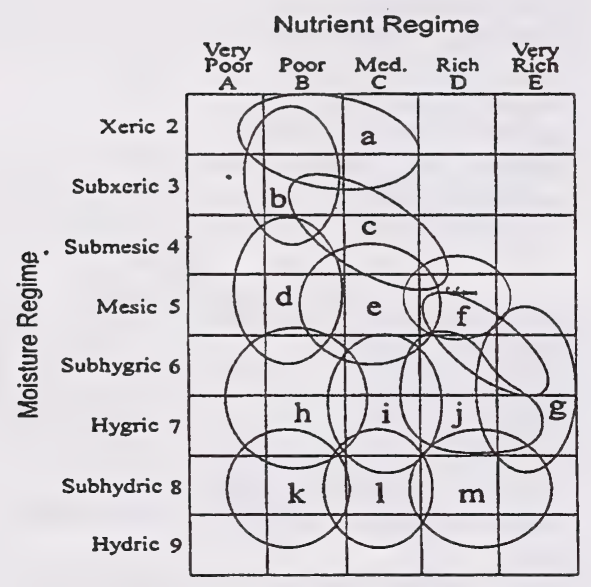

CHARACTERISTIC SPECIES

Shrub

[6] Willow

[17] Bog birch

[4] Bearberry

Forb
[1] Alpine bistort
[5] Strawberry
[1] Lindley's aster
[2] Fireweed

Grasses
[1] Hairy wildrye
[1] Sheep fescue
[4] Sedge
[6] Califomia oatgrass
[12] Rough fescue
[1] Purple oatgrass
[3] Slender wheatgrass

\section{SITE CHARACTERISTICS}

Moisture regime: mesic, subhygric, hygric Nutrient regime: rich

Topográphic position: lowerslope, level Slope: $0-10 \%$

Aspect: south westerly, north easterly

\section{SOIL CHARACTERISTICS}

Organic thickness: $(0-4)$

Humus form: moder

Surface texture: $\mathrm{L}$

Effective texture: $\mathrm{CL}$

Depth to Mottles/Gley: none

Drainage: well, moderately well

Parent material: L, M

Soil subgroup: O.EB

\section{RANGE PLANT COMMUNITY TYPES}

UFB4. Willow/Rough fescue $n=2$

UFB5. Bog birch/Rough fescue/Bearberry $n=18$ UFB6. Willow/Califomia oatgrass-Sedge $n=4$ UFB8. Willow/Hairy wildrye-Sedge $n=2$ 


\section{ffla Grazed grassland $(n=57)$}

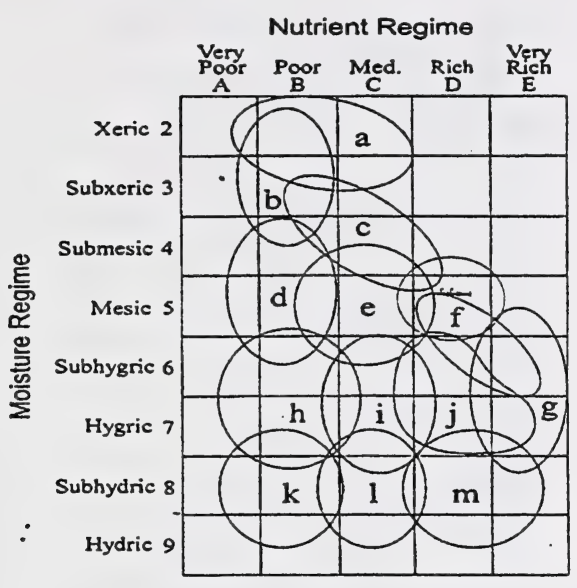

\section{CHARACTERISTIC SPECIES}

\section{Shrub}

[1] Shurbby cinquefoil

[1] Bog birch

Forb

[2] Strawberry

[2] Yarrow

[1] Graceful cinquefoil

[19] Clover

\section{Grasses}

[5] Sedge

[2] Idaho fescue

[2] Parry's oatgrass

[32] Creeping red fescue

[1] Rocky mountain fescue

[15] Kentucky bluegrass

\section{SITE CHARACTERISTICS}

Moisture regime: mesic, submesic

Nutrient regime: rich

Topographic position: midslope, lowerslope, level

Slope: $5-20 \%$

Aspect: southerly

\section{SOIL CHARACTERISTICS}

Organic thickness: (0-5)

Humus form: mull, moder

Surface texture: CL, SiL, L

Effective texture: $C L, S i L, S L$

Depth to Mottles/Gley: none

Drainage: well, moderately well, imperfectly

Parent material: E, C, F, GF

Soil subgroup: O.HR, O.EB, CU.R

\section{RANGE PLANT COMMUNITY TYPES}

UFC2. Rocky Mtn. fescue/Graceful cinquefoil $n=1$ UFC7. Creeping red fescue/Clover $n=24$ UFC10. Purple oatgrass-Rough fescue $n=1$ UFC11. Slender wheatgrass-Sedge-Rough fescue $(n=29)$

UFA17. Idaho fescue-Parry oatgrass-Sedge $n=2$ 


\section{ff2a Grazed shrubland ( $\mathrm{n}=2)$}

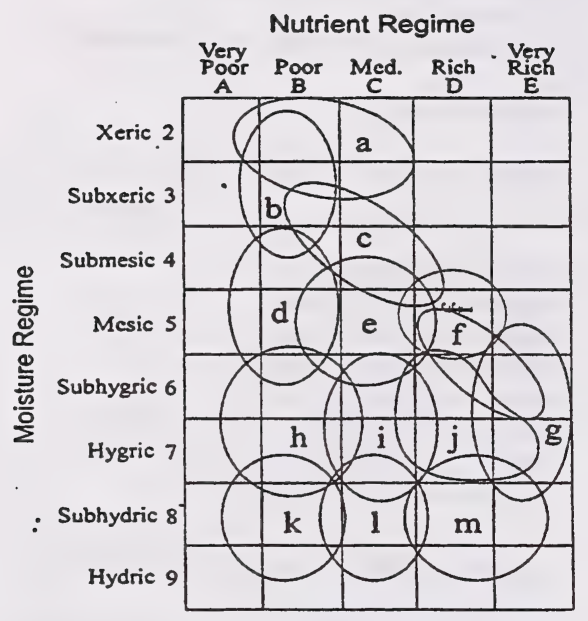

\section{SITE CHARACTERISTICS}

Moisture regime: mesic, subhygric,hygric Nutrient regime: rich

Topographic position: lowerslope, level Slope: $0-10 \%$

Aspect: south westerly, norht easterly

\section{SOIL CHARACTERISTICS}

Organic thickness: $(0-4)$

Humus form: moder

Surface texture: $\mathrm{L}$

Effective texture: $\mathrm{CL}$

Depth to Mottles/Gley: none

Drainage: well, moderately well

Parent material: L, M

Soil subgroup: $\mathrm{O} . \mathrm{EB}$

RANGE PLANT COMMUNITY TYPES

UFC9. Willow/Kentucky bluegrass $n=2$

\section{CHARACTERISTIC SPECIES}

Shrub

[22] Willow

Forb

[11] Dandelion

[7] Yarrow

[2] Strawberry

[5] Tall lungwort

[5] Clover

\section{Grasses}
[12] Kentucky bluegrass
[5] Sheep fescue
[9] Slender wheatgrass
[8] Tufted hairgrass 


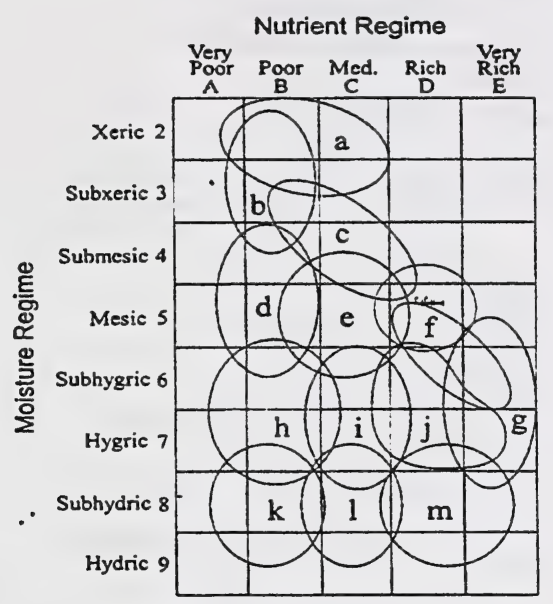

Moisture regime: subhygric, mesic

Nutrient regime: rich

Topographic position: midslope, upper slope, level

Slope: $(20) \%$

Aspect: southeasterly

\section{SOIL CHARACTERISTICS}

Organic thickness: (6-15), (16-25)

Humus form: raw moder

Surface texture: $\mathrm{Si}, \mathrm{L}, \mathrm{SiL}, \mathrm{SiCL}, \mathrm{SiC}$

Effective texture: C, SiCL, SiC, Si, L, CL

Depth to Mottles/Gley: none

Drainage: Moderately well, imperfectly

Parent material: $M, L, E, C / M$

Soil subgroup: D.GL, O.GL, E.EB, E.DYB

\section{RANGE PLANT COMMUNITY TYPES}

UFF5. River alder-Willow/Fireweed-Cow parsnip $n=1$

\section{CHARACTERISTIC SPECIES}

Tree

[3] White spruce

[5] Aspen

Shrub

[5] Willow

[5] River alder

Forb

[21] Fireweed

[13] Cow parsnip

[10] Stinging nettle

[10] White geranium

[8] Tall lungwort

[6] Horsetail

\section{Grasses}

[4] Slender wheatgrass

[3] Marsh reedgrass 


\section{g2a Grazed forb meadow $(\mathrm{n}=4)$}

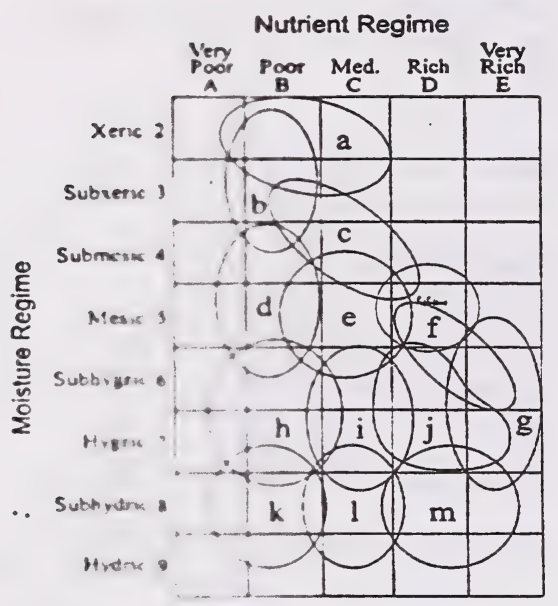

\section{SITE CHARACTERISTICS}

Moisture regime: submesic, subhygric Nutrient regime: medium, rich

Topographic position: midslope, level Slope: $(0-6) \%$

Aspect: southeast, southwest

\section{SOIL CHARACTERISTICS}

Organic thickness: (6-15)

Humus form: mull

Surface texture: $\mathrm{SiC}, \mathrm{SiL}, \mathrm{C}$

Effective texture: $\mathrm{SiC}, \mathrm{C}$

Depth to Mottles/Gley: none

Drainage: moderately well, imperfectly

Parent material: L, F

Soil subgroup: R.G, O.R

\section{RANGE PLANT COMMUNITY TYPES}

UFC8. K. Bluegrass-Timothy/Meadow rue $(n=4)$

\section{CHARACTERISTIC SPECIES}

Shrub

\section{[2] Wrilow}

Forb

$\begin{array}{ll}{[13]} & \text { Dandelion } \\ {[7]} & \text { Cow parsnip } \\ {[5]} & \text { Clover } \\ {[7]} & \text { Veiny meadow rue }\end{array}$

\section{Grasses}
[7] Slender wheatgrass
[2] Smooth brome
[17] Timothy
[22] Kentucky bluegrass 
g3 Grass meadow $(n=46)$

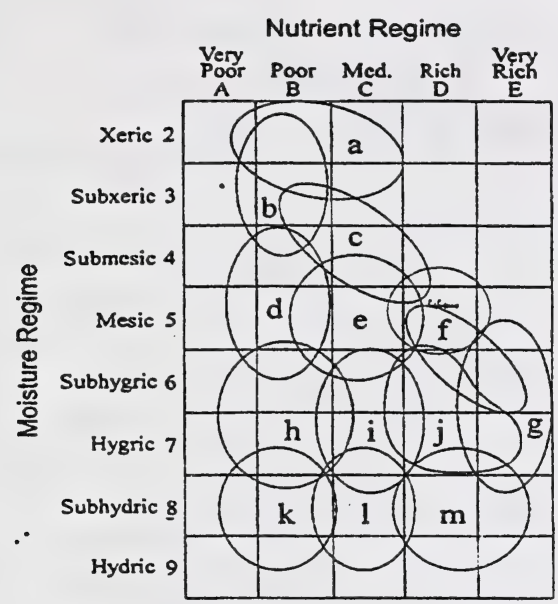

\section{CHARACTERISTIC SPECIES}

Shrub

[1] Barclay's willow

[2] Willow

[2] Bog birch

Forb

[7] Lindley's aster

[8] Yarrow

[8] Veiny meadow rue

[5] Graceful cinquefoil

[6] Wild strawberry

\section{Grasses}
[36] Sedge species
[3] Slender wheatgrass
[25] Tufted hairgrass

Moisture regime: mesic, subhygric,hygric

Nutrient regime: very rich

Topographic position: lowerslope, level

Slope: $0 \%, 5-40 \%$

Aspect: south westerly, south easterly, south and east

\section{SOIL CHARACTERISTICS}

Organic thickness: $(0-5)$

Humus form: moder

Surface texture: SiL, L

Effective texture: SiL, LS, L

Depth to Mottles/Gley: none

Drainage: well, moderately well

Parent material: GL, GF, F

Soil subgroup: O.DYB, O.HR

\section{RANGE PLANT COMMUNITY TYPES}

UFA2. Sedge-Slender wheatgrass/Meadow rue $n=3$ UFA3. Tufted hairgrass-Sedge $n=35$

UFA4. Tufted hairgrass-Sedge-Slender wheatgrass $n=8$ 


\section{g3a Grazed grass meadow $(\mathrm{n}=74)$}

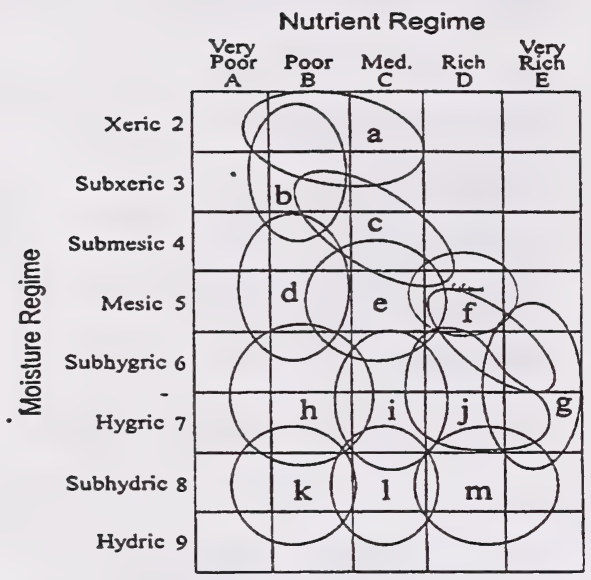

CHARACTERISTIC SPECIES

Shrub

[1] Willow

Forb

[4] Strawberry

[8] Yarrow

[9] Graceful cinquefoil

[15] Dandelion

[5] Clover

[7] Veiny meadow rue

\section{Grasses}
[21] Sedge species*
[7] Slender wheatgrass
[8] Tufted hairgrass
[28] Kentucky bluegrass
[1] Fringed brome

\section{SITE CHARACTERISTICS}

Moisture regime: mesic, subhygric,hygric Nutrient regime: very rich Topographic position: lowerslope, level Slope: $0 \%, 5-40 \%$

Aspect: south westerly, south easterly, south and east

\section{SOIL CHARACTERISTICS}

Organic thickness: $(0-5)$

Humus form: moder

Surface texture: SiL, L

Effective texture: SiL, LS, L

Depth to Mottles/Gley: none

Drainage: moderately well, poor

Parent material: GL, GF, F

Soil subgroup: O.DYB, O.HR

\section{RANGE PLANT COMMUNITY TYPES}

UFC1. Slender wheatgrass-Sedge/Low forbs $n=9$ UFC3. Kentucky bluegrass/Dandelion $n=23$. UFC4. Kentucky bluegrass-Sedge/Dandelion $n=25$ UFC5. Tufted hairgrass-Kentucky bluegrass $n=4$ UFC6. Sedge/Tufted hairgrass $n=13$ 


\section{j2 Horsetail $\mathbf{P b}(\mathrm{n}=1)$}

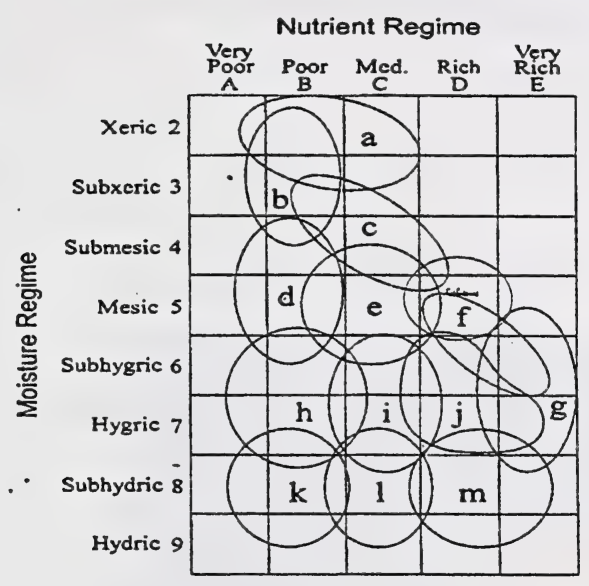

\section{SITE CHARACTERISTICS}

Moisture regime: hygric, subhygric, subhydric

Nutrient regime: rich, very rich, medium, poor Topographic position: toe, midslope, lowerslope, level

Slope: level, (2-\%)

Aspect: level, northerly, westerly

\section{SOIL CHARACTERISTICS}

Organic thickness: $(6-15)(0-5)$

Humus form: raw moder, mor, peatymor

Surface texture: SiL, L, SL

Effective texture: SiL, LS, L

Depth to Mottles/Gley: none, (26-50)(0-25)

Drainage: imperfect, poor, moderately well

Parent material: F, C

Soil subgroup: R.G, O.R, O.EB, GL.EB

\section{RANGE PLANT COMMUNITY TYPES}

UFD6. $\mathrm{Pb} /$ Willow/Horsetail $(\mathrm{n}=1)$

\section{CHARACTERISTIC SPECIES}

\section{Trees}

[35]

[5]

[3]

Shrub

Balsam poplar

Aspen

White spruce

[30] Willow

[3] Rose

Forb
[12] Horsetail
[9] Scouring rush
[3] Tall lungwort
[1] Scouring rush
[4] Clover
[7] Strawberry

\section{Grasses}

[1] Hairy wildrye

[1] Marsh reedgrass

[1] Kentucky bluegrass 


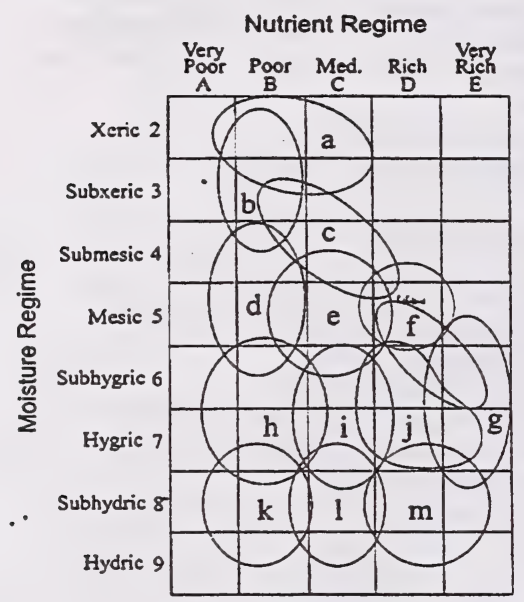

Moisture regime: hygric, subhygric

Nutrient regime: rich

Topographic position: level, midslope, lower slope, toe

Slope: (1)\%

Aspect: north

\section{SOIL CHARACTERISTICS}

Organic thickness: (6-15), (0-5)

Humus form: mor, raw moder, peatymor

Surface texture: SiL, SL, L

Effective texture: SiL, LS, L

Depth to Mottles/Gley: none

Drainage: imperfectly, moderately well, poor, very poor

Parent material: F, C

Soil subgroup: R.G, O.R, O.EB, GL.EB

\section{RANGE PLANT COMMUNITY TYPES}

\section{CHARACTERISTIC SPECIES}

Forb

UFF3. Sw/Horsetail/Kentucky bluegrass( $n=1)$
[18] Dandelion
[3] Strawberry
[22] Yarrow
[8] Graceful cinquefoil
[5] Veiny meadow rue

\section{Grasses}

[4] Creeping red fescue

[4] Slender wheatgrass

[46] Kentucky bluegrass 


\section{UPPER FOOTHILLS SUBREGION}

\section{NATIVE GRASSLANDS AND SHRUBLANDS}

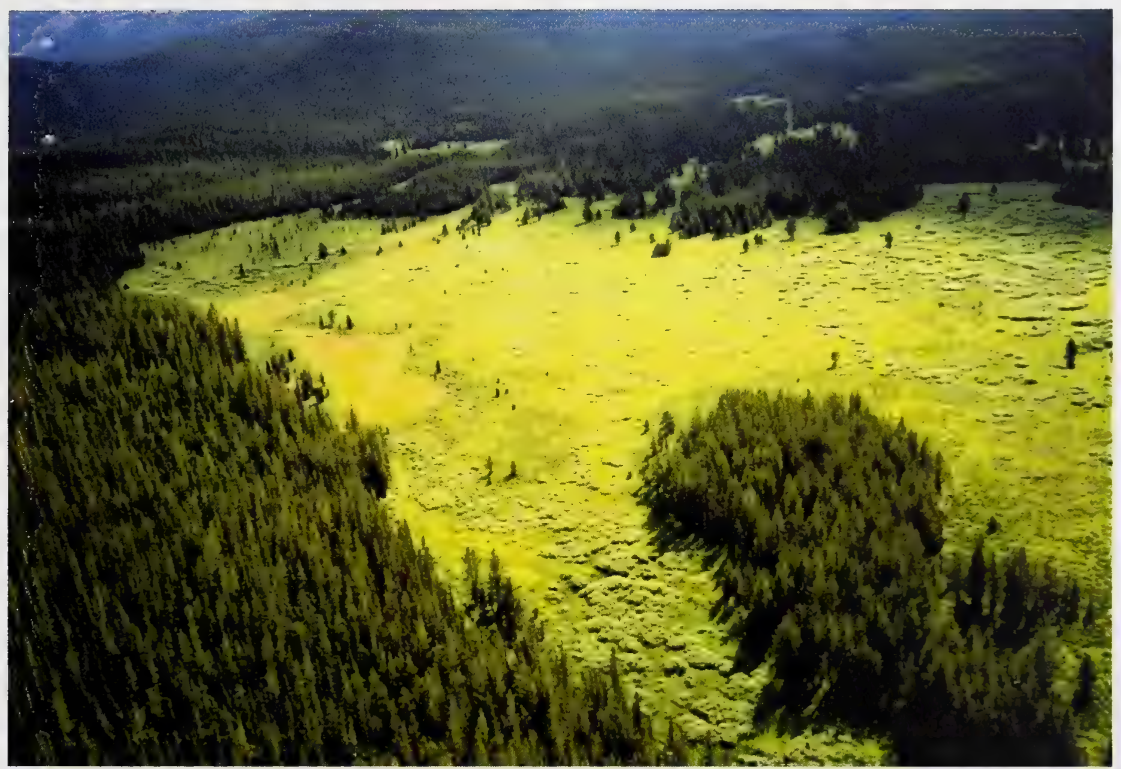

Figure 1. Overview of native shrub and grassland complex in the Upper Foothills subregion 



\section{Native grass and shrublands}

The native grass and shrubland community types (Table 2 ) are found in the valley bottoms, adjacent to streams and rivers, throughout theUpperFoothills subregion. Deep snow accumulations and cold air drainage prevent trees from growing in these valley bottoms (Daubenmire, 1978). Historically, these grass and shrublands burned frequently, further preventing tree encroachment.

The sequence of these community types along a moisture gradient from wet (UFA1 sedgemeadows) to dry (UFA9 junegrass-sedge/ sage slopes) is outlined in Figure 3. The change in species composition from the wet sedge meadows to rough fescue and California oatgrass meadows may occur over a 3 foot elevational gradient.

The maintenance of these grassland community types is extremely fire dependent. The lack of fire allows bog birch and willow to expand, shading the modal grassland community types. Prolonged shading causes the understory composition to shift from a tufted hairgrass-rough fescue dominated understory to one dominated by slender wheatgrass and sedge (Figure 3). Under heavy shrub cover (pussy willow shrubland and willow-bog birch community types), there is little forb or grass understory. Increased shrub cover also causes a decline in forage productivity and reduces the accessibility for livestock.

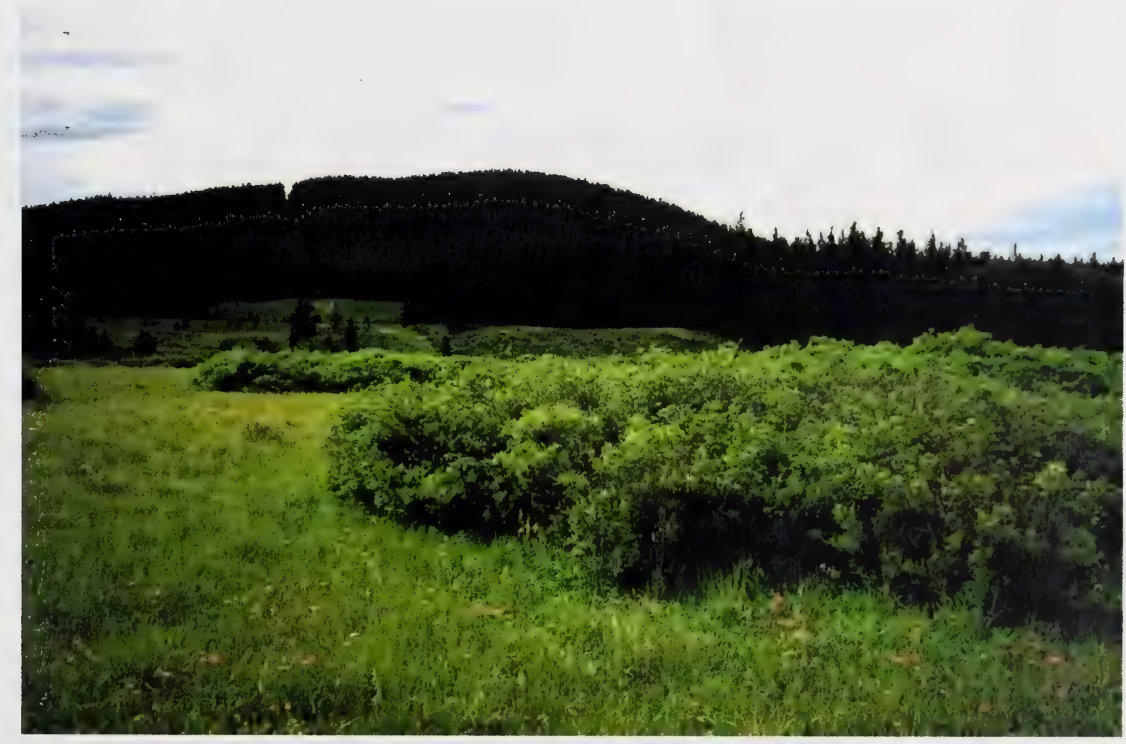

Figure 2. Typical native shrub and grassland transition zone in Upper Foothills subregion 



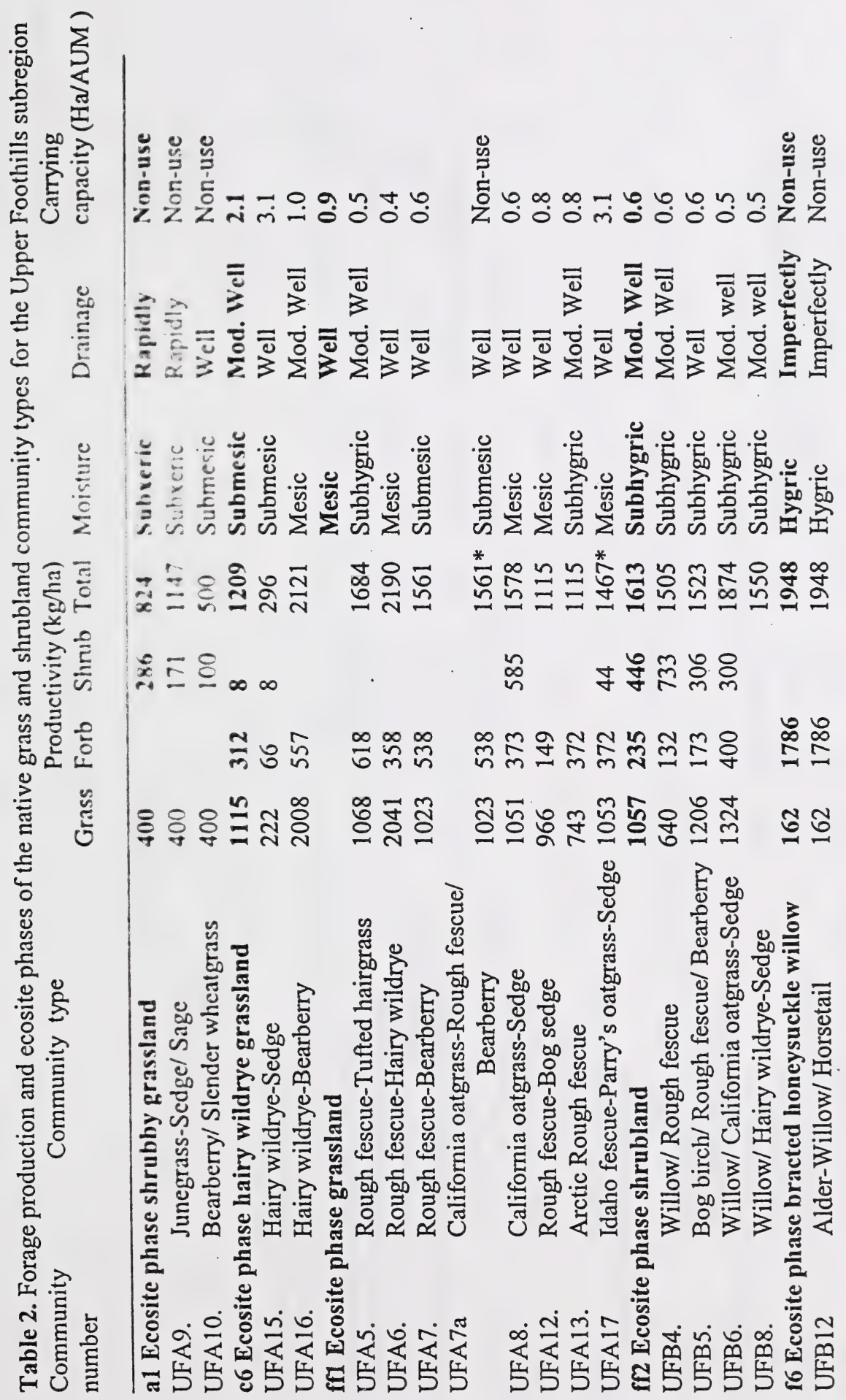




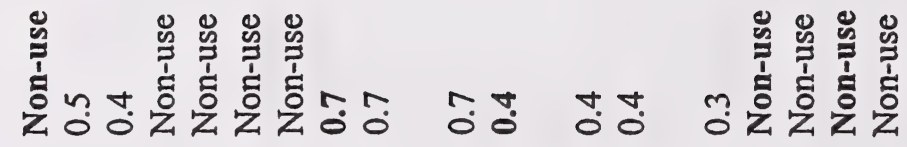

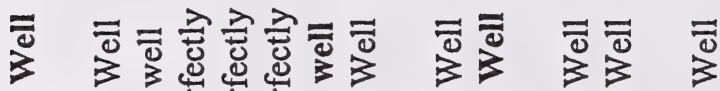

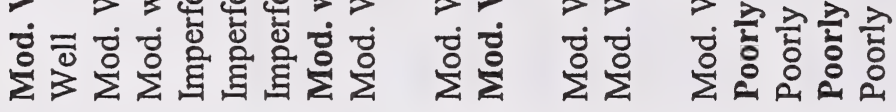

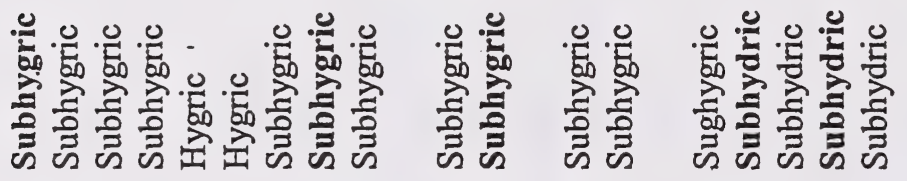

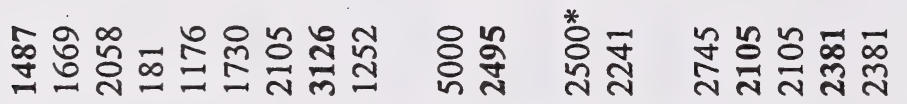

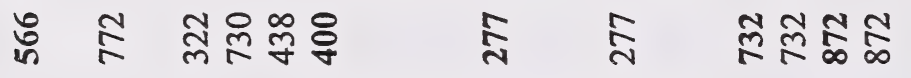

m

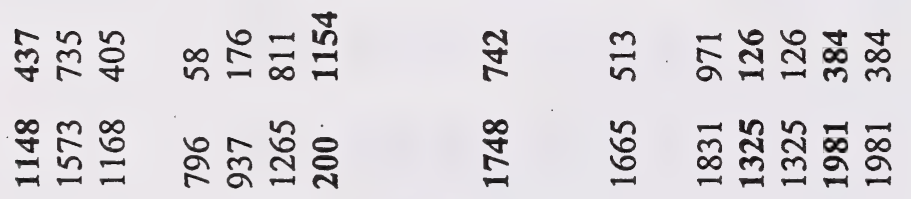

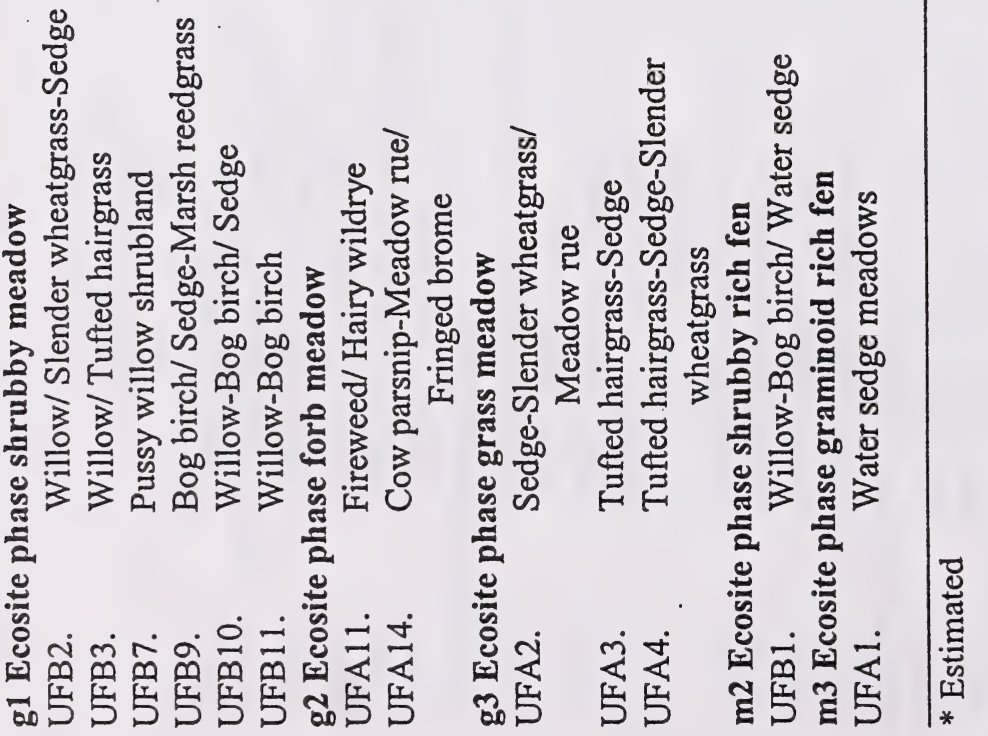



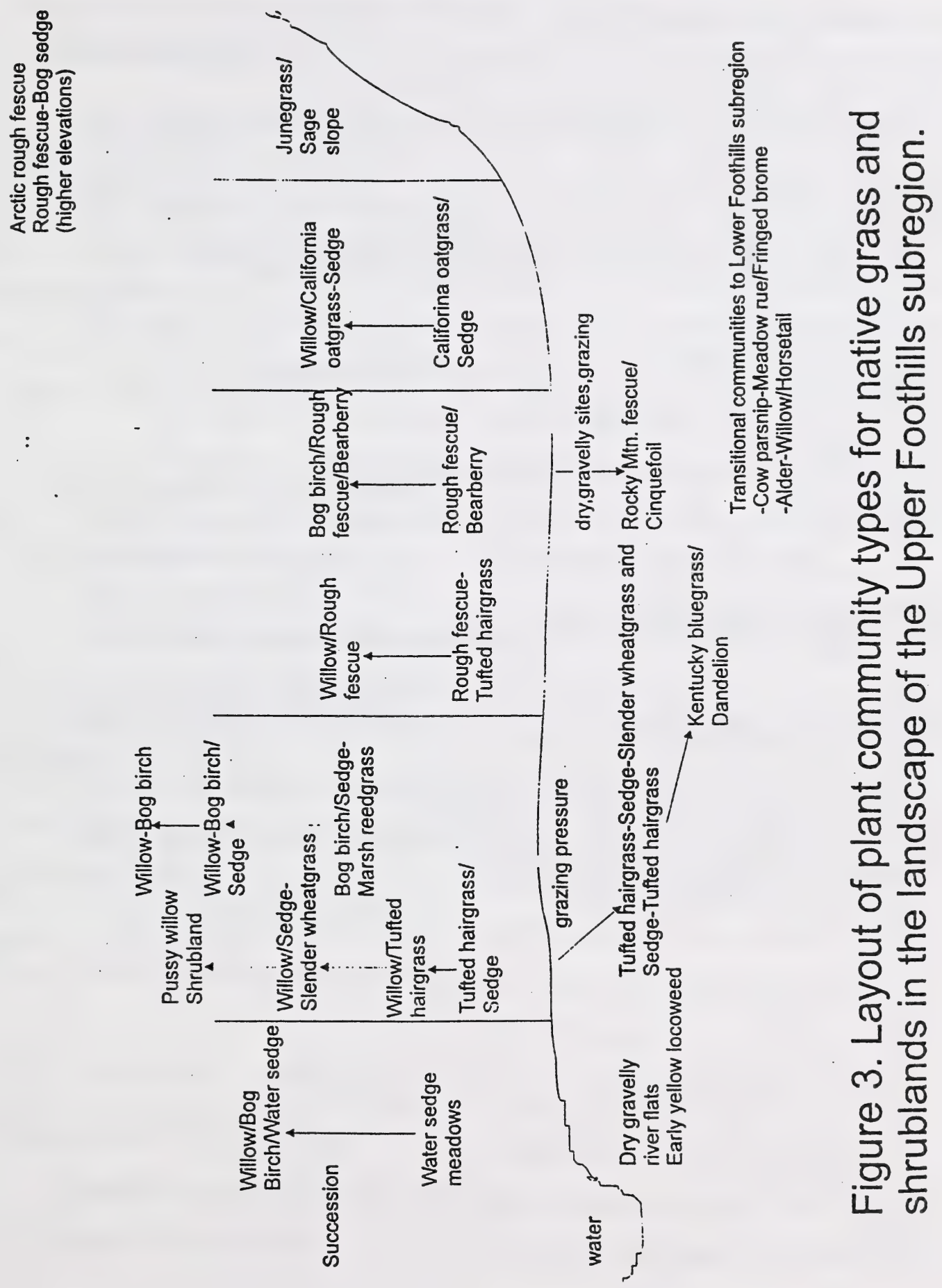


\section{Kev to Grassland Community Trpes (Grasslands dominated by native species)}

1. Hydric, periodic flooding, depressional, dominated by sedges $\ldots \ldots \ldots \ldots \ldots$ UFA1 Water sedge meadows Drier, ungrazed or lightly grazed dominated by forbs or grass (rough fescue, tufted hairgrass) $\ldots \ldots \ldots \ldots . .2$

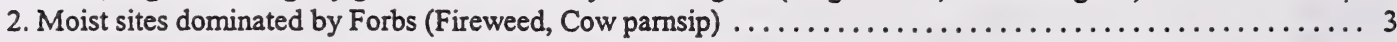

Grass dominated(upland sedges, rough fescue, slender wheatgrass, hairy wildrye, tufted hairgrass) ......4

3. Moist, lowland sites dominated by fireweed ........... UFA11 Fireweed/ Hairy wildrye (Forb meadow)

Fine textured, silty soils, dominated by cow parsnip ........ UFA14 Cow parsnip-Meadow rue/ Fringed brome

4. Moist sites dominated by Tufted hairgrass with no rough fescue present at site, includes moderately grazed sites

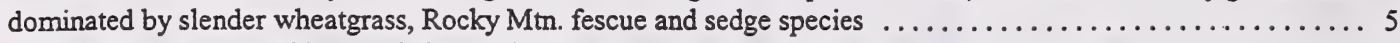

Drier sites dominated by rough fescue, hairy wildrye, california oatgrass, slender wheatgrass $\ldots \ldots \ldots \ldots 6$

5. Early successional sites with little slender wheatgrass, dominated by Tufted hairgrass

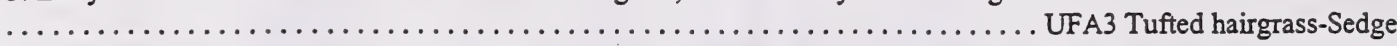

Later successional or grazed tufted hairgrass or rough fescue meadows with abundant forbs, sedge, and

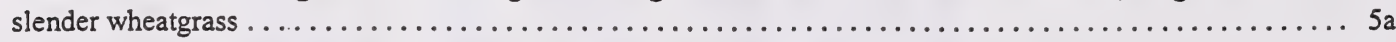

5a Lightly grazed sites dominated by tufted hairgrass, Sedge and Slender wheatgrass....

..UFA4 Tufted hairgrass-Sedge-Slender wheatgrass

Lightly to moderately grazed sites dominated by sedge, slender wheatgrass, Rocky Mtn. fescue or grareful cinquefoil.

5 b Moderately to heavily grazed site dominated by Rocky Mm. fescue or graceful cinquefoil.

.UFC2 Rocky Mtn. fescue/Graceful cinquefoil

Lightly to moderately grazed site dominated by slender wheatgrass, sedge, low forb species or rough fescue

5c Dry well drained sites with some rough fescue present..................UFC11Sedge-Slender wheatgrass-Rough fescue

Moister sites, tufted hairgrass present..............................................UFC1 Slender wheatgrass-Sedge/Low forbs

6. Rough fescue dominated, higher elevation and moist sites $\ldots \ldots \ldots \ldots \ldots \ldots \ldots \ldots \ldots \ldots \ldots \ldots \ldots$

Hairy wildrye, California oatgrass, slender wheatgrass, Idaho fescue, Parry oatgrass or Purple oatgrass

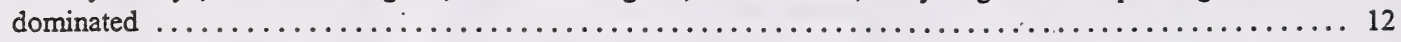

7. Moist Alpine sites with globeflower, fleabane, monkshood, mountain heliotrope or drier sites with bog sedge .. 8

Drier sites at lower elevations tufted hairgrass, hairy wildrye, bearberry or slender wheatgrass codominant .... 9

8. Dry well drained sites with bog sedge codominant .............. UFA12 Rough fescue-Bog sedge

Moist Alpine sites with alpine forb species $\ldots \ldots \ldots \ldots \ldots \ldots \ldots \ldots \ldots$ UFA13 Alpine rough fescue

9.Moist sites codominated with Tufted hairgrass ............... UFA5 Rough fescue-Tufted hairgrass

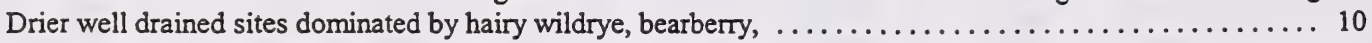

10. Lower, south facing slopes, well-developed soils hairy wildrye codominant . . UFA6 Rough fescue-Hairy wildrye

Well drained sites with Bearberry present in understory $\ldots \ldots \ldots \ldots \ldots \ldots \ldots \ldots \ldots \ldots \ldots \ldots \ldots \ldots$

11. Shallow, well drained, gravelly soils, low nutrient .............. UFA7 Rough fescue/ Bearberry Ghost area California oatgrass dominated .......... UFA7a California oatgrass-Rough fescue/ Bearberry

12. California oatgrass dominated, well-drained soil, cold air drainage level areas in valley bottoms

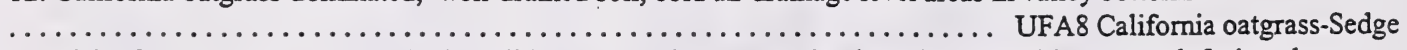
Idaho fescue, Parry oatgrass, Hairy wildrye or Purple oatgrass dominated communities on south facing slopes or

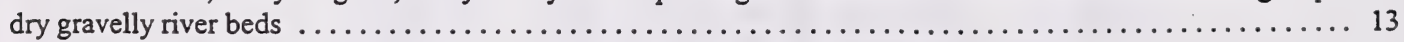

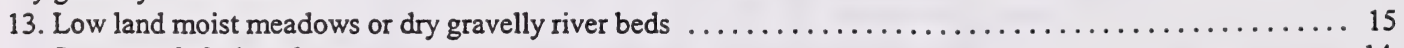
Steep south facing slopes...............................................................................................................................14

14. Steep, south facing slopes, shallow soils, drought tolerant species Junegrass, sage UFA9 Junegrass-Sedge/ Sage Hairy wildrye or Idaho fescue, Purple oatgrass, Parry oatgrass dominated sites $\ldots \ldots \ldots \ldots \ldots \ldots \ldots 16$

15. River bed, dry, gravelly, well drained sites ............... UFA10 Bearberry/ Slender wheatgrass Moist meadows dominated by drier sedge species ......... UFA2 Sedge-Slender wheatgrass/ Meadow rue

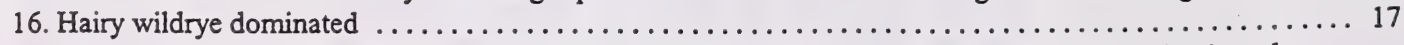
Moister, south facing slopes in the Ghost area, Idaho fescue, Parry oatgrass, Purple oatgrass dominated

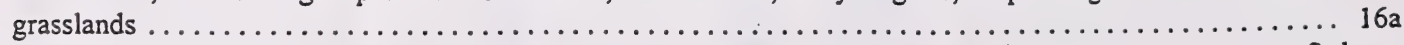

16a Idaho fescue, Parry oatgrass dominated sites in the Ghost area.................UFA17 Idaho fescue-P.oatgrass-Sedge 
Grazed sites dominated by Purple oatgrass and rough fescue. UFC9 Purple oatgrass-Rough fescue

17. South facing slopes, at higher elevation UFA15 Hairy wildrye-Sedge

Grazed site, dominated by hairy wildrye, rough fescue present .. UFA16 Hairy wildrye-Rough fescue/Bearberry

Key to Grazing Modified Grassland and Shrubland Community Types (dominated by non-native species)

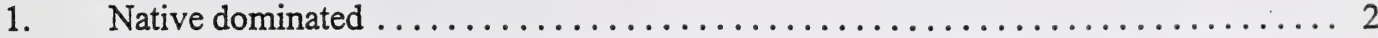

Non-native dominated, creeping red fescue, Kentucky bluegrass, clover, dandelion . . 7

2. Grass dominated (slender wheatgrass, rocky mtn. fescue, sedge, purple oatgrass, tufted

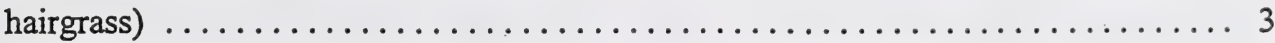

Shrub dominated with K. bluegrass understory .... UFC10 Willow/ Kentucky bluegrass

3. Moist grassy meadows with rough fescue, tufted hairgrass still present on site ...... 4 Drier, well drained sites dominated by Rocky mtn. fescue

$\ldots \ldots \ldots \ldots \ldots \ldots \ldots \ldots \ldots \ldots$ UFC2 Rocky mtn. Fescue/ Graceful cinquefoil

4 Slender wheatgrass, Sedge and purple oatgrass dominated community $4 a$

Grazed tufted hairgrass communities(moister sites)..........................................................5

4a Slender wheatgrass and Sedge dominated.......UFC1 Slender wheatgrass-Sedge/Low forbs Purple oatgrass dominates. UFC9 Purple oatgrass-Rough fescue

3. Kentucky bluegrass present .......... UFC5 Tufted hairgrass-Kentucky bluegrass Kentucky bluegrass absent, recovering site ......... UFC6 Sedge-Tufted hairgrass

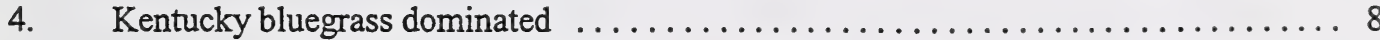

Seeded sites with Creeping red fescue ......... UFC7 Creeping red fescue/ Clover

5. Heavily grazed sites with dandelion as co-dominant

UFC3 Kentucky bluegrass/ Dandelion

Heavily grazed cow parsnip meadow, lower elevation sites, cow parsnip present in small amounts

UFC8 Kentucky bluegrass-Timothy/ Meadow rue 


\section{Key to Shrubland Community Types}

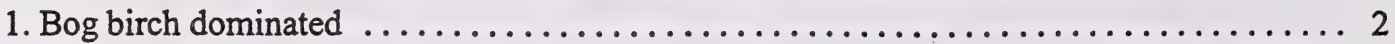

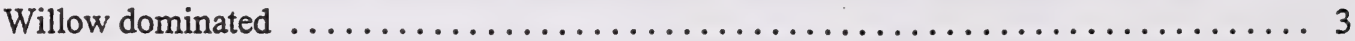

2. Dry, nutrient poor soils, with rough fescue ...... UFB5 Bog birch/ Rough fescue/ Bearberry Wet, poor drainage, no willow cover .......... UFB9 Bog birch/ Sedge-Marsh reedgrass

3. Poorly drained sites with water sedge in understory, or shrublands with little understory . . 4 Drier well drained sites with slender wheatgrass, hairy wildrye, rough fescue, tufted hairgrass

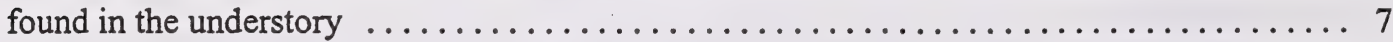

4. Poor drainage, very wet sites ............. UFB1 Willow-Bog birch/Water sedge Shrub dominated sites with little understory willow and bog birch dominated ......... 5

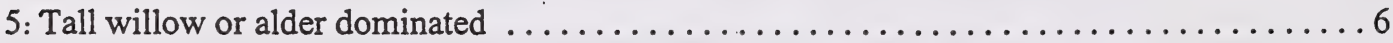
Short willow, imperfectly drained sites little understory ........ UFB11 Willow-Bog birch

6. Occurring along water bodies $\ldots \ldots \ldots \ldots \ldots \ldots \ldots$ UFB7 Pussy willow shrubland Moist, nutrient rich seepage sites with alder and willow .... . UFB12 Alder-Willow/ Horsetail

7. Well drained sites with Hairy wildrye dominating the understory, typical of well drained valley bottomland sites $\quad \ldots \ldots \ldots \ldots \ldots \ldots \ldots \ldots$ UFB8 Willow/ Hairy wildrye-Sedge

Recently invaded grasslands with rough fescue, tufted hairgrass or california oatgrass, slender wheatgrass or graceful sedge $\ldots \ldots \ldots \ldots \ldots \ldots \ldots \ldots \ldots \ldots \ldots \ldots$

8. California oatgrass dominated ............ UFB6 Willow/ California oatgrass-Sedge Rough fescue or tufted hairgrass present in understory .................. 9

9. Rough fescue dominated..$\ldots \ldots \ldots \ldots \ldots \ldots \ldots$ UFB4 Willow/ Rough fescue Tufted hairgrass, graceful sedge or slender wheatgrass dominated ............ 10

10. Tufted hairgrass dominated $\ldots \ldots \ldots \ldots \ldots \ldots \ldots$ UFB 3 Willow/ Tufted hairgrass Sedge or Slender wheatgrass dominated understory................................................................11

11. Sedge dominated .................... UFB10 Willow-Bog birch/ Sedge Slender wheatgrass dominated $\ldots \ldots \ldots \ldots \ldots$ UFB2 Willow/ Slender wheatgrass-Sedge 


\section{UFA1. Water-Beaked sedge meadows (Carex aquatilis-Carex rostrata)}

$\mathrm{n}=14$ Wet conditions and periodic flooding result in the formation of water sedge meadows. Bog birch and willow will invade into the drier edges of these meadows to form the willow-bog birch/water sedge community type (UFB1).

These community types are quite productive, producing nearly $2000 \mathrm{~kg} / \mathrm{ha}$ of forage, but the high water table in the spring and summer when these meadows are most palatable limits livestock use. A study in the Yukon found that crude protein on these meadows declined from a high of $10 \%$ in May to less than $5 \%$ in September (Bailey et al., 1992). As a result, these meadows would be rated as secondary or non-use range.

\section{PLANT COMPOSITION CANOPY COVER(\%) Mean Range Const.}

SHRUBS

WILLOW

(Salix spp)

$2 \quad 0-10 \quad 79$

BOG BIRCH

$\begin{array}{llll}\text { (Betula glandulosa) } & 1 & 0-1 & 29\end{array}$

FORBS

ARROW LEAVED COLTSFOOT

(Petasites sagittatus) 2

GRASSES

WATER SEDGE

(Carex aquatilis)

BEAKED SEDGE

(Carex rostrata)

SEDGE

(Carex spp)

TUFTED HAIRGRASS

(Deschampsia cespitosa)
$14 \quad 0-63 \quad 29$

$2 \quad 0-30 \quad 7$

$64 \quad 0-96 \quad 50$

$0-40 \quad 86$

\section{ENVIRONMENTAL VARIABLES}

MOISTURE REGIME:

SUBHYDRIC

NUTRIENT REGIME MESOTROPHIC

ELEVATION:

$1091-1760 \mathrm{M}(1484 \mathrm{M})$

SOIL DRAINAGE:

POORLY

ASPECT:

VARIABLE

SLOPE:

$0-5 \%$

FORAGE PRODUCTION(KG/HA)

GRASS: 1981 (810-4438)

FORBS: 384 (46-776)

SHRUBS: 872 (8-1736)

TOTAL: 2381 (966-4684)

\section{SUGGESTED GRAZING CAPACITY NON-USE}




\section{UFA2. Sedge-Slender wheatgrass/ Meadow rue \\ (Carex spp.-Agropyron trachycaulum/ Thalictrum venulosum)}

$\mathrm{n}=3$ These sites are dominated by three sedge species that are adapted to moist conditions: $C$. praticola, $C$. praegracilus and $C$. prairea. The presence of small amounts of tufted hairgrass and rough fescue indicates that these sites may represent a phase of the rough fescue-tufted hairgrass plant community (UFA5). Past heavy grazing pressure may have shifted the plant community to one dominated by sedge species or these sites could be too wet for tufted hasgrass and rough fescue growth.

The furage productivity on this community type is good. In comparison with the water sedge meadows (UFAl), these sites remain drier throughout the growing season and this allows easy access for livestock. This communty would be rated as primary range.

\section{PLANT COMPOSITION CANOPY COVER(\%)}

MEan Range CONST.

SHRLBS

SHRUBBY CPror EPOLL

(Potenulle frusicosa)

BOG B!RCH

(Berula glonduiosa)

VEINY MEADO H RLE

(Thalictrum vernulosum) $28 \quad 20-36 \quad 100$

OLDMAN'S WHSSERS

(Geum triflorum) 9

(Penstemon procerus) 5

YARROW

(Achillea millefolium)

SILVERY CRNQUEFOIL

(Potentilla arguta)

LNDLEY'S ASTER

(Aster ciliolatus)

GRASSES

MEADOW SEDCE

(Carex praticola)

GRACEFUL SEDGE

(Carex praegracilis) $\quad 11 \quad 0-32 \quad 33$

PRAIRIE SEDGE

(Carex prairea)

SEDGE

(Carex spp)

$9 \quad 0-28 \quad 33$

25

SLENDER WHEATGRASS

(Agropyron trachycaulum) 8
$0-2 \quad 67$

$0-2 \quad 33$

$0-14 \quad 67$

$0-8 \quad 67$

$5-14 \quad 100$

$0-8 \quad 67$

$0-5 \quad 33$

$0-26 \quad 67$

$0-75 \quad 33$

$0-12 \quad 67$

\section{ENVIRONMENTAL VARIABLES}

MOISTURE REGIME:

SUBHYGRIC

NUTRIENT REGIME:

PERMESOTROPHIC

ELEVATION:

$1460 \mathrm{M}$

SOIL DRAINAGE:

MODERATELY WELL

ASPECT:

EAST

SLOPE:

$0-5 \%$

FORAGE PRODUCTION(KG/HA)

TOTAL: 2500

\section{SUGGESTED GRAZING CAPACITY} 0.4 HAAUM OR 0.8 ACRES/AUM 


\section{UFA3. Tufted hairgrass-Sedge \\ (Deschampsia cespitosa-Carex praegracilis)}

$\mathbf{n}=34$ This community is located on moist sites that are better drained and slightly drier than the pure sedge meadows (UFA1). Willoughby (1992) and Willoughby (1999), found that tufted hairgrass is a common plant species on these lowland sites throughout the Upper Foothills and Lower Subalpine subregions. At lower elevations, this species appears to be replaced by marsh reedgrass. When this community type is protected from grazing for 25-30 years, willow and bog birch expand (willow/ tufted hairgrass (UFB3)) and tufted hairgrass and sedge decline (Willoughby, 1992). The decline in graminoid cover also results in a decline in available forage production from 2200 to $1800 \mathrm{~kg} / \mathrm{ha}$. Continuous heavy grazing pressure causes hairgrass to decline and the site will be invaded by Kentucky bluegrass and dandelion.

Bork (1994), found this community type. to be the most productive type described in Willmore wilderness park. Forage production averages over $2000 \mathrm{~kg} / \mathrm{ha}$ and can vary from $800-3300 \mathrm{~kg} / \mathrm{ha}$. This community type would be rated as primary range.

PLANT COMPOSITION Canopy CoVER(\%) MEAN RANGE CONST.

\section{SHRUBS}

BARCLAY'S WILLOW

(Salix barclayi)

WILLOW

(Salix spp)

BOG BIRCH

(Betula glandulosa) $\quad 1 \quad 0-10 \quad 23$

FORBS

YARROW

(Achillea millefolium)

(Fragaria virginiana)

VEINY MEADOW RUE

(Thalictrum venulosum)
LINDLEY'S ASTER

(Aster ciliolatus)

GRACEFUL CINQUEFOIL

(Potentilla gracilis)

DANDELION

(Taraxacum officinale)

GRASSES

TUFTED HAIRGRASS

(Deschampsia cespitosa) $31 \quad 2-70 \quad 100$

GRACEFUL SEDGE

$\begin{array}{llll}\text { (Carex praegracilis) } & 12 & 0-43 \quad 69\end{array}$

WATER SEDGE

(Carex aquatilis)

SEDGE

(Carex spp)

SLENDER WHEATGRASS $\begin{array}{lll}\text { (Agropyron trachycaulum) } 9 & 0-27 & 71\end{array}$

\section{ENVIRONMENTAL VARIABLES}

MOISTURE REGIME:

SUBHYGRIC

NUTRIENT REGIME:

PERMESOTROPHIC

ELEVATION:

1276-1800 M (1461 M)

SOIL DRANAGE:

MODERATELY WELL

ASPECT:

VARIABLE

SLOPE:

$0-40 \%$

\section{FORAGE PRODUCTION(KG/HA)}

GRASS: 1665 (422-3654)

FORBS: $513(6-1572)$

SHRUBS: 277 (124-414)

TOTAL: $2455(824-3706)$

\section{SUGGESTED GRAZING CAPACITY}

$0.4 \mathrm{H}$ VAUM OR 0.9 ACRES/AUM 


\section{UFA4. Tufted hairgrass-Sedge-Slender wheatgrass \\ (Deschampsia cespitosa-Carex spp.-Agropyron trachycaulum)}

$\mathrm{n}=\mathbf{8}$ This community type may be transitional between the willow dominated community types and the tufted hairgrass dominated grasslands. Two of the sites described in this community are represented by the inside, ungrazed transect at two rangeland reference area sites. Protection from grazing for 25-35 years appears to allow willow to expand and there is a shift away from a tufted hairgrass dominated community type to a type that is dominated by slender wheatgrass, sedge and tall forb species. Continued protection from grazing and fire will likely lead to a community dominated by willow and bog birch with little understory of forbs and grass.

PLANT COMPOSITION CANOPY COVER(\%) MEAN RANGe CONST.

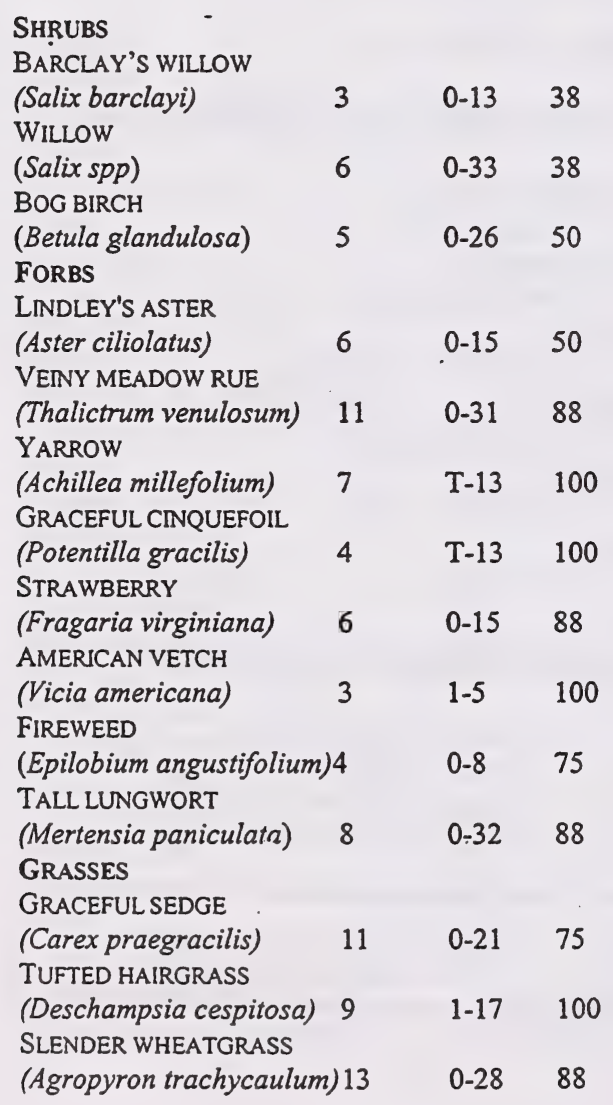

\section{ENVIRONMENTAL VARIABLES}

MOISTURE REGIME:

SUBHYGRIC

NUTRIENT REGIME:

PERMESOTROPHIC

ELEVATION:

1303-1805 M (1423 M)

SOIL DRANAGE:

MODERATELY WELL

ASPECT:

SLOPE:

VARLABLE

$0-5 \%$

\section{FORAGE PRODUCTION(KG/HA)}

GRASS: $1831(864-2416)$

FORBS: 971 (477-1702)

TOTAL: $2745(1478-4118)$

SUGGESTED GRAZING CAPACITY 0.3 HAAUM OR 0.7 ACRES/AUM 


\section{UFA5. Rough fescue-Tufted hairgrass \\ (Festuca scabrella-Deschampsia cespitosa)}

$\mathrm{n}=5$ This community type is located up slope from the tufted hairgrass-sedge community type (UFA3) on drier, better drained soils. The drier soil conditions limit the amount of forage being produced. There was $300 \mathrm{~kg} / \mathrm{ha}$ less forage produced in the rough fescue-tufted hairgrass community type compared to the tufted hairgrass-sedge community type (UFA.3).

In the absence of fire and grazing, this community type will become dominated by willow and bog birch (willow/ rough fescue community type (UFB4)). Heavy grazing pressure also decreases the cover of rough fescue and tufted hairgrass and allows Kentucky bluegrass and dandelion to increase (Willoughby, 1992). The dominant plant species on this community are highly palatable and the sites are easily accessible to livestock. Consequently, this community would be rated as primary range.

\section{Plant CoMtPosition Canopy Cover(\%) Mean Range Const.}

\section{FORBS}

SLENDER BLUE BEARDTONGUE

(Penstemon procerus) 4

YARROW

(Achillea millefolium) $4 \quad 1-11 \quad 100$

GRACEFUL CINQUEFOIL

$\begin{array}{llll}\text { (Potentilla gracilis) } & 3 & 0-6 & 80\end{array}$

CHICKWEED

(Cerastium arvense) $\quad 1 \quad 0-4 \quad 80$

MONKSHOOD

(Aconitum delphinfolium) 1

OLD MAN'S WHISKERS

(Geum triflorum)

GRASSES

ROUGH FESCUE

(Festuca scabrella)

TUFTED HAIRGRASS

(Deschampsia cespitosa) $17 \quad 3-29 \quad 100$

SLENDER WHEATGRASS

(Agropyron trachycaulum) $4 \quad 1-6 \quad 100$

GRACEFUL SEDGE

(Carex praegracilis)

13

CALIFORNIA OATGRASS

(Danthonia californica)

\section{ENVIRONMENTAL VARIABLES}

MOISTURE REGIME:

SUBHYGRC

NUTRIENT REGIME:

PERMESOTROPHIC

ELEVATION:

1370-1737 M (1532 M)

SOIL DRAINAGE:

MODERATELY WELL

ASPECT:

SOUTHEAST

SLOPE:
$0-3 \%$

FORAGE PRODUCTION (KG/HA)

GRASS: $1068(605-1797)$

FORBS: $618(166-1252)$

TOTAL: $1684(913-2272)$

\section{SUGGESTED GRAZING CAPACITY} 0.5 HAAUM OR 1.2 ACRES/AUM 


\section{UFA6. Rough fescue-Hairy wildrye \\ (Festuca scabrella-Elymus innovatus)}

$\mathrm{n}=18$ These grasslands are located on lower, south facing slopes. They represent the transition zone from the dry junegrass-sedge/ sage (UFA9) dominated south facing slopes to the moist rough fescue and tufted hairgrass dominated community types (UFA5). Grazing pressure causes a shift away from a rough fescue, hairy wildrye dominated community (UFA6) to a sedge, Kentucky bluegrass dominated community (UFC4) (Willoughby, 1992). These grasslands are fairly moist and have well developed soils which makes them very productive. This community type would be rated as primary range.

This community type is very similar to the rough fescue dominated communities described in the $\mathrm{Ya} \mathrm{Ha}$ Tinda, west of Sundre (Willoughby et al. 2001)

Plant COMPOSITION CANOPY COVER(\%) Mean Range Const.

SḦRUBS

BEBB'S WILLOW

(Salix bebbiana)

SHRUBBY CINQUEFOIL

$\begin{array}{llll}\text { (Potentilla fruticosa) } & 3 . & 0-13 & 78\end{array}$

FORBS

FIREWEED

(Epilobium angustifolium) $3 \quad 0-30 \quad 27$

OLD MAN'S WHISKERS

(Geum triflorum) $\quad 5 \quad 0-19 \quad 56$

STAR FLOWERED SOLOMON'S SEAL

(Smilacina stellata)

(Fragaria virginiana)

VEINY MEADOW RUE

(Thalictrum venulosum) 4

GRASSES

ROUGH FESCUE

(Festuca scabrella)

HAIRY WILDRYE

(Elymus innovatus)

$2 \quad 0-27 \quad 33$

$4 \quad 0-9 \quad 67$

SLENDER WHEATGRASS

(Agropyron trachycaulum)

KENTUCKY BLUEGRASS

(Poa pratensis)

GRACEFUL SEDGE

(Carex praegracilis)

SEDGE

(Carex spp)

PRAIRIE SEDGE

(Carex prairea)

JUNEGRASS

(Koeleria macrantha) $\begin{array}{lll}35 & 8-85 \quad 100\end{array}$

$\begin{array}{lll}8 & 0-28 \quad 67\end{array}$

$0-18 \quad 61$

$0-5 \quad 39$

$0-42 \quad 22$

$0-24 \quad 44$

$0-18 \quad 11$

$0-19 \quad 67$

\section{ENVIRONMENTAL VARIABLES}

MOISTURE REGIME:

MESIC

NUTRIENT REGIME:

MESOTROPHIC

ELEVATION:

1320-1798 M (1589 M)

SOIL DRAINAGE:

WELL

ASPECT:

SLOPE:

SOUTH

$5-48 \%$

FORAGE PRODUCTION (KG/HA)

GRASS: $2041(472-4832)$

FORBS: $358(12-696)$

TOTAL: $2190(484-5162)$

SUGGESTED GRAZING CAPACITY 0.4 HNAUM OR 0.9 ACRES/AUM 


\section{UFA7. Rough fescue/ Bearberry \\ (Festuca scabrella/ Arctostaphylos uva-ursi)}

$\mathrm{n}=4$ This community type was described in the Upper Clearwater Forest Land Use Zone and is similar to the bog birch' rough fescue/ bearberry community type but lacks the cover of bog birch. Willoughby (2001) felt that bog birch indicated sites with deeper snow accumulations. This community occupies sites that have shallow, welldrained, and gravelly soils and there maybe little snow cover which does not favour the growth of bog birch. This communsty is moderately productive but because of the poor soil conditions, precautions must be taken to prevent overutilization

PLANT COUPOSITION CANOPY COVER(\%)

\section{Mean Range Const.}

\begin{tabular}{|c|c|c|c|}
\hline \\
\hline \multicolumn{4}{|l|}{ BEARBERRY } \\
\hline (Árclostapholus who ursi) & 21 & $12-31$ & 100 \\
\hline SHRUBBY CDE EROIL & & & \\
\hline (Potentilia prusiona) & 2 & $0-5$ & 75 \\
\hline FORBS & & & \\
\hline OLDMAN'S WHYKERS & & & \\
\hline (Geum triflorwm) & 10 & $0-26$ & 75 \\
\hline YARROW & & & \\
\hline (Achillea milirium) & 11 & $0-38$ & 75 \\
\hline GRACEFUL CNOCFO:L & & & \\
\hline (Potenullagracitis) & 3 & $0-10$ & 50 \\
\hline Grasses & & & \\
\hline ROUGH FESCUE & & & \\
\hline (Festuca scabrella) & 49 & $38-56$ & 100 \\
\hline SLENDER KHEATGRASS & & & \\
\hline (Agropyron trachycaulum) & & $5-19$ & 100 \\
\hline GRACEFUL SEDCE & & & \\
\hline (Carex praegracilis) & 3 & $0-6$ & 75 \\
\hline FRINGED BROME & & & \\
\hline (Bromus ciliatus) & 4 & $0-7$ & 75 \\
\hline HAIRY WILDRYE & & & \\
\hline (Elymus innovaius) & 4 & $0-9$ & 75 \\
\hline
\end{tabular}

\section{ENVIRONMENTAL VARIABLES}

MOISTURE REGIME:

SUBXERIC

NUTRIENT REGIME:

MESOTROPHIC

ELEVATION:

1676-1829 M (1745 M)

SOIL DRAINAGE:

WELL

ASPECT:

SOUTH

SLOPE:

$0-5 \%$

Forage Production (KG/HA)

GRASS: $1023(580-1686)$

FORBS: $538(204-820)$

TOTAL: $1561(1156-1890)$

\section{SUGGESTED GRAZNG CAPACITY}

$0.6 \mathrm{HA} A U M$ OR 1.3 ACRES/AUM 


\section{UFA7a. California oatgrass-Rough fescue/ Bearberry \\ (Danthonia californica-Festuca scabrella/ Arctostaphylos uva-ursi)}

$\mathbf{n}=2$ This community type was described in the Ghost area west of Calgary. It appears to represent a transitional grassland between the Montane and Upper Foothills subregions. This community also appears to be transitional between the California oatgrass dominated grasslands (UFA8) and the previously described Rough fescue/Bearberry dominated community. This community is located on steep, south facing slopes and small hillcrests with welldrained subxeric soils. The dry site conditions limit the amount of forage available for domestic livestock and the steep slopes restrict livestock access. Consequently, this community type should be rated non-use.

PLANT COMPOSITION CANOPY COVER(\%) Mean Range Const.

\section{SHRUBS}

BEARBERRY -

(Arctostaphylos uva-ursi) $21 \quad 4-36 \quad 100$

SHRUBBY CINQUEFOIL

(Potentilla fruticosa) $\quad 8 \quad 1-15 \quad 100$

FORBS

OLD MAN'S WHISKERS

(Geum triflorum)

YARROW

(Achillea millefolium)

GRACEFUL CINQUEFOIL

(Potentilla gracilis)

GRASSES

CALIFORNIA OATGRASS

$\begin{array}{llll}\text { (Danthonia californica) } & 29 & 27-30 & 100\end{array}$

ROUGH FESCUE

$\begin{array}{llll}\text { (Festuca scabrella) } & 15 & 7-22 & 100\end{array}$

IDAHO FESCUE

(Festuca idahoensis) $\quad 8 \quad 5-11 \quad 100$

SEDGE

(Carex spp)

HAIRY WILDRYE

(Elymus innovatus)

$20 \quad 11-27 \quad 100$

T-3 100

T-1 100

$11 \quad 4-7 \quad 100$

$1 \quad 0-2 \quad 50$

MOISTURE REGIME: SUBXERIC

NUTRIENT REGIME: MESOTROPHIC

ELEVATION:

$1745 \mathrm{M}$

SOIL DRAINAGE:

WELL

ASPECT:

SOUTH

SLOPE:

$25 \%$

FORAGE PRODUCTION (KG/HA)

TOTAL 1561 *ESTMATE

\section{SUGGESTED GRAZING CAPACITY \\ 0.6 HAVAUM OR 1.3 AC/AUM}




\section{UFA8. California oatgrass-Sedge \\ (Danthonia californica-Carex praegracilis)}

$\mathrm{n}=9 \quad$ Dry, gravelly or stony soils support this moderately productive grassland that is dominated by California oatgrass. Small pockets of this community type occur throughout the Upper Foothills subregion. In the Yukon, these small meadows were found to form in depressions which appeared to act as pronounced frost pockets (Bailey et al., 1992). In the Subalpine subregion, these California oatgrass dominated grasslands are often associated with bog sedge (Willoughby 1999). The cold air drainage and poor nutrient quality of the soil limits the forage productivity of these sites.

\section{PLANT COMPOSITION CANOPY COVER(\%) Mean Range Const.}

SHRUBS

SHRUBBY CINQOEFOIL

(Potentilla fruticosa) $7 \quad 0-25 \quad 60$

DWARF BILBERRY

(Vaccinium caespitosum) $110-5 \quad 10$

FORBS

LINDLEY'S ASTER

(Aster ciliolatus)

$2 \quad 0-10 \quad 30$

WILD STRAWBERRY

(Fragaria virginiana)

OLD MAN'S WHISKERS

(Geum triflorum)

ALPINE MILK VETCH

(Astragalus alpinus)

VENY MEADOW RUE

(Thalictrum venulosum)

BLUE EYED GRASS

(Sisyrinchium montanum) 2

GRASSES

CALIFORNIA OATGRASS

(Danthonia californica)

GRACEFUL SEDGE

(Carex praegracilis)

SEDGE

(Carex spp)

$\begin{array}{lll}8 & 2-15 \quad 100\end{array}$

$14 \quad 0-46 \quad 90$

$3 \quad 0-17 \quad 30$

$\begin{array}{lll}7 & 0-25 \quad 90\end{array}$

$0-19 \quad 30$

SLENDER WHEATGRASS

(Agropyron trachycaulum) $8 \quad 0-36 \quad 80$

SHEEP FESCUE

(Festuca saximontana) $3 \quad 0-15 \quad 40$

COLUMBIA NEEDLEGRASS

(Stipa columbiana) $\begin{array}{lll}31 & 0-57 \quad 90\end{array}$

$10 \quad 0-37 \quad 50$

$18 \quad 0-50 \quad 50$

$0-21 \quad 30$
MOISTURE REGIME:

MESIC

NUTRIENT REGIME:

MESOTROPHIC

ELEVATION:

1400-1580 M (1484 M)

SOIL DRAINAGE:

WELL

ASPECT:

VARIABLE

SLOPE:

$0-45 \%$

\section{FORAGE PRODUCTION (KG/HA)}

GRASS: 1051 (400-1582)

FORBS: 373 (118-762)

SHRUBS: 585 (110-1402)

TOTAL: 1578 (1033-2014)

\section{SUGGESTED GRAZNG CAPACITY}

0.6 HAAUM OR 1.3 ACRES/AUM

\section{ENVIRONMENTAL VARIABLES}




\section{UFA9. Junegrass-Sedge/ Sage \\ (Koeleria macrantha-Carex spp./Artemisia frigida)}

$\mathrm{n}=4$. This community type occurs on steep, south facing slopes with shallow soils overlying sandstone bedrock. The majority of the vegetation is composed of the drought tolerant species: sage, bearberry and junegrass. The inaccessibility and fragile nature of the soils make this community type unsuitable for grazing.

This community type is very similar to the blunt sedge-rocky mtn. fescue/ bearberry community described by Willoughby (1999) and the junegrass-hairy wildrye-brome community described by Corns and Achuff (1982) on steep south-facing slopes in the Subalpine subregion.

\section{Plant COMPOSITION CANopy COVER(\%)}

\section{Mean Range Const.}

\section{SHRUBS}

PRICKLY ROSE

(Rósa acicularis)

$2 \quad 0-3 \quad 75$

BEARBERRY

(Arctostaphylos uva-ursi) $1 \quad 0-5 \quad 25$

FORBS

PLAINS WORMWOOD

(Artemisia campestris) $\quad 1 \quad 0-5 \quad 25$

FRINGED SAGE

$\begin{array}{llll}\text { (Artemisia frigida) } & 7 & 0-17 \quad 75\end{array}$

MOUNTAIN GOLDENROD

$\begin{array}{llll}\text { (Solidago spathulata) } & 1 & 0-5 & 25\end{array}$

LATE YELLOW LOCOWEED

(Oxytropis monticola) $1 \quad 0-3 \quad 25$

GRASSES

JUNEGRASS

(Koeleria macrantha) $19 \quad 13-30 \quad 100$

THREAD-LEAVED SEDGE

$\begin{array}{llll}\text { (Carex filifolia) } & 14 & 0-32 & 50\end{array}$

SEDGE

(Carex spp)

$14 \quad 0-38 \quad 75$

SHEEP FESCUE

(Festuca saximontana) $\quad 1 \quad 0-5 \quad 25$
MOISTURE REGIME:

SUB XERIC

NUTRIENT REGIME:

SUBMESOTROPHIC

ELEVATION:

1560-1720 M (1592 M)

SOIL DRAINAGE:

RAPIDLY

ASPECT:

SOUTH

SLOPE:

$$
25-40 \%
$$

\section{FORAGE PRODUCTION (KG/HA)}

GRASS: 737 (400-1044)

FORBS: 359 (222-495)

SHRUBS: $171(1-400)$

TOTAL: 1147 (800-1378)

\section{SUGGESTED GRAZING CAPACITY}

NON-USE

\section{ENVIRONMENTAL VARIABLES}




\section{UFA10. Bearberry/ Slender wheatgrass \\ (Arctostaphylos uva-ursi/ Agropyron trachycaulum)}

$\mathbf{n}=2$ This community type is found scattered throughout the Upper Foothills subregion on dry, gravelly, well drained river flats. The presence of silverberry, yellow mountain avens, bearberry and early yellow locoweed are very common on these sites.

The poor soil conditions limit the forage productivity and amount of regrowth after grazing. This community type should be rated as secondary or non-use range.

\section{PLANT COMPOSITION CANOPY COVER(\%) \\ Mean Range Const.}

\section{SHRUBS}

SILVERBERRY

(Elaeagnus commutata) $1 \quad 0-1 \quad 50$

YÉLLOW MOUNTAIN AVENS

(Dryas drummondii) $\quad 2 \quad 0-3 \quad 50$

BEARBERRY

(Arctostaphylos uva-ursi) $11 \quad 8-12 \quad 100$

FORBS

STRAWBERRY

(Fragaria virginiana) 22

EARLY YELLOW LOCOWEED

(Oxytropis sericea) 12

YARROW

(Achillea millefolium) 3

GRASSES

JUNEGRASS

(Koeleria macrantha) 3

ROUGH FESCUE

(Festuca scabrella) $\quad 4 \quad 0-8 \quad 50$

SLENDER WHEATGRASS

(Agropyron trachycaulum)6

ALPINE BLUEGRASS

(Poa alpina)

SHEEP FESCUE

(Festuca saximontana)
$12 \quad 0-24 \quad 50$

$1-5 \quad 100$

$0-5 \quad 50$

$0-11 \quad 100$

$5 \quad 0-10 \quad 50$

$2 \quad 0-3 \quad 50$
MOISTURE REGIME:

SUBMESIC

NUTRIENT REGME:

MESOTROPHIC

ELEVATION:

1400-1415 M (1408 M)

SOIL DRAINAGE:

WELL

ASPECT:

SOUTHEAST

SLOPE:

$0-3 \%$

\section{Forage Production (KG/HA)}

GRASS: 400

SHRUBS: 400

TOTAL: 500

SUGgESTEd GRAZING CAPACITY

NON-USE 


\section{UFA11. Fireweed/ Hairy wildrye (Forb meadow) \\ (Epilobium angustifolium/ Elymus innovatus)}

$\mathrm{n}=3$ This community type is found on moist lowland sites adjacent to the lodgepole pine and white spruce dominated forests. It represents the transition from the willow and grass dominated riparian areas to the conifer dominated forests. In the absence of disturbance (fire) it appears that succession of conifers into the grassy meadows shifts the species dominance away from a predominantly graminoid cover to one dominated by forbs such as fireweed, Lindley's aster and palmate leaved coltsfoot. There is also a shift in grass cover away from tufted hairgrass, rough fescue and sedge species to more shade tolerant grass species such as purple oatgrass and hairy wildrye. Periodic burning of this site is required to limit tree and shrub expansion.

This community type is very productive and easily accessible to livestock. It would be rated as primary range.

\section{PLANT COMPOSITION CANOPY COVER(\%) Mean Range Const.}

\section{UNDERSTORY TREES}

WHITE SPRUCE

(Picea glauca)

LODGEPOLE PINE

(Pinus contorta)

SHRUBS

WILLOW

(Salix spp.)

FORBS

FIREWEED

(Epilobium angustifolium)25

STRAWBERRY

(Fragaria virginiana)

LINDLEY'S ASTER

(Aster ciliolatus)

YARROW

(Achillea millefolium) $\quad 7 \quad 3-11 \quad 100$

GRASSES

HAIRY WILDRYE

(Elymus innovatus)

PURPLE OATGRASS

(Schizachne purpurascens)6

TUFTED HAIRGRASS

(Deschampsia cespitosa) 3
$3 \quad 0-8$

67

$6 \quad 0-10 \quad 67$

$16 \cdot 0-25$

67

T-47 100

3-13 100

$0-26 \quad 67$

T-20 100

$0-15 \quad 67$

$0-4 \quad 67$
MOISTURE REGIME:

SUBHYGRIC

NUTRIENT REGIME:

PERMESOTROPHIC

ELEVATION:

1310-1454 M (1401 M)

SOIL DRAINAGE:

MODERATELY WELL

ASPECT:

SOUTHEAST

SLOPE:

$0-2 \%$

Forage ProduCtion (KG/HA)

GRASS: 200

FORBS: 1154

SHRUBS: 400

TOTAL: $1252(1182-1354)$

SUGGESTED GRAZING CAPACITY

$0.7 \mathrm{HA} / \mathrm{AUM}$ OR $1.6 \mathrm{AC} / \mathrm{AUM}$

ENVIRONMENTAL VARIABLES 


\section{UFA12. Rough fescue-Bog sedge \\ (Festuca scabrella-Kobresia myosuroides)}

$\mathrm{n}=2$ This community is very similar to the bog birch/ rough fescue-bog sedge community type described by Willoughby (1999) in the Foothills ecodistrict of the Subalpine subregion. Bog sedge is well adapted to growing on dry alpine slopes and rocky ridges in the mountains. Coms and Achuff (1982), described bog sedge dominated community types on windswept ridges in the alpine subregion of Banff and Jasper National Parks. The sites described in thus community type appear to represent the transition from the Upper Foothills to the Subalpine subregion.

Plant Coyifosition Canopy Cover(\%) mean Range Const.

\section{SHRLBS}

SHRUBBY CTUKTIOLIL

(Potentillafrwictesa) $\quad 1 \quad 0-1 \quad 50$

BEARBERRY

(Arctostaphyios wa-ursa) $6 \quad 0-11 \quad 50$

FORBS

FIREW'EED

(Epilobium chgurtiolium)

OLD MAN'S HHISURS

(Geum trifionm)

FALSE DANDELOY

(Agoseris glowca)

ALPINE HEDYSAR:M

(Hedysarum alpinum)

GRASSES

HAIRY WILDRYE

(Elymus innovarus) $\quad 10 \quad 2-17 \quad 100$

ROUGH FESCUE

$\begin{array}{llll}\text { (Festuca scabrella) } & 38 & 32-43 & 100\end{array}$

BOG SEDGE

(Kobresia myosuroides) $25 \quad 12-37 \quad 100$

SLENDER WHEATGRASS

(Agropyron trachycaulum) $18 \quad 12-22 \quad 100$

SEDGE

(Carex spp.)
$19 \quad 15-21 \quad 100$

$3 \quad 1-6 \quad 100$

$0-5 \quad 22$

2-13 100

\section{ENVIRONMENTAL VARIABLES}

MOISTURE REGIME:

MESIC

NUTRIENT REGIME:

MESOTROPHIC

ELEVATION:

$1432-1828 \mathrm{M}(1688 \mathrm{M})$

SOIL DRANAGE:

WELL

ASPECT:

SOUTHERLY

SLOPE:

$0-40 \%$

\section{FORAGE PRODUCTION (KG/HA)}

GRASS: $966(832-1234)$

FORBS: $149(98-202)$

TOTAL: $1115(932-1434)$

\section{SUGGESTED GRAZING CAPACITY} 0.8 HAUM 


\section{UFA13. Arctic rough fescue \\ (Festuca altaica)}

$\mathbf{n = 2} \quad$ This community was described at higher elevations in Willmore Wilderness Park. Bork(1994), described this community type on alpine and subalpine slopes where climate and soil conditions are still suitable for fescue to dominate in the stand. The community has a subhygric moisture regime and is moderately well drained. Forb species such as globeflower, fleabane, monkshood, and mountain heliotrope are all characteristic of these high elevation meadows.

This community is much wetter than the rough fescue-bog sedge community (UFA12) previously described and is similar to the forb meadows community type described by Willoughby (1999) in the Subalpine subregion.

\section{PLANT COMPOSITION CANOPY COVER(\%) Mean Range Const.}

SHRUBS

DWARF BILBERRY

(Vaccinium caespitosum) $3 \quad 2-3 \quad 100$

DWARF BIRCH

$\begin{array}{llll}\text { (Betula glandulosa) } & 1 & 0-1 & 50\end{array}$

FORBS

YARROW

(Achillea millefolium) $2 \quad 1-3 \quad 100$

MONKSHOOD

(Aconitum delphinifolium) $1 \quad 0-2 \quad 100$

MOUNTAIN HELIOTROPE

$\begin{array}{llll}\text { (Valeriana sitchensis) } & 1 & 0-2 & 100\end{array}$

WANDERING DAISY

(Erigeron peregrinus) $2 \quad 0-3 \quad 50$

GLOBEFLOWER

(Trollius albiflorus) $\quad 2 \quad 0-4 \quad 50$

GRASSES

ROUGH FESCUE

(Festuca altaica)

MOUNTAIN TIMOTHY

(Phleum commutatum)

SLENDER WHEATGRASS

(Agropyron trachycaulum)2

SEDGE

(Carex spp.)

TUFTED HAIRGRASS

(Deschampsia cespitosa)
MOISTURE REGIME:

SUBHYRGIC

NUTRIENT REGIME:

PERMESOTROPHIC

ELEVATION:

$1510-2000 \mathrm{M}(1755 \mathrm{M})$

SOIL DRAINAGE:

MODERATELY WELL

FORAGE PRODUCTION (KG/HA)

GRASS: 743 (527-959)

FORBS: 372 (368-375)

TOTAL: $1115(895-1334)$

$0.8 \mathrm{HA} / A U M$ OR 1.7 AC/AUM

\section{ENVIRONMENTAL VARIABLES}




\section{UFA14. Cow parsnip-Veiny meadow rue/ Fringed brome (Heracleum lanatum-Thalictrum venulosum/Bromus ciliatus)}

$\mathrm{n}=1 \quad$ This community type is transitional between the Lower Foothills and Upper Foothills subregions. It was described on fine textured, silty soils adjacent to the Baptiste river west of Rocky Mountain House. Grazed stands of this community type were also described in the Solomon valley, west of Hinton. Increased grazing pressure generally allows timothy, Kentucky bluegrass and dandelion to increase with a corresponding drop in the cover of cow parsnip, meadow rue and the native grasses and sedges. The high moisture and nutrient regime of this.site makes it extremely productive, and once it has been invaded by agronomic species it is highly palatable for domestic livestock. It is difficult to find representative stands of this community type that have not been grazed.

\section{PLANT COMPOSITION CANOPY COVER(\%)} MEAN RANGE CONST.

$\begin{array}{llll}\begin{array}{l}\text { SHRUBS } \\ \text { WILLOW }\end{array} & & \\ \text { (Salix spp.) } & & & \\ \begin{array}{l}\text { SNOWBERRY } \\ \text { (Symphoricarpos } \\ \quad \text { occidentalis) }\end{array} & 4 & - & 100 \\ \begin{array}{l}\text { FORBS } \\ \text { COW PARSNIP }\end{array} & & & \\ \begin{array}{l}\text { (Heracleum lanatum) } \\ \text { VEINY MEADOW RUE }\end{array} & 21 & - & 100 \\ \begin{array}{l}\text { (Thalictrum venulosum) } \\ \text { TALL LUNGWORT }\end{array} & 10 & - & 100 \\ \begin{array}{l}\text { (Mertensia paniculata) } \\ \text { TALL LARKSPUR }\end{array} & 11 & - & 100 \\ \text { (Delphinium glaucum) } & 8 & - & 100 \\ \begin{array}{l}\text { FIREWEED } \\ \text { (Epilobium angustifolium)5 }\end{array} & - & 100 \\ \begin{array}{l}\text { AMERICAN VETCH } \\ \text { (Vicia americana) }\end{array} & 3 & - & 100 \\ \begin{array}{l}\text { GRASSES } \\ \text { KENTUCKY BLUEGRASS } \\ \text { (Poa pratensis) }\end{array} & 15 & - & 100 \\ \begin{array}{l}\text { AWNED SEDGE } \\ \text { (Carex atherodes) }\end{array} & 7 & - & 100 \\ \begin{array}{l}\text { SLENDER WHEATGRASS } \\ \text { (Agropyron trachycaulum) } 4\end{array} & - & 100 \\ \begin{array}{l}\text { SEDGE } \\ \text { (Carex spp.) }\end{array} & 12 & - & 100 \\ \begin{array}{l}\text { FRINGED BROME } \\ \text { (Bromus ciliatus) }\end{array} & 6 & - & 100\end{array}$

\section{ENVIRONMENTAL VARIABLES}

MOISTURE REGIME: SUBHYGRIC

NUTREENT REGIME: PERMESOTROPHIC

ELEVATION: $1060 \mathrm{M}$

SOIL DRAINAGE: MODERATELY WELL

Forage Production (KG/HA)

TOTAL: 5000

\section{SUGGESTED GRAZING CAPACITY}

$0.3 \mathrm{HA} / A U M$ OR $0.4 \mathrm{AC} / \mathrm{AUM}$ 


\section{UFA15. Hairy wildrye-Sedge (Elymus innovatus-Carex spp.)}

$\mathrm{n}=1$ This community type was described on south facing slopes along Wilson Creek in the Upper Foothills subregion. Corns and Achuff (1982) described similar community types in the subalpine of Banff and Jasper National Parks. These included the shrubby cinquefoil/ hairy wildrye and hairy wildrye/ bearberry-juniper community types. Both of these community types were associated with steep south facing slopes. The presence of this community type may indicate the transition to the Subalpine subregion.

This community type does not produce a large amount of forage because of the dry site conditions and poor nutrient content of the soil, but the lack of open areas for livestock grazing in this subregion makes these grassland sites attractive to livestock.

\section{Plant COMPOSITION CANOPY COVER(\%)} mean Range Const.

FOQRBS

SHOWY LOCOWEED

(Oxytropis splendens)

AMERICAN VETCH

(Vicia americana)

LOW GOLDENROD

(Solidago multiradiata)

STRAWBERRY

$\begin{array}{llll}\text { (Fragaria virginiana) } & 2 & - & 100\end{array}$

FIREWEED

(Epilobium angustifolium)

ALPINE HEDYSARUM

(Hedysarum alpinum)

GRASSES

HAIRY WILDRYE

(Elymus innovatus)

ARCTIC BLUEGRASS

(Poa arctica)

SEDGE

(Carex spp.)

$\begin{array}{lll}18 & - & 100 \\ 8 & - & 100 \\ 6 & - & 100 \\ 2 & - & 100 \\ 4 & - & 100 \\ 1 & - & 100 \\ & & \\ 50 & - & 100 \\ 6 & - & 100 \\ 5 & - & 100\end{array}$

\section{ENVIRONMENTAL VARIABLES}

MOISTURE REGIME:

SUBMESIC

NUTRIENT REGIME:

SUBMESOTROPHIC

ELEVATION:

$1860 \mathrm{M}$

SOIL DRAINAGE:

WELL

ASPECT:

SOUTHWEST

SLOPE:

$5 \%$

\section{FORAGE PRODUCTION(KG/HA)}

GRASS: 222

FORBS: 66

SHRUBS: 8

TOTAL: 296
SUGGESTED GRAZING CAPACITY

$3.1 \mathrm{HANAUM}$ OR 6.9 AC/AUM 


\section{UFA16. Hairy wildrye-Rough fescue/ Bearberry \\ (Elymus innovatus-Festuca scabrella/ Arctostaphylos uva-ursi)}

$\mathrm{n}=1$ This community was described in the Ghost area west of Calgary on a well drained, level valley floor. It appears to represent a grazed rough fescue, bearberry or hairy wildrye dominated community. Willoughby (2000) found that heavy grazing on the rough fescue dominated grasslands often leads to a community that is dominated by sedge and hairy wildrye. Protection from grazing or a reduction in stocking rate allows this community type to recover back to a rough fescue dominated community. The time frame for complete recovery takes 20 years (Willoughby 2000)

\section{Plant COMPOSITION CANOPY COVER(\%) MEAN RANGe CONST.}

\begin{tabular}{|c|c|c|c|}
\hline \multicolumn{4}{|l|}{ SHRUBS } \\
\hline \multicolumn{4}{|l|}{ BEARBERRY } \\
\hline (Arctostaphylos uva-ursi) & 7 & - & 100 \\
\hline SHRUBBY CINQUEFOIL & 1 & & 100 \\
\hline \multicolumn{4}{|l|}{ FORBS } \\
\hline \multicolumn{4}{|l|}{ FIREWEED } \\
\hline \multicolumn{4}{|l|}{ (Epilobium angustifolium)5 } \\
\hline \multicolumn{4}{|l|}{ GRACEFUL CINQUEFOIL } \\
\hline \multicolumn{4}{|l|}{ GOLDENROD } \\
\hline (Solidago missouriensis) & 1 & - & 100 \\
\hline \multicolumn{4}{|l|}{ SMOOTH ASTER } \\
\hline \multicolumn{4}{|l|}{ STRAWBERRY } \\
\hline (Fragaria virginiana) & 13 & - & 100 \\
\hline \multicolumn{4}{|l|}{ VENY MEADOW RUE } \\
\hline \multicolumn{4}{|l|}{ GRASSES } \\
\hline \multicolumn{4}{|l|}{ SLENDER WHEATGRASS } \\
\hline (Agropyron trachycaulum) & & - & 100 \\
\hline \multicolumn{4}{|l|}{ HAIRY WILDRYE } \\
\hline (Elymus innovatus) & 11 & - & 100 \\
\hline \multicolumn{4}{|l|}{ SEDGE } \\
\hline (Carex spp.) & 1 & - & 100 \\
\hline $\begin{array}{l}\text { ROUGH FESCUE } \\
\text { (Festuca scabrella) }\end{array}$ & 2 & - & 100 \\
\hline & & 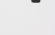 & \\
\hline
\end{tabular}

\section{ENVIRONMENTAL VARIABLES}

MOISTURE REGIME:

MESIC

NUTRIENT REGIME:

MESOTROPHIC

ELEVATION:

1680

SOIL DRAINAGE:

MODERATELY WELL

ASPECT:

EAST

SLOPE:

$3 \%$

\section{FORAGE PRODUCTION(KG/HA)}

GRASS: 2008

FORBS: 557

TOTAL: 2121

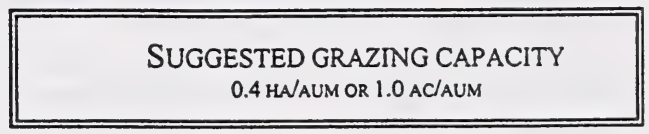




\section{UFA17. Idaho fescue-Parry oatgrass-Sedge \\ (Festuca idahoensis-Danthonia parryi-Carex spp.)}

$\mathbf{n}=2$ This community type was described in the Ghost area west of Calgary. This area represents a transition between the Montane and Upper Foothills subregions. This community type is very similar to moderately and heavily grazed rough fescue dominated communities in the Montane subregion. Both Idaho fescue and Parry oatgrass are more characteristic of the Montane subregion and increase with increased grazing pressure. Protection from grazing will often allow this community type to recover back to a Rough fescue-Parry oatgrass dominated community type.

\section{PLANT COMPOSITION CANOPY COVER(\%)}

SHRUBS

MEAN Range Const.

SHRUBBY CINQUEFOIL

$\begin{array}{llll}\text { (Potentilla fruticosa) } & 3 & 2-3 & 100\end{array}$

BOǴ BIRCH

$\begin{array}{llll}\text { (Betula glandulosa) } & 3 & 0-5 & 50\end{array}$

WILLOW

(Salix spp)

$2 \quad 0-4 \quad 50$

FORBS

STRAWBERRY

(Fragaria virginiana)

YARROW

(Achillea millefolium)

VEINY MEADOW RUE

(Thalictrum venulosum)

(Potentilla gracilis)

GRASSES

SEDGE

(Carex spp.)

IDAHO FESCUE

(Festuca idahoensis)

PARRY'S OATGRASS

(Danthonia parryi)

HAIRY WILDRYE

(Elymus innovatus)

$22 \quad 10-33 \quad 100$

$13 \quad 6-18 \quad 100$

$10 \quad 7-11 \quad 100$

$4 \quad 0-8 \quad 50$

$39 \quad 28-50 \quad 100$

$21 \quad 16-25 \quad 100$

$2 \quad 0-3 \quad 50$

$22 \quad 28-50 \quad 100$

\section{ENVIRONMENTAL VARIABLES}

MOISTURE REGIME:

SUBHYGRIC

NUTRIENT REGIME:

MESOTROPHIC

ELEVATION:

$1400 \mathrm{M}$

SOIL DRAINAGE:

IMPERFECTLY

ASPECT:

SOUTH

SLOPE:

$2-15 \%$

FORAGE PRODUCTION(KG/HA)

TOTAL: 1467 *ESTIMATE

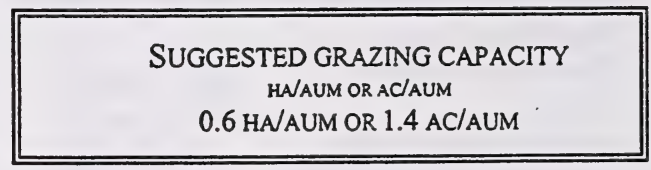




\section{UFB1. Willow-Bog birch/ Water sedge (Salix spp.-Betula glandulosa/ Carex aquatilis)}

$\mathrm{n}=45$ This shrub community appears in areas with very poor drainage. It is found in association with the wetter water sedge meadows (UFA1). These sites are fairly productive but are difficult to graze due to the moist ground conditions and heavy shrub cover which reduces access and mobility in the area. Increased flooding and prolonged water logging may result in the disappearance of willow and a transition to a water sedge meadow.

PLANT COMPOSITION CANOPY COVER(\%) MEAN RANGe CONST.

\section{SHRUBS}

\section{BARCLAY'S WILLOW}

(Salix barclayi).

Wịlow

(Salix spp.)

BOG BIRCH

(Betula glandulosa)

FORBS

ARROW LEAVED COLTSFOOT

(Petasites sagittatus) 1

LINDLEY'S ASTER

(Aster ciliolatus)

STICKY PURPLE GERANIUM

(Geranium viscosissimum)1

GRACEFUL CINQUEFOIL

(Potentilla gracilis)

ARCTIC ASTER

(Aster sibiricus)

GRASSES

WATER SEDGE

(Carex aquatilis)

SEDGE

(Carex spp.)

TUFTED HAIRGRASS

(Deschampsia cespitosa) 6

MARSH REEDGRASS

(Calamagrostis canadensis
$2 \quad 0-48 \quad 9$

$28 \quad 0-65 \quad 84$

$11 \quad 0-58 \quad 56$

$0-13 \quad 36$

$0-6 \quad 11$

$0-14 \quad 11$

$0-7 \quad 29$

$1 \quad 0-8 \quad 2$

$11 \quad 0-76 \quad 24$

$40 \quad 0-82 \quad 71$

$0-35 \quad 80$

$0-13 \quad 11$

\section{ENVIRONMENTAL VARIABLES}

MOISTURE REGIME :

SUBHYDRIC

NUTRIENT REGIME

MESOTROPHIC

ELEVATION:

SOIL DRAINAGE:

$1227-1820 \mathrm{M}(1443 \mathrm{M})$

POORLY

ASPECT:

VARIABLE

SLOPE:

$0-14 \%$

\section{Forage PRODUCTION (KG/HA)}

GRASS: 1325 (340-3000)

FORBS: $126(2-402)$

SHRUBS: 732 (54-2180)

TOTAL: 2105 (814-4662)

SUGGESTED GRAZING CAPACITY NON-USE 


\section{UFB2. Willow/ Slender wheatgrass-Sedge (Salix spp./ Agropyron trachycaulum-Carex spp.)}

$\mathrm{n}=4$ This community type is very similar to the tufted hairgrass-sedge-slender wheatgrass c.t. (UFA4) previously described. Both community types appear to represent the various stages of succession onto tufted hairgrass meadows. When these communities are protected from disturbance (fire and grazing), willow and bog birch expand and tufted hairgrass declines. Willow growth also appears to favour the growth of tall forbs (veiny meadow rue, fireweed, aster) and slender wheatgrass. Fire has played a dominant role in controlling brush encroachment in the past and continued protection will allow continued shrub expansion, resulting in a decline in forage production.

Plant Composition Canopy Cover $(\%)$ MEAN RANGe CONST.

SHRUBS

BARCLAY'S WILLOW

(Salix barclayi)

BOG BIRCH

(Betula glandulosa)

FORBS

VENNY MEADOW RUE

(Thalictrum venulosum)

OLD MAN'S WHISKERS

(Geum triflorum)

STRAWBERRY

(Fragaria virginiana)

TALL LARKSPUR

(Delphinium glaucum)

LINDLEY'S ASTER

(Aster ciliolatus)

YARROW

(Achillea millefolium)

GRASSES

GRACEFUL SEDGE

(Carex praegracilis)

SEDGE

(Carex spp.)

13

$0-19 \quad 75$

$13 \quad 2-23 \quad 100$

$\begin{array}{lll}7 & 3-9 & 100\end{array}$

$17 \quad 3-23 \quad 100$

$13 \quad 10-15 \quad 100$

$1 \quad 0-\mathrm{T} \quad 50$

$10 \quad 0-26 \quad 75$

$8 \quad 4-13 \quad 100$

SLENDER WHEATGRASS

(Agropyron trachycaulum) $15 \quad 0-22 \quad 75$

CALIFORNIA OATGRASS

(Danthonia californica) 7

TUFTED HAIRGRASS

(Deschampsia cespitosa) 4 $\begin{array}{lll}24 & 0-37 \quad 75\end{array}$

$7 \quad 0-29 \quad 25$

$0-20 \quad 100$

$0-12 \quad 75$
ENVIRONMENTAL VARIABLES

MOISTURE REGIME:

SUBHYGRIC

NUTRIENT REGIME :

PERMESOTROPHIC

ELEVATION:

SOIL DRAINAGE:

1349-1615 M (1455 M)

WELL

ASPECT:

NORTH TO NORTHEAST

SLOPE:

$0-4 \%$

FORAGE PRODUCTION (KG/HA)

GRASS: 1573

FORBS: 735

TOTAL: $1669(900-2308)$

SUGGESTED GRAZING CAPACITY

0.5 HANAUM OR 1.2 ACRES/AUM 


\section{UFB3. Willow/ Tufted hairgrass \\ (Salix barclayi/ Deschampsia cespitosa)}

$\mathrm{n}=16$ This community type is found in association with the tufted hairgrass-sedge c.t. (UFA3). Willow encroachment into a tufted hairgrass meadow eventually results in this community type. Historically, fire has played an important role in the maintenance of the grassland community types in this subregion. Continued fire suppression will eventually allow willow and bog birch to invade many of these grassy meadows.

The encroachment of willow onto the tufted hairgrass-sedge c.t. causes a decline in forage production from $2200 \mathrm{~kg} / \mathrm{ha}$ to $1200 \mathrm{~kg} / \mathrm{ha}$ for grass and forb production. Continued protection of this community type from disturbance will most likely lead to the development of a community type similar to the willow/ slender wheatgrass (UFB2) and then to the pussy willow shrubland (UFB7). The latter community has a high cover of willow (71\%) and very little forage for domestic livestock.

Plant COMPOSITION CANopy Cover(\%) ..

SHRUBS

BARCLAY'S WILLOW

(Salix barcalyi)

BOG BIRCH

(Betula glandulosa)

FORBS

YARROW

$\begin{array}{llll}\text { (Achillea millefolium) } & 6 & 2-14 & 100\end{array}$

WILD STRAWBERRY

$\begin{array}{llll}\text { (Fragaria virginiana) } & 9 & 0-30 & 94\end{array}$

LINDLEY'S ASTER

(Aster ciliolatus)

VENNY MEADOW RUE

(Thalictrum venulosum)

GRACEFUL CINQUEFOIL

(Potentilla gracilis)

DANDELION

(Taraxacum offincinale)

GRASSES

TUFTED HAIRGRASS

(Deschampsia cespitosa) $18 \quad 0-38 \quad 100$

SLENDER WHEATGRASS

(Agropyron trachycaulum)8

GRACEFUL SEDGE

(Carex praegracilis)

Mean Range Const.

$\begin{array}{lll}33 & 0-85 & 88\end{array}$

$\begin{array}{lll}11 & 0-75 \quad 88\end{array}$

$11 \quad 0-25 \quad 81$

$0-21 \quad 81$

$0-10 \quad 88$

$0-11 \quad 50$

PURPLE OATGRASS

(Schizachne purpurascens) 4

$0-25 \quad 94$

$0-31 \quad 69$

$0-32 \quad 56$
ENVIRONMENTAL VARIABLES

MOISTURE REGIME:

SUBHYGRIC

NUTRIENT REGIME:

ELEVATION:

PERMESOTROPHIC

SOIL DRAINAGE:

MODERATELY WELL

ASPECT:

VARIABLE

SLOPE:

$0-10 \%$

FORAGE PRODUCTION(KG/HA)

GRASS: 1168 (275-2484)

FORBS: 405 (8-1052)

SHRUBS: 772 (36-2250)

TOTAL: 2058 (500-5132)

SUGGESTED GRAZING CAPACITY

0.4 HAVAM OR 1.0 ACRES/AUM 


\section{UFB4. Willow/ Rough fescue \\ (Salix barclayi/ Festuca scabrella)}

$\mathrm{n}=2$ This community type was described by Bork (1994) in Willmore Wilderness Park. Bork felt this community type originated from recent shrub encroachment onto rough fescue grasslands. Continued shrub expansion will result in decreasing forage productivity. Bork also felt that fescue will be replaced by wheatgrass and sedge plant species. These plants being better adapted to shading and competition from adjacent shrubs.

PLANT COMPOSITION CANOPY COVER(\%) MEAN RANGE CONST.

TREES

SUBALPINE FIR -

(Ábies lasiocarpa)

SHRUBS

$1 \quad 0-1 \quad 50$

WILLOW

(SALIX SPP)

$26 \quad 25-27 \quad 100$

BOG BIRCH

$24 \quad 10-38 \quad 100$

YELLOW MOUNTAIN AVENS

(Dryas drummondii)

ALPINE BEARBERRY

(Arctostaphylos rubra) $\quad 4 \quad 0-7 \quad 50$

FORBS

ALPINE BISTORT

$\begin{array}{llll}\text { (Polygonum viviparum) } & 10 & \text { T-19 } & 100\end{array}$

MONKSHOOD

(Aconitium delphinifolium) $3 \quad 0-5 \quad 50$

INDIAN PAINTBRUSH

(Castelleja miniata)

$2 \quad 0-4 \quad 50$

GRASSES

ROUGH FESCUE

$\begin{array}{llll}\text { (Festuca scabrella) } \quad 13 \quad 12-14 & 100\end{array}$

GRACEFUL SEDGE

(Carex praegracilis)
$2 \quad 0-3 \quad 50$
ENVIRONMENTAL VARIABLES

MOISTURE REGIME:

HYGRIC

NUTRIENT REGIME:

PERMESOTROPHIC

ELEVATION:

$1465-1560 \mathrm{M}(1528 \mathrm{M})$

SOIL DRAINAGE:

MODERATELY WELL

ASPECT:

WESTERLY

SLOPE:

$0-10 \%$

FORAGE PRODUCTION(KG/HA)

GRASS: 640 (600-680)

FORBS: $132(64-200)$

SHRUBS: 733 (150-1316)

TOTAL: $1505(950-2060)$

SUGGESTED GRAZING CAPACITY

0.6 HANAUM OR 1.3 ACRES/AUM 


\section{UFB5. Bog birch/ Rough fescue/ Bearberry \\ (Betula glandulosa/ Festuca scabrella/ Arctostaphylos uva-ursi)}

$\mathrm{n}=19$ This community type is very similar to the rough fescue-bearberry (UFA7) type previously described, but it is successionally more advanced. The soils on this community type are gravelly, drier and have a poorer nutrient regime than the other rough fescue and tufted hairgrass dominated community types. The lack of fire on this community type has allowed the shrub cover to expand, reducing forage productivity for wildlife and domestic livestock. In one study, burning a bog birch/ rough fescue/ bearberry community type twice in 3 year intervals controlled birch growth and increased total forage production by over $40 \%$ compared to the unburned control (Bork, 1990).

Plant COMPOSITION Canopy Cover(\%) MEAN RANGe CONST.

\section{SHRUBS}

BOG BIRCH

(Betula glandulosa)

WILLOW

(Salix spp)

BEARBERRY

(Arctostaphylos uva-ursi) 9

$33 \quad 1-60$

100

FORBS

LINDLEY'S ASTER

(Aster ciliolatus)

ALPINE MILKVETCH

(Astragalus alpinus)

WILD STRAWBERRY

(Fragaria virginiana)

FIREWEED

(Epilobium angustifolium) 3

SLENDER BLUE BEARDTONGUE

(Penstemon procerus)

OLD MAN'S WHISKERS

(Geum triflorum)

$\begin{array}{lll}1 & 0-8 & 39\end{array}$

GRASSES

ROUGH FESCUE

(Festuca scabrella)

GRACEFUL SEDGE

(Carex praegracilis)

$4 \quad 0-17 \quad 89$

SLENDER WHEATGRASS

(Agropyron trachycaulum) 3

CALIFORNIA OATGRASS

(Danthonia californica)
ENVIRONMENTAL VARIABLES

MOISTURE REGIME:

SUBHYGRC

NUTRIENT REGIME:

PERMESOTROPHIC

ELEVATION:

SOIL DRAINAGE:

1303-1798 M (1562 M)

WELL

ASPECT:

SLOPE:

SOUTHERLY

$1-25 \%$

FORAGE PRODUCTION(KG/HA)

GRASS: $1206(856-1728)$

FORBS: 173 (14-394)

SHRUBS: $306(140-582)$

TOTAL: 1523 (1000-1986)

\section{SUGGESTED GRAZING CAPACITY}

0.6 HAAUM OR 1.3 ACRES/AUM 


\section{UFB6. Willow/ California oatgrass-Sedge (Salix barclayi/ Danthonia californica-Carex spp.)}

$\mathrm{n}=4$ This community type likely develops from willow encroachment onto an oatgrass dominated meadow. The oatgrass meadows are found on dry, gravelly soils. These meadows may also form in frost pockets. The spread of willow is likely caused by the lack of natural disturbance, such as fire. The cover of willow on this community type is fairly extensive. This will restrict access of domestic livestock. This community type would be rated as secondary range.

\section{Plant COMPOSITION CANOpy COVER(\%)} MEAN Range Const.

\section{SHRUBS}

WILLOW SPP.

(Salix barclayi)

$20 \quad 0-60 \quad 50$

BOG BIRCH

(Betula glandulosa)

FORBS

YARROW

(Achillea millefolium) 10

GRACEFUL CINQUEFOIL

(Potentilla gracilis)

WILD STRAWBERRY

(Fragaria virginiana) 20

SLENDER BLUE BEARDTONGUE

(Penstemon procerus) 2

ALSIKE CLOVER

(Trifolium pratense) $\quad 4 \quad 0-17 \quad 25$

FIREWEED

(Epilobium angustifolium)2 $\quad 0-4 \quad 100$

VEINY MEADOW RUE

(Thalictrum venulosum) 4

GRASSES

CALIFORNIA OATGRASS

(Dainthonia californica) $33 \quad 19-56 \quad 100$

GRACEFUL SEDGE

(Carex praegracilis) $\quad 10 \quad 0-30 \quad 50$

SHEEP FESCUE

(Festuca saximontana) $7 \quad 0-22 \quad 50$

SLENDER WHEATGRASS

(Agropyron trachycaulum)

\section{ENVIRONMENTAL VARIABLES}

MOISTURE REGIME:

SUBHYGRIC

NUTRIENT REGIME:

PERMESOTROPHIC

ELEVATION:

$1400-1500 \mathrm{M}(1458 \mathrm{M})$

SOIL DRAINAGE:

MODERATELY WELL

ASPECT:

WEST

SLOPE:

$0-30 \%$

FORAGE PRODUCTION(KG/HA)

GRASS: 1324 (598-2050)

FORBS: $400(382-418)$

SHRUBS: 300

TOTAL: $1874(1316-2432)$

\section{SUGGESTED GRAZING CAPACITY}

0.5 HAAUM OR 1.1 ACRES/AUM 


\section{UFB7. Pussy willow shrubland \\ (Salix discolor)}

$\mathbf{n}=\mathbf{2}$ This community type is common along riparian areas, swamps and fringes of marshes and lakes. It appears to be successionally more advanced than the other willow dominated community types described in this guide. As the willow cover expands over time it shades the understory vegetation resulting in a loss of forage productivity. This community type produces only $200 \mathrm{~kg} / \mathrm{ha}$ and is generally inaccessible to domestic livestock. This community type should be rated as non-use.

\section{PLANT COMPOSITION CANOPY COVER(\%) MEAN RANGE CONST.}

\section{SHRLBS}

PUSSY WILLO H

(Salix discolo.

BÖ BIRCH

(Betula giuncisings) $\quad 20 \quad 8-30 \quad 100$

FORBS

LNDDLEY'S ASTER

(Aster cillolians)

PALMATE LEAI D COLTSFOOT

(Petasites fuimaitus) 3

WILD STRAWHERRY

(Fragarla virgimiana)

GRASSES

TUFTED HAIRCRASS

(Deschampsia cespioosa) 5

MARSH REEDGRASS

(Calamagros:us

canadensis)
$71 \quad 70-71 \quad 100$

$5-7 \quad 100$

$0-0 \quad 100$

$1-7 \quad 100$

$1-9 \quad 100$

$3 \quad 0-5 \quad 100$
ENVIRONMENTAL VARIABLES

MOISTURE REGIME:

SUBHYGRIC

NUTRIENT REGIME:

PERMESOTROPHIC

ELEVATION:

$1318-1325 \mathrm{M}(1322 \mathrm{M})$

SOIL DRAINAGE:

MODERATELY WELL

FORAGE PRODUCTION(KG/HA)

TOTAL: $181(101-261)$

\section{SUGGESTED GRAZING CAPACITY}

NON-USE 


\section{UFB8. Willow/ Hairy wildrye-Sedge \\ (Salix barclayi/Elymus innovatus-Carex spp.)}

$\mathrm{n}=2$ This plant community represents a rough fescue-hairy wildrye community type (UFA6) that has continued to undergo succession in the absence of fire and grazing. Willow cover has increased, shading the growth of grasses (rough fescue) and allowing tall-growing forbs (fireweed, aster, veiny meadow rue) to increase. Continued protection from disturbance will allow succession to shrub and eventually tree species. The understorey vegetation will be increasingly shaded and forage production will continue to decrease.

Plant COMPOSITION CANOPY COVER(\%) MEAN RANGE CONST.

TREES

WHITE SPRUCE.

(Picea glauca)

SHRUBS

WILLOW SPP.

(Salix barclayi)

BOG BIRCH

$\begin{array}{llll}\text { (Betula glandulosa) } & 21 & 5-36 \quad 100\end{array}$

FORBS

LNDLEY'S ASTER

(Aster ciliolatus)

VEINY MEADOW RUE

(Thalictrum venulosum)

FIREWEED

(Epilobium angustifolium) 5

STRAWBERRY

$\begin{array}{llll}\text { (Fragaria virginiana) } & 11 & 8-13 \quad 100\end{array}$

TALL LUNGWORT

(Mertensia paniculata) $4 \quad 0-6 \quad 100$

GRASSES

GRACEFUL SEDGE

(Carex praegracilis) $\quad 32 \quad 20-44 \quad 100$

HAIRY WILDRYE

(Elymus innovatus)

PURPLE OATGRASS

(Schizachne purpurascens) 22

SLENDER WHEATGRASS

(Agropyron trachycaulum) 22

\section{ENVIRONMENTAL VARIABLES}

MOISTURE REGIME:

SUBHYGRIC

NUTRIENT REGIME:

PERMESOTROPHIC

ELEVATION:

1371-1400 M (1386) M

SOIL DRAINAGE:

MODERATELY WELL

ASPECT:

NORTHEAST

SLOPE:

$0-3 \%$

FORAGE PRODUCTION(KG/HA)

TOTAL: $1550(900-2200)$

SUGGESTED GRAZING CAPACITY

0.5 HA/AUM OR 1.1 ACRES/AUM 


\section{UFB9. Bog birch/ Sedge-Marsh reedgrass (Betula glandulosa/ Carex spp.-Calamagrostis canadensis)}

$\mathrm{n}=1$ This community type occupies valley drainages on soils that are saturated with water for part of the growing season. This type is very similar to the willow-bog birch/ sedge (UFB1) c.t, but lacks the willow cover. It is not clear why there is no willow cover on this type. It is possible that bog birch is better adapted to growing on poor nutrient soils. The presence of marsh reedgrass may indicate the transition from the Lower Foothills to Upper Foothills subregion. Willoughby (1992), observed that marsh reedgrass was more abundant on these lowland sites at lower elevations.

The thick cover of bog birch and very wet conditions restrict access to domestic livestock. Consequently, this community type would be rated as secondary or non-use range.

\section{Plant COMPOSITION CANopy COVER(\%) MEAN RANGe CONST.}

\section{SHRUBS}

BOG BIRCH

(Betula glandulosa)

FORBS

DWARF RASPBERRY

(Rubus arcticus)

GRASSES

MARSH REEDGRASS

(Calamagrostis canadensis) 11

TUFTED HAIRGRASS

(Deschampsia cespitosa)

BROWNISH SEDGE

(Carex brunnescens)

$\begin{array}{lll}39 & - & 100 \\ 1 & - & 100 \\ 11 & - & 100 \\ 2 & - & 100 \\ 11 & - & 100\end{array}$

\section{ENVIRONMENTAL VARIABLES}

MOISTURE REGIME:

HYGRIC

NUTRIENT REGIME:

SUBMESOTROPHIC

ELEVATION:

$1513 \mathrm{M}$

SOIL DRAINAGE:

IMPERFECTLY

ASPECT:

WEST

SLOPE:

. $3 \%$

\section{FORAGE PRODUCTION(KG/HA)}

GRASS: 796

FORBS: 58

SHRUBS: 322

TOTAL: 1176

\section{SUGGESTED GRAZING CAPACITY \\ NON-USE}




\section{UFB10. Willow-Bog birch/ Sedge (Salix barclayi-Betula glandulosa/Carex spp.)}

$\mathrm{n}=12$ This type is very similar to the willow-bog birch/ water sedge community type (UFB1), but the soils are drier and betrer drained. The drier soil conditions favour the growth of graceful sedge over water sedge.

This community type has a thick cover of bog birch and willow which restricts livestock access to the forage. This community type would be rated as secondary or non-use range.

Plant CONIPOSITION Canopy COVER(\%) MEAN RaNGe CONST.

\section{SHRL'BS}

BOG BIRCH

(Betula glansidiosa)

Wiulow

(Sälix spp)

FORBS

LNDLEY'S AS'ER

(Aster chibloins)

YARROW

(Achillea mulierolium)

STRAWBERRY

(Fragarionggimana)

TALLLUNGHORT

(Mertensia piniculata) $2 \quad 0-6 \quad 58$

VENY MEADOW RLE

$\begin{array}{llll}\text { (Thalictrum venu!osum) } & 3 & 0-8 & 67\end{array}$

GRASSES

GRACEFUL SEDCE

(Carex praegracilus)

$21 \quad 0-53 \quad 67$

SLENDER WHEATGRASS

(Agropyron trachycaulum)

HAIRY WILDRYE

(Elymus innovatus)

TUFTED HAIRGRASS

(Deschampsia cespitosa) 3
$21 \quad 0-55 \quad 75$

$33 \quad 2-46 \quad 100$

$4 \quad 0-15 \quad 58$

$4 \quad 0-11 \quad 92$

$4 \quad 0-14 \quad 67$

$4 \quad 0-27 \quad 58$

$4 \quad 0-25 \quad 50$

$0-10 \quad 67$

\section{ENVIRONMENTAL VARIABLES}

MOISTURE REGIME:

HYGRIC

NUTRENT REGIME: MESOTROPHIC

ELEVATION:

1356-1646 M (1523 M)

SOIL DRAINAGE:

IMPERFECTLY

ASPECT:

VARIABLE

SLOPE:

$0-15 \%$

\section{FORAGE PRODUCTION(KG/HA)}

GRASS: 937 (500-1416)

FORBS: $176(10-568)$

SHRUB: 730 (200-1522)

TOTAL: $1730(800-2468)$

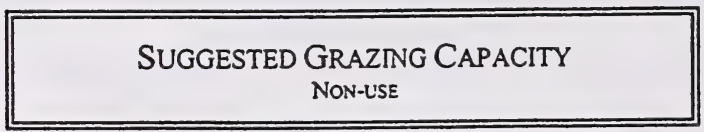




\section{UFB11. Willow-Bog birch \\ (Salix barclayi-Betula glandulosa)}

$\mathbf{n = 1 1}$ This community type is very similar to the willow-bog birch/ sedge c.t. (UFB1), but is successionally more advanced. The lack of fire has allowed continued expansion of the shrub cover. This has restricted access to livestock and lowered forage productivity. This community type would be rated as non-use for domestic livestock.

PLANT COMPOSITION CANOPY COVER(\%) MEAN RANGE CONST.

\begin{tabular}{llll}
$\begin{array}{l}\text { SHRUBS } \\
\text { WILLOW SPP }\end{array}$ & 37 & $0-85$ & 100 \\
$\begin{array}{l}\text { (Salix spp) } \\
\text { BOG BIRCH }\end{array}$ & 17 & $5-50$ & 100 \\
$\begin{array}{l}\text { (Betula glandulosa) } \\
\text { FORBS }\end{array}$ & 17 & & \\
$\begin{array}{l}\text { VEINY MEADOW RUE } \\
\text { (Thalictrum venulosum) }\end{array}$ & 2 & $0-9$ & 73 \\
$\begin{array}{l}\text { STRAWBERRY } \\
\text { (Fragaria virginiana) }\end{array}$ & 5 & $0-14$ & 73 \\
$\begin{array}{l}\text { FIREWEED } \\
\text { (Epilobium angustifolium)2 }\end{array}$ & $0-7$ & 73 \\
$\begin{array}{l}\text { LNNDLEY'S ASTER } \\
\text { (Aster ciliolatus) }\end{array}$ & 4 & $0-16$ & 55 \\
$\begin{array}{l}\text { GRASSES } \\
\text { BALTIC RUSH } \\
\text { (Juncus balticus) }\end{array}$ & 2 & $0-10$ & 90 \\
$\begin{array}{l}\text { GRACEFUL SEDGE } \\
\text { (Carex praegracilis) }\end{array}$ & 2 & $0-5$ & 54 \\
$\begin{array}{l}\text { SLENDER WHEATGRASS } \\
\text { (Agropyron trachycaulum)2 } \\
\text { TUFTED HAIRGRASS } \\
\text { (Deschampsia cespitosa) }\end{array}$ & 2 & $0-5$ & 55 \\
\hline
\end{tabular}

\section{ENVIRONMENTAL VARIABLES}

MOISTURE REGIME: SUBHYGRIC

NUTRIENT REGIME: MESOTROPHIC

ELEVATION: 1375-1646 M (1472 M)

SOIL DRAINAGE: IMPERFECTLY

ASPECT:

VARIABLE

SLOPE:

$0-5 \%$

\section{FORAGE PRODUCTION(KG/HA)}

GRASS: $1265(383-2966)$

FORBS: $811(200-1188)$

SHRUBS: $438(200-752)$

TOTAL: $2105(783-4292)$

\section{SUGGESTED GRAZING CAPACITY} NON-USE 


\section{UFB12. Willow-Alder/ Horsetail \\ (Salix bebbiana-Alnus tenuifolia/ Equisetum arvense)}

$\mathrm{n}=1$ This community type was described on the boundary between the Upper and Lower foothills subregions in the Solomon valley northwest of Hinton. It is very similar to the to the willow-alder/ shield fern-wild sarsaparilla community described by Lane et al (2000) in the Saddle Hills north of Grande Prairie. This community tends to occupy moist nutrient rich seepage areas which favour the growth of willow, horsetail and fern species. The high cover of willow and alder limits productivity of forbs and grass. It also limits access to domestic livestock. As a result this community type would be rated as non-use.

PLANT COMPOSITION CANOPY COVER(\%) MEAN RANGe Const.

\section{SHRUBS}

BEBB'S WILLOW

(Śalix bebbiana)

RIVER ALDER

(Alnus tenuifolia)

BRACTED HONEYSUCKLE

(Lonicera involcrata)

FORBS

HORSETAIL

(Equisetum arvense) 14

STRAWBERRY

(Fragaria virginiana) $\quad 1 \quad-\quad 100$

TALL LUNGWORT

(Mertensia paniculata)

LINDLEY'S ASTER

(Aster ciliolatus)

COW PARSNIP

(Heracleum lanatum)

GRASSES

TALL MANNA GRASS

(Glyceria grandis)

KENTUCKY BLUEGRASS

(Poa pratensis)
85

$15 \quad-\quad 100$

$5 \quad-\quad 100$

$14 \quad-\quad 100$

$1+100$

$3-100$

$1-100$

$2 \quad-100$

$1 \quad 100$

$1 \quad 100$

\section{ENVIRONMENTAL VARIABLES}

MOISTURE REGIME:

HYGRIC

NUTRIENT REGIME:

PERMESOTROPHIC

ELEVATION:

$1200 \mathrm{M}$

SOIL DRAINAGE :

IMPERFECTLY

FORAGE PRODUCTION(KG/HA)

GRASS: 162

FORBS: 1786

TOTAL: 1948

\section{SUGGESTED GRAZING CAPACITY NON-USE}


UPPER FOOTHILLS SUBREGION

\section{SHRUBLANDS AND GRASSLANDS MODIFIED BY GRAZING}

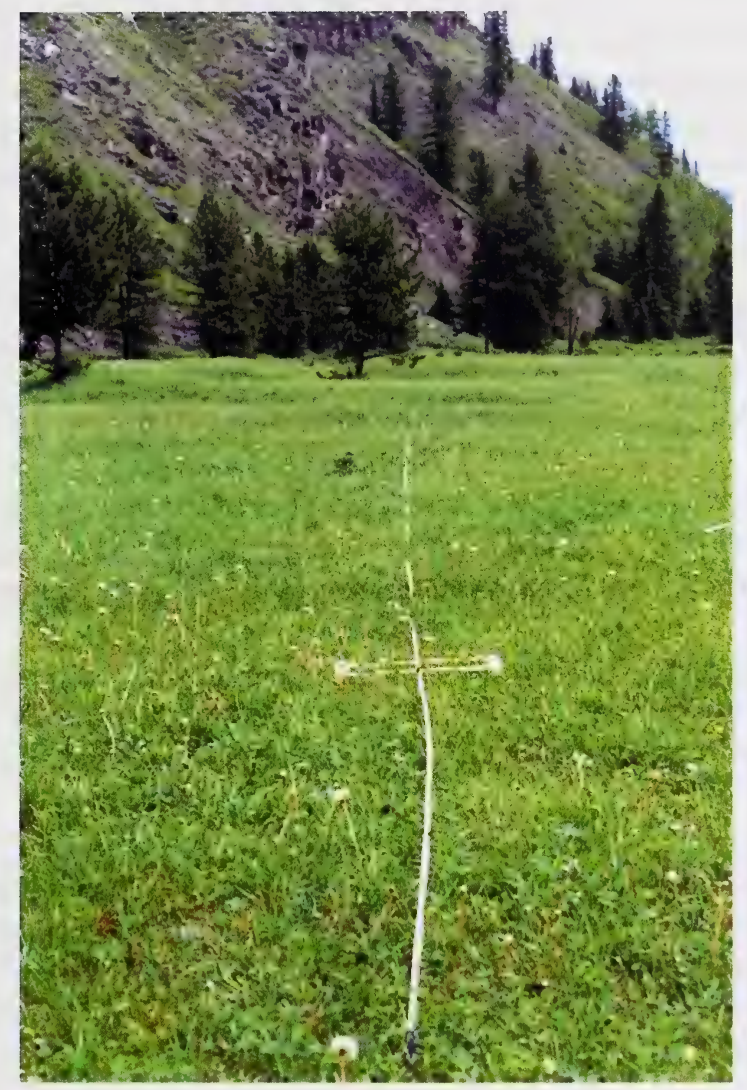

Figure 4. Heavily grazed Kentucky bluegrass/Dandelion community type 



\section{Grazing modified community types}

The grazing modified community types in the Upper Foothills subregion are outlined in Table 3. There are a few grasslands that exhibit signs of historic heavy grazing. These sites are predominantly covered by Kentucky bluegrass, timothy, dandelion and clover plant species (UFC3. Kentucky bluegrass-sedge/ dandelion and UFC4. Kentucky bluegrass/ dandelion and UFC8. Kentucky bluegrass-Timothy/Veiny meadow rue). Under long-term moderate grazing pressure or heavy grazing over a couple of years, there is a general decline in rough fescue and tufted hairgrass and an increase in sedge and slender wheatgrass (UFC1 Slender wheatgrass-sedge/ strawberry and UFC11.Sedge-Slender wheatgrass-Rough fescue). When these plant communities are protected from grazing, they appear to succeed back to the original communities dominated by rough fescue and tufted hairgrass. However, when Kentucky bluegrass becomes established, the community appears to revert to a rough fescue or tufted hairgrass-Kentucky bluegrass-dominated plant community (UFC5. Tufted hairgrass-Kentucky bluegrass) when protected from grazing.

The climax range condition model suggests that vegetation development will be directional, predictable and revert to the original vegetation when protected from grazing, but once Kentucky bluegrass has established, bluegrass appears to compete with rough fescue and tufted hairgrass for co-dominance. These Kentucky bluegrass communities move toward a different community rather than back to the original vegetation when protected from livestock disturbance.

The Rocky Mtn. fescue/ graceful cinquefoil community (UFC2) appears to be a moderately to heavily grazed California oatgrass community type. The dry, gravelly conditions on this site do not appear to favour the growth of Kentucky bluegrass under heavy grazing conditions.

The Creeping red fescue/ Clover (UFC7) community type represents seeded pastures and pipelines within the Upper Foothills subregion. This community type usually occurs at lower elevations, adjacent to farms and ranches where extensive modification of the native grass and shrublands have taken place.

The Purple oatgrass-Rough fescue (UFC9) community type was described in the Ghost area west of Calgary. It appears to represent a rough fescue, hairy wildrye dominated grassland that has undergone heavy grazing pressure. Willoughby (2000) has described purple oatgrass communities on saline soils in the Dry Mixedwood subregion. It is possible that this community type maybe associated with a saline seepage area which favours the growth of purple oatgrass. 


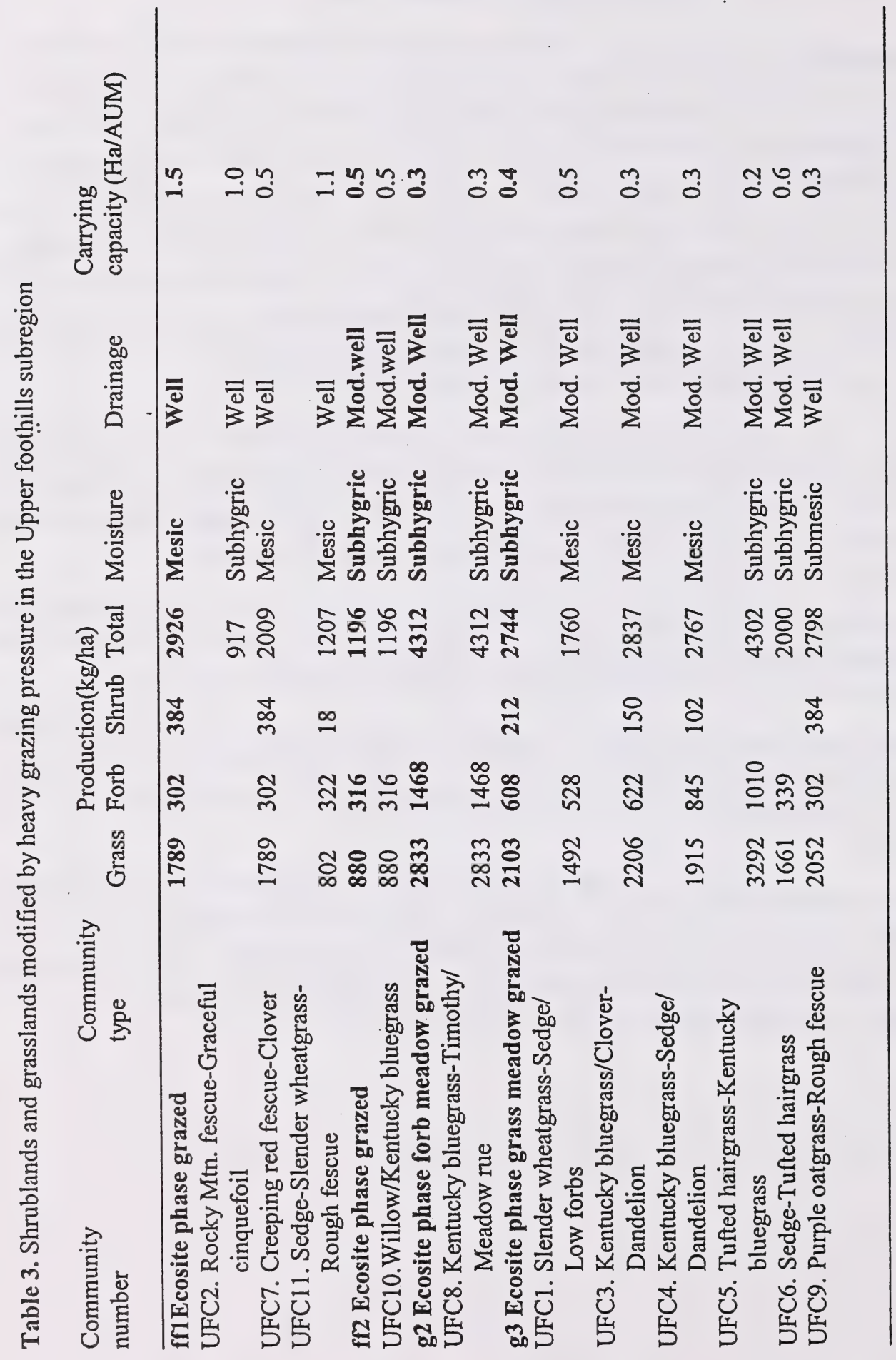

$\pi$ 


\section{UFC1. Slender wheatgrass-Sedge/ Low forbs \\ (Agropyron trachycaulum-Carex spp./ Fragaria virginiana)}

$\mathrm{n}=10$ This community type appears to arise from grazing a modal fescue-tufted hairgrass community (UFA5). Moderate to heavy grazing causes fescue and hairgrass, both decreasers, to decline in the stand. This community is very common in the valley bottoms in areas that are heavily utilized. While still quite productive, these sites have lost two of the most advantageous species. Only a reduction in grazing pressure will once again allow fescue and tufted hairgrass to become prevalent in the stand.

\section{PLANT COMPOSITION CANOPY COVER(\%)}

\section{MEAN RANGE CONST.}

\section{SHRUBS}

WILLOW

(Salix spp)

$4 \quad 0-25 \quad 30$

FORBS

STRAWBERRY

(Fragaria virginiana)

YARROW

(Achillea millefolium)

(Potentilla gracilis)

LINDLEY'S ASTER

(Aster ciliolatus)

DANDELION

(Taraxacum officinale)

MEADOW RUE

(Thalictrum venulosum)

GRASSES

SLENDER WHEATGRASS

(Agropyron trachycalum) $26 \quad 1-58 \quad 100$

TUFTED HAIRGRASS

(Deschampsia cespitosa) $1 \quad 0-6 \quad 10$

GRACEFUL SEDGE

(Carex praegracilis) $\quad 21 \quad 0-47 \quad 80$

KENTUCKY BLUEGRASS

(Poa pratensis)

HAIRY WILDRYE

(Elymus innovatus)

FRINGED BROME

(Bromus ciliatus)
$12 \quad 0-25 \quad 70$

$2-14 \quad 100$

$0-31 \quad 80$

$0-20 \quad 60$

$0-8 \quad 50$

$0-17 \quad 70$

$0-40-40$

$1 \quad 0 \div 4 \quad 40$

$5 \quad 0-15 \quad 50$

$9 \quad 0-56 \quad 40$

\section{ENVIRONMENTAL VARIABLES}

MOISTURE REGIME:

MESIC

NUTRIENT REGIME

MESOTROPHIC

ELEVATION:

SOIL DRAINAGE:

$1400-2438 \mathrm{M}(1623 \mathrm{M})$

MODERATELY WELL

ASPECT:

SOUTHERLY

SLOPE:

$0-27 \%$

\section{FORAGE PRODUCTION(KG/HA)}

GRASS: 1492 (824-2548)

FORBS: 528 (96-869)

TOTAL: $1760(920-2900)$

SUGGESTED GRAZING CAPACITY

0.5 HAAUM OR 1.2 ACRES/AUM 


\section{UFC2. Rocky Mountain fescue/ Graceful cinquefoil (Festuca brachyphylla/ Potentilla gracilis)}

$\mathrm{n}=1 \quad$ This community type was described on a gravelly, well drained site adjacent to Fall creek. It appears that this site was once a California oatgrass-sedge community type (UFA8), but heavy grazing pressure has shifted the community to one dominated by unpalatable low growing graminoids and forbs (Rocky mountain fescue, sedge, yarrow, graceful cinquefoil, pussy toes). The dry site conditions and poor nutrient conditions do not favour the growth of Kentucky bluegrass. This community type would benefit from a deferred rotational grazing system, where the community is rested every other year.

\section{Plant COMPOSITION CANOPY COVER(\%)}

\section{MEAN RANGe CONST.}

FORBS

WILD STRAWBERRY

(Fragaria virginiana)

GRACEFUL CINQUEFOIL

(Potentilla gracilis) $13 \quad-\quad 100$

YARROW

(Achillea millefolium) $8 \quad$ - $\quad 100$

ALPINE MILKVETCH

(Astragalus alpinus)

ROSEY PUSSY TOES

(Antennaria rosea)

RED SEEDED DANDELION

(Taraxacum laevigatum) 2

GRASSES

ROCKY MOUNTAIN FESCUE

(Festuca brachyphylla) 21

BROWNISH SEDGE

(Carex brunnescens) 5

SLENDER WHEATGRASS

(Agropyron trachycalum) $4 \quad$ - 100

CALIFORNIA OATGRASS

(Danthonia californica) $4 \quad 100$

\section{ENVIRONMENTAL VARIABLES}

MOISTURE REGIME:

SUBHYGRIC

NUTRIENT REGIME:

MESOTROPHIC

ELEVATION:

$1350 \mathrm{M}$

SOIL DRAINAGE :

WELL

FORAGE PRODUCTION(KG/HA)

TOTAL: 917

\section{SUGGESTED GRAZING CAPACITY}

1.0 HANAUM OR 2.2 ACRES/AUM 


\section{UFC3. Kentucky bluegrass/ Clover-Dandelion (Poa pratensis/Trifolium spp.-Taraxacum officinale)}

$\mathbf{n}=\mathbf{2 3}$ This community type develops when the modal tufted hairgrass-sedge dominated communities (UFA3, UFA4) are grazed heavily for prolonged periods of time. Willoughby (1992), felt these grasslands exhibited signs of historic heavy grazing pressure. He felt that under long-term moderate grazing or heavy grazing over a couple of years, rough fescue and tufted hairgrass decline and sedge, slender wheatgrass, and low growing forbs increase. When these plant communities are protected from grazing, they appear to succeed back to the original communities dominated by rough fescue and tufted hairgrass. However, when Kentucky bluegrass becomes established the community appears to revert to a rough fescue or tufted hairgrass-Kentucky bluegrass dominated plant community (UFC5).

These community types are highly productive for domestic livestock during the growing season, but the poor quality of Kentucky bluegrass, particularly in the dormant season, limits the use of these community types for wildlife.

\section{PLANT COMPOSITION CANOPY COVER(\%) MEAN RANGE CONST.}

\section{FORBS}

DANDELION

$\begin{array}{llll}\text { (Taraxacum officinale) } & 15 & 6-37 & 100\end{array}$

WILD STRAWBERRY

$\begin{array}{llll}\text { (Fragaria virginiana) } \quad 3 & 0-21 \quad 61\end{array}$

CLOVER

$\begin{array}{llll}\text { (Trifolium repens) } & 15 & 0-52 & 74\end{array}$

YARROW

(Achillea millefolium) $\quad 7 \quad 0-15 \quad 96$

GRACEFUL CINQUEFOIL

(Potentilla gracilis)

VEINY MEADOW RUE

(Thalictrum venulosum)

GRASSES

KENTUCKY BLUEGRASS

$\begin{array}{llll}\text { (Poa pratensis) } & 48 & 0-97 & 96\end{array}$

SLENDER WHEATGRASS

(Agropyron trachycaulum) $4 \quad 0-26 \quad 65$

CREEPING RED FESCUE

(Festuca rubra)

TUFTED HAIRGRASS

(Deschampsia cespitosa)

\section{ENVIRONMENTAL VARIABLES}

MOISTURE REGIME:

MESIC

NUTRIENT REGIME: MESOTROPHIC

ELEVATION:

$1150-1600 \mathrm{M}(1276 \mathrm{M})$

SOIL DRANAGE: MODERATELY WELL

ASPECT:

VARIABLE

SLOPE:

$0-35 \%$

FORAGE PRODUCTION(KG/HA)

GRASS: $2206(621-4319)$

FORBS: $622(153-2102)$

SHRUBS: 150

TOTAL: 2837 (1014-4686)

SUGGESTED GRAZING CAPACITY 0.3 HAAUM OR 0.7 ACRES/AUM 


\section{UFC4. Kentucky bluegrass-Sedge/ Dandelion (Poa pratensis-Carex spp./Taraxacum officinale)}

n=25 This community type is similar to the Kentucky bluegrass/ clover-dandelion community type (UFC3), but it has not been grazed as heavily. There is still an abundance of native plant species such as veiny meadow rue, slender wheatgrass, tufted hairgrass and sedge, but there has been an increase in grazing resistant species, such as Kentucky bluegrass, dandelion and clover. If this community type is protected from grazing it will probably revert back to a rufted haugrass-Kentucky bluegrass dominated type (UFC5) (Willoughby, 1992). Kentucky bluegrass, once established, appears to be a successful competitor.

These Kentusky bluegrass dominated community types are very productive, but they have lost two of the most advants ;eous species (tufted hairgrass and rough fescue). The forage quality of these native species is much better, particularly in the dormant season.

\section{PLANT ('MIPOSITION CANOPY COVER(\%) mean Range Const.}

\section{SHRLBS}

SHRUBBY CINOC I H :

(Potentilia fraticosa)

PRICKLYK JSE

(Rosa acicularis)

FORBS

DANDELON

(Taraxacum c. picinale) $\quad 24 \quad 1-53 \quad 100$

YARROW:

(Achillea millefolum) $9 \quad 1-25 \quad 100$

STRAWBERRY

(Fragario virginiana) $6 \quad 0-14 \quad 84$

VENY MEADOW' RUE

(Thalictrum venulosum) $\quad 11 \quad 0-41 \quad 80$

GRACEFUL CINQUEFOIL

$\begin{array}{llll}\text { (Potentilla gracilis) } & 14 & 0-40 & 88\end{array}$

GRASSES

KENTUCKY BLUEGRASS

(POa pratensis)

SLENDER WHEATGRASS

(Agropyron trachycaulum) $5 \quad 0-29 \quad 64$

TUFTED HAIRGRASS

(Deschampsia cespitosa) $6 \quad 0-21 \quad 72$

SEDGE SPP.

(Carex spp.)

$21 \quad 0-73 \quad 64$

CREEPING RED FESCUE

(Festuca rubra)

ROUGH FESCUE

(Festuca scabrella)
$1 \quad 0-5 \quad 12$

$4 \quad 0-12 \quad 60$

\section{ENVIRONMENTAL VARIABLES}

MOISTURE REGIME:

MESIC

NUTRIENT REGIME

PERMESOTROPHIC

ELEVATION:

$1150-1660 \mathrm{M}(1447 \mathrm{M})$

SOIL DRAINAGE:

MODERATELY WELL

FORAGE PRODUCTION(KG/HA)

GRASS: $1915(632-4304)$

FORBS: 845 (264-3344)

SHRUBS: 102

TOTAL: 2767 (1491-4864)

\section{SugGESTED GRAZING CAPACITY} 0.3 HAVAUM OR 0.7 ACRES/AUM 


\section{UFC5. Tufted hairgrass-Kentucky bluegrass \\ (Deschampsia cespitosa-Poa pratensis)}

$\mathrm{n}=4 \quad$ This community type is similar to the other Kentucky bluegrass dominated community types, but grazing pressure has been lighter or it was heavy and then became more moderate because of reduced stocking rates or rotational grazing. Willoughby (1992), found that tufted hairgrass could compete with Kentucky bluegrass in the absence of grazing, but it appears that once Kentucky bluegrass is established it remains to form a stable community type.

\section{PLANT COMPOSITION CANOPY COVER(\%)} MEAN RANGe CONST.

\section{SHRQUBS}

SHRUBBY CINQUEFOIL

$\begin{array}{llll}\text { (Potentilla fruticosa) } & 1 & 0-5 & 25\end{array}$

FORBS

DANDELION

(Taraxacum officinale) $\quad 13 \quad 3-21 \quad 100$

YARROW

$\begin{array}{llll}\text { (Achillea millefolium) } & 3 & \text { T-5 } & 100\end{array}$

STRAWBERRY

(Fragaria virginiana) $\quad 4 \quad 0-14 \quad 50$

VEINY MEADOW RUE

(Thalictrum venulosum) $2 \quad$ T-5 100

GRACEFUL CINQUEFOIL

(Potentilla gracilis) . $7 \quad 0-18 \quad 50$

GRASSES

KENTUCKY BLUEGRASS

$\begin{array}{llll}\text { (Poa pratensis) } & 12 & 2-19 & 100\end{array}$

SLENDER WHEATGRASS

(Agropyron trachycaulum) $5 \quad 0-10 \quad 75$

TUFTED HAIRGRASS

(Deschampsia cespitosa) $41 \quad 12-68 \quad 100$

SEDGE SPP.

(Carex spp.)

ROUGH FESCUE

(Festuca scabrella)

\section{ENVIRONMENTAL VARIABLES}

MOISTURE REGIME:

SUBHYGRC

NUTRIENT REGIME:

PERMESOTROPHIC

ELEVATION:

$1300-1600 \mathrm{M}(1400 \mathrm{M})$

SOIL DRAINAGE:

MODERATELY WELL

ASPECT:

NORTH

FORAGE PRODUCTION(KG/HA)

GRASS: 3292

FORBS: 1010

TOTAL: 4302

SUGGESTED GRAZING CAPACITY

0.2 HA/AUM OR 0.5 ACRES/AUM 


\section{UFC6. Sedge-Tufted hairgrass \\ (Carex spp.-Deschampsia cespitosa)}

n=13 This community type was described at Harrison Flats in the Upper Clearwater River valley. It appears to represent a tufted hairgrass-sedge community that was heavily grazed in the past and now is rested and only lightly utilized. It appears that the heavy grazing pressure was not prolonged enough to allow Kentucky bluegrass invasion. It is also possible that Kentucky bluegrass is not predominant on this site because of lack of seed source in these isolated areas. It is likely, with continued protection from grazing, that this community type will succeed back to a modal tufted hairgrass-sedge dominated community type.

\section{PLANT COMPOSITION CANOPY COVER(\%)} MEAN RANGe CONST.

SHRUBS

SHRUBBY CINQUEFOIL

(Potentilla fruticosa)

FORB

DANDELION

$\begin{array}{llll}\text { (Taraxacum officinale) } & 7 & 0-22 & 69\end{array}$

YARROW

(Achillea millefolium) $\quad 11 \quad 0-41 \quad 85$

STRAWBERRY

$\begin{array}{llll}\text { (Fragaria virginiana) } & 5 & 0-10 & 54\end{array}$

VEINY MEADOW RUE

(Thalictrum venulosum) $\quad 10 \quad 0-38 \quad 62$

GRACEFUL CINQUEFOIL

(Potentilla gracilis) $\quad 5 \quad 0-26 \quad 46$

GRASSES

KENTUCKY BLUEGRASS

(Poa pratensis)

SLENDER WHEATGRASS

(Agropyron trachycaulum)2 $\quad 0-13 \quad 31$

TUFTED HAIRGRASS

(Deschampsia cespitosa) 20

SEDGE

(Carex spp.)

$63 \quad 0-93 \quad 100$

ROUGH FESCUE

(Festuca scabrella)

$\begin{array}{lll}7 & 0-19 \quad 62\end{array}$

BALTIC RUSH

(Juncus balticus)

$15 \quad 0-58$
ENVIRONMENTAL VARIABLES

MOISTURE REGIME:

SUBHYGRIC

NUTRIENT REGIME:

MESOTROPHIC

ELEVATION:

SOIL DRAINAGE:

1529-1829 M (1781 M)

IMPERFECTLY

ASPECT:

VARIABLE

SLOPE:

$0 \%$

\section{FORAGE PRODUCTION(KG/HA)}

GRASS: $1020(684-1510)$

FORBS: $409(150-891)$

SHRUBS: 322

TOTAL: $1468(932-1962)$

0.6 HAAUM OR 1.4 ACRES/AUM 


\section{UFC7. Creeping red fescue-Clover (Festuca rubra-Trifolium spp.)}

$\mathrm{n}=23$ This community type was described at lower elevations, adjacent to farms and ranches in the Upper Foothills subregion. This community represents native communities that have been disturbed and planted to creeping red fescue. These include pipelines, roadsides and cultivated pastures. Lane et al. (2000), felt this community type developed when a site which was seeded to creeping red fescue-timothy-clover and received low levels of use. Creeping red fescue spreads throughout the site by creeping rhizomes and chokes out the timothy by forming a dense matt of litter. This community type is normally considered to be in good or excellent condition.

PLANT COMPOSITION CANOPY COVER(\%) MEAN RANGe Const.

\begin{tabular}{|c|c|c|c|}
\hline $\begin{array}{l}\text { FORBS } \\
\text { DAN்'DELION }\end{array}$ & & & \\
\hline $\begin{array}{l}\text { (Taraxacum officinale) } \\
\text { YARROW }\end{array}$ & 7 & $0-21$ & 88 \\
\hline $\begin{array}{l}\text { (Achillea millefolium) } \\
\text { STRAWBERRY }\end{array}$ & 2 & $0-7$ & 1 \\
\hline $\begin{array}{l}\text { (Fragaria virginiana) } \\
\text { CLOVER SPP. }\end{array}$ & 2 & $0-12$ & 63 \\
\hline $\begin{array}{l}\text { (Trifolium spp) } \\
\text { GRACEFUL CNQUEFOIL }\end{array}$ & 22 & $0-49$ & 100 \\
\hline $\begin{array}{l}\text { (Potentilla gracilis) } \\
\text { GRASSES }\end{array}$ & 1 & $0-7$ & 29 \\
\hline $\begin{array}{l}\text { KENTUCKY BLUEGRASS } \\
\text { (Poa pratensis) }\end{array}$ & 18 & $0-58$ & 83 \\
\hline $\begin{array}{l}\text { CREEPING RED FESCUE } \\
\text { (Festuca rubra) }\end{array}$ & 37 & $6-87$ & 100 \\
\hline $\begin{array}{l}\text { TUFTED HAIRGRASS } \\
\text { (Deschampsia cespitosa) }\end{array}$ & 1 & $0-4$ & 25 \\
\hline $\begin{array}{l}\text { SEDGE SPP. } \\
\text { (Carex spp.) }\end{array}$ & 2 & $0-11$ & 58 \\
\hline $\begin{array}{l}\text { HAIRY WILD RYE } \\
\text { (Elymus innovatus) }\end{array}$ & 2 & $0-17$ & 29 \\
\hline $\begin{array}{l}\text { TIMOTHY } \\
\text { (Phleum pratense) }\end{array}$ & 5 & $0-31$ & 71 \\
\hline
\end{tabular}

\section{ENVIRONMENTAL VARIABLES}

\author{
MOISTURE REGIME \\ MESIC \\ NUTRIENT REGIME: \\ MESOTROPHIC \\ ELEVATION: \\ $1212-1880 \mathrm{M}(1464 \mathrm{M})$ \\ SOIL DRAINAGE: \\ WELL \\ ASPECT:
VARIABLE
SLOPE:
$0-40 \%$

\section{FORAGE PRODUCTION(KG/HA)}

GRASS: 1789 (332-4894)

FORBS: 302 (20-1114)

SHRUBS: 384

TOTAL: 2009 (404-5054)

\section{SUGGESTED GRAZING CAPACITY 0.5 HAAUM OR 1.0 ACRES/AUM}




\section{UFC8. Kentucky bluegrass-Timothy/Meadow rue \\ (Poa pratensis-Phleum pratense/ Thalictrum venulosum)}

$\mathrm{n}=4$ This community type represents the grazed and disturbed community of the cow parsnip-meadow rue/ fringed brome community (UFA14). The high productivity and open nature of this community make it extremely attractive to domestic livestock. Heavy to moderate grazing pressure causes cow parsnip, veiny meadow rue and fringed brome to decrease and allows Kentucky bluegrass, timothy and dandelion to increase.

Plant COMPOSITION CANOPY COVER(\%) mean Range Const.

SHRUBS

WILLOW

(Salix spp.)

FORBS

DANDELION

(Taraxacum officinale) $\quad 13 \quad 2-35 \quad 100$

COW PARSNIP

(Heracleum lanatum) $\quad 7 \quad 0-23 \quad 50$

STRAWBERRY

(Fragaria virginiana) $1 \quad 0-1 \quad 50$

CLOVER

(Trifolium repens,

T. hybridum)

VEINY MEADOW RUE

(Thalictrum venulosum)

GRASSES

KENTUCKY BLUEGRASS

(Poa pratensis)

SLENDER WHEATGRASS

(Agropyron trachycaulum) 7

SMOOTH BROME

(Bromus inermis)

TIMOTHY

(Phleum pratense)
$2 \quad 0-5 \quad 75$

$5 \quad 0-20 \quad 25$

$\begin{array}{lll}7 & 0-24 & 50\end{array}$

$22 \quad 0-33 \quad 75$

$0-16 \quad 75$

$2 \quad 0-7 \quad 25$

$17 \quad 11-25 \quad 100$

\section{ENVIRONMENTAL VARIABLES}

MOISTURE REGIME:

MESIC

NUTRIENT REGIME:

PERMESOTROPHIC

ELEVATION:

1060-15201M (1330 M)

SOIL DRAINAGE:

MODERATELY WELL

ASPECT:

VARIABLE

SLOPE:

$0-6 \%$

\section{FORAGE PRODUCTION(KG/HA)}

GRASS: 2834 (308-6322)

FORBS: 1469 (210-2830)

SHRUBS: 42

TOTAL: $4313(560-6942)$

\section{SUGGESTED GRAZING CAPACITY}

0.3 HNAUM OR 0.7 ACRES/AUM 


\section{UFC9. Purple oatgrass-Rough fescue \\ (Schizachne purpurascens -Festuca scabrella)}

$\mathrm{n}=1$ This community type was described in the Ghost area west of Calgary. It appears to represent a rough fescue, hairy wildrye dominated grassland that has undergone heavy grazing pressure. Willoughby (1995) has found that purple oatgrass will increase with increased grazing pressure on nutrient poor soils in the Lower Foothills subregion. Willoughby (2000) also described a purple oatgrass-california oatgrass dominated community type on saline soils in the Dry Mixedwood subregion. It is possible that this community type maybe associated with a saline seepage area which favours the growth of purple oatgrass.

This community type is fairly productive, but the majority of the production is coming from purple oatgrass which is only moderately palatable to livestock. This community type should probably be rated as secondary range.

Plant COMPOSITION CANopy COVER(\%)

$\bullet$ MEAN Range Const.

\section{SHRUBS}

SHRUBBY CINQUEFOIL

(Potentilla fruticosa)

FORBS

THREE FLOWERED AVENS

(Geum triflorum)

GRACEFUL CINQUEFOIL

(Potentilla gracilis) $\quad 3 \cdot 100$

YARROW

(Achillia millifolium)

SMOOTH ASTER

(Aster laevis)

GRASSES

PURPLE OAT GRASS

(Schizachne purpurascens)15

SLENDER WHEATGRASS

(Agropyron trachycaulum)10

ROUGH FESCUE

(Festuca scabrella)

PRESL SEDGE

(Carex preslii)

JUNEGRASS

(Koeleria macrantha)
100

100

100

100

100

100

100

100

100

\section{ENVIRONMENTAL VARIABLES}

MOISTURE REGIME:

SUBMESIC

NUTRIENT REGIME:

MESOTROPHIC

ELEVATION:

1460

SOIL DRAINAGE:

WELL

ASPECT:

SOUTHERLY

SLOPE:

$25 \%$

\section{FORAGE PRODUCTION(KG/HA)}

GRASS: 2052

FORBS: 362

SHRUBS: 384

TOTAL: 2798

\section{SUGGESTED GRAZING CAPACITY} $0.3 \mathrm{Ha} / \mathrm{AUM}$ or $0.7 \mathrm{AC} / \mathrm{AUM}$ 


\section{UFC10. Willow/ Kentucky bluegrass \\ (Salix spp./Poa pratensis)}

$\mathrm{n}=2$ This community type represents the grazed and disturbed community of the willow/ tufted hairgrass-sedge community type (UFB3). The high productivity and open nature of this community make it extremely attractive to domestic livestock. Heavy to moderate grazing pressure causes the native plant species to decrease and allows Kentucky bluegrass and dandelion to increase.

Plant Composition Canopy Cover(\%) MEAN RANGe CONST.

SHRUBS

WILLOW

(Salix spp.)

FORBS

DANDELION

(Taraxacum officinale) $11 \quad 1-21 \quad 100$

YARROW

$\begin{array}{llll}\text { (Achillea millefolium) } & 7 & 5-7 & 100\end{array}$

STRAWBERRY

(Fragaria virginiana) $2 \quad$ T-4 100

WHITE CLOVER

(Trifolium repens)

TALL LUNGWORD

(Mertensia paniculata)

GRASSES

KENTUCKY BLUEGRASS

(Poa pratensis)

$22 \quad 19-25 \quad 100$

SLENDER WHEATGRASS

(Agropyron trachycaulum) $9 \quad 5-12 \quad 100$

TUFTED HAIRGRASS

(Deschampsia cespitosa) $8 \quad 0-15 \quad 50$

SHEEP FESCUE

$\begin{array}{llll}\text { (Festuca saximontana) } & 5 & 0-10 & 50\end{array}$
ENVIRONMENTAL VARIABLES

MOISTURE REGIME:

MESIC

NUTRIENT REGIME:

PERMESOTROPHIC

ELEVATION:

SOIL DRAINAGE:

1370-1667 M (1499 M)

WELL

ASPECT:

VARIABLE

SLOPE:

$0-25 \%$

FORAGE PRODUCTION(KG/HA)

GRASS: $1466(880-2052)$

FORBS: 339 (316-332)

SHRUBS: 384

TOTAL: $1997(1196-2798)$

SUGGESTED GRAZING CAPACITY

0.5 HNAUM OR 1.1 ACRES/AUM 


\section{UFC11. Sedge-Slender wheatgrass-Rough fescue \\ (Carex spp.-Agropyron trachycaulum-Festuca scabrella)}

$\mathbf{n = 2 9}$ This community type represents the grazed transects at the McCue Creek, Yara Creek and Upper James River rangeland reference areas over 30 years from the 1960 's to the early 1980 's. The continued grazing pressure since the 1980's has allowed Kentucky bluegrass to invade onto these sites to form a Kentucky bluegrass-Sedge dominated community type (Willoughby 2000). In the 1960's when these sites were protected from grazing the plant community succeeded back to a rough fescue-hairy wildrye dominated community.

\section{Plant COMPOSITION CANOPY COVER(\%)}

\section{MEAN RANGe CONST.}

\section{SHRUBS}

SHRUBBY CINQUEFOIL

(Potentilla fruticosa.)

FORBS

DANDELION

(Taraxacum officinale)

YARROW

(Achillea millefolium)

STRAWBERRY

$\begin{array}{llll}\text { (Fragaria virginiana) } & 2 & 0-18 & 45\end{array}$

GRACEFUL CINQUEFOIL

(Potentilla gracilis)

AMERICAN VETCH

(Vicia americana)

GRASSES

KENTUCKY BLUEGRASS

(Poa pratensis)

SLENDER WHEATGRASS

(Agropyron trachycaulum) 9

ROUGH FESCUE

(Festuca scabrella)

SEDGE SPP.

(Carex spp.)

HAIRY WILDRYE

(Elymus innovatus)
2

3

$0-13 \quad 90$

3

$0-7 \quad 97$

$\begin{array}{lll}3 & 0-8 & 79\end{array}$

$3 \quad 0-16 \quad 93$

$3 \quad 0-16 \quad 52$

$1-19 \quad 100$

$1-32 \quad 100$

$10 \quad 0-31 \quad 69$

$3 \quad 0-20 \quad 41$

\section{ENVIRONMENTAL VARIABLES}

MOISTURE REGIME: MESIC

NUTRIENT REGIME:

MESOTROPHIC

ELEVATION:

1470-1660(1543M)

SOIL DRAINAGE:

WELL

ASPECT:

SOUTHERLY

SLOPE:

$2-22 \%$

\section{FORAGE PRODUCTION(KG/HA)}

GRASS: 802

FORBS: 322

SHRUBS: 18

TOTAL: 1207

SUGGESTED GRAZING CAPACITY

1.1 HAAAUM OR 2.5 ACRES/AUM 



\section{UPPER FOOTHILLS SUBREGION}

\section{DECIDUOUS COMMUNITY TYPES}

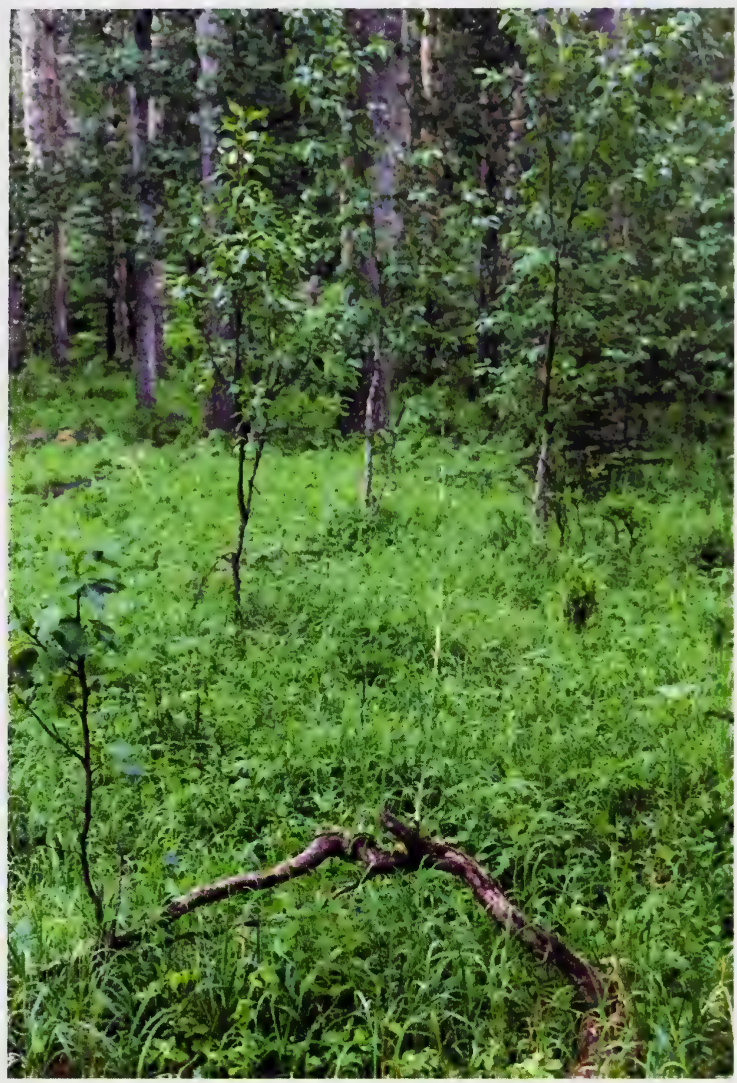

Figure 5. Aspen/Marsh reedgrass community type on south facing slopes in the Upper Foothills subregion 



\section{Deciduous community types}

The six deciduous community types described in the Upper Foothills subregion are outlined in Table 4. Deciduous types are rare in this subregion. The cool climate severely restricts the growth of deciduous tree species (Strong and Leggat 1992). As a result, aspen and balsam poplar are generally found on south facing slopes where the increased insolation permits colonization.

The $\mathrm{Pb}-\mathrm{Sw} / \mathrm{Willow} /$ Yellow Mtn. avens community type (UFD2) is representative of the gravelly floodplains adjacent to rivers and streams. The Aw/ Rose/ Bearberry, Aw/ Rose/ Hairy wildrye and Aw/ Buffaloberry/ Hairy wildrye community types are found on dry south facing slopes throughout the region. The Aw/ Buffaloberry/ Hairy wildrye community type appears to be successionally more advanced, with slightly acidic soils, than the Aw/ Rose/ Hairy wildrye community type. The Aw/ Marsh reedgrass type is slightly moister than the other aspen community types found on south facing slopes in the Upper Foothills subregion and the $\mathrm{Pb} /$ Willow/ Horsetail was described on the river floodplain adjacent to the Wildhay river. 


\section{Key to Deciduous Community Types}

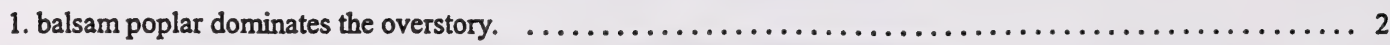

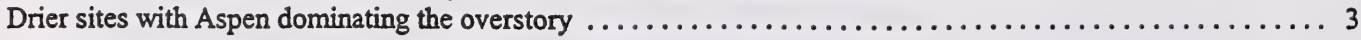

2. Low nutrient, dry gravelly river floodplains .. UFD2 Balsam poplar-White spruce/ Willow/ Yellow mtn. Avens Moist, moderately well drained soils ................. UFD6 Balsam poplar/ Willow/ horsetail

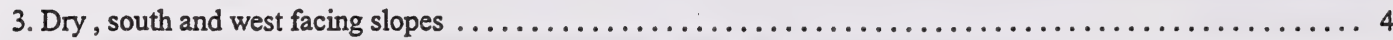
Moister richer sites with Marsh reedgrass dominated understory $\ldots \ldots \ldots \ldots$ UFD Aspen/Marsh reedgrass

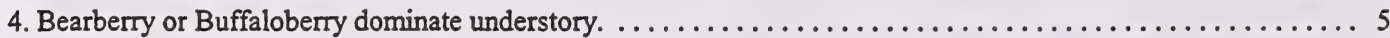
Grass and forbs dominate the understory of this dry, sunny site ........ UFD3 Aspen/ Rose/ Hairy wildrye

5. Dry site conditions on steep south facing slope $\ldots \ldots \ldots \ldots \ldots \ldots \ldots$ UFD1 Aspen/ Rose/ Bearberry Lower, south facing slopes and lower nutrient soils (high pH) ...... UFD4 Aspen/ Buffaloberry/ Hairy wildrye 


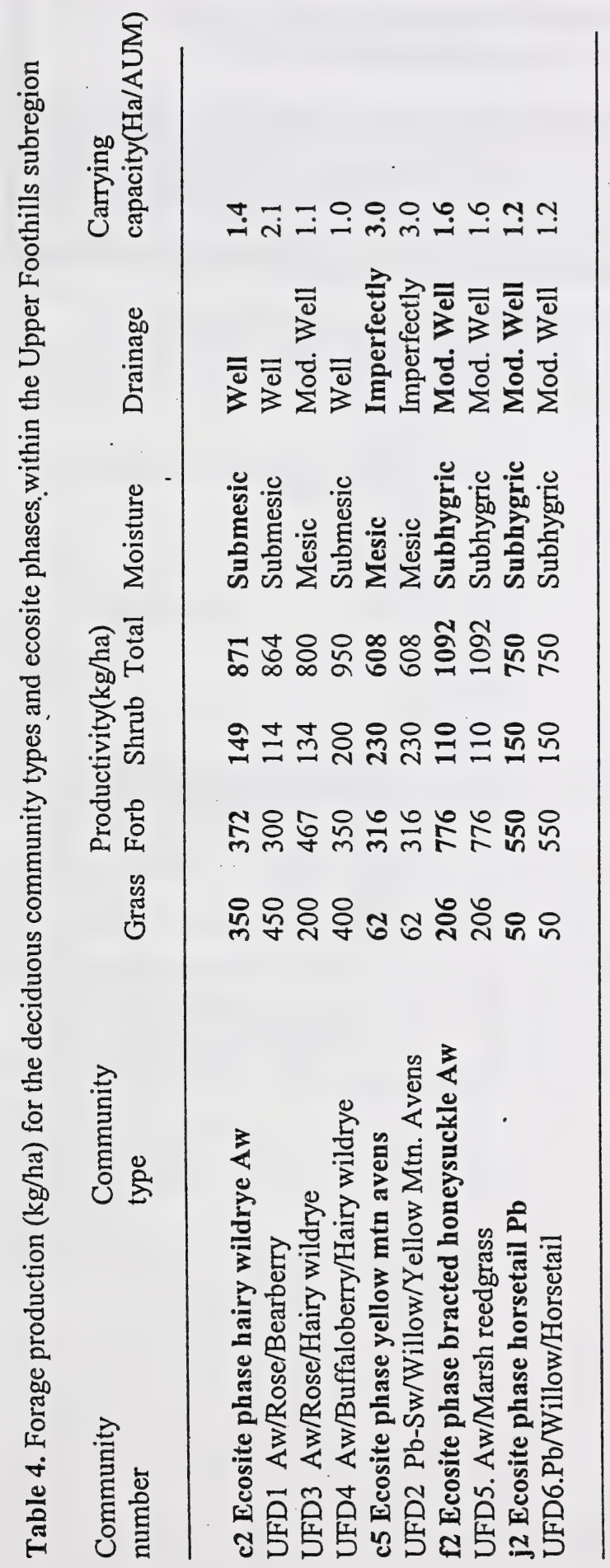




\section{UFD1. Aspen/ Rose/ Bearberry \\ (Populus tremuloides/Rosa acicularis/Arctostaphylos uva-ursi)}

n=1 This community type was described on the steep south facing slope above Rough Creek, west of Rocky Mountain House. The drier site conditions favour the growth of bearberry. This community type probably represents an earlier successional phase of the PV bearberry community type described by Beckingham et al (1996). The forage producuvity of this community type is only moderate, but the openness of the stand makes it accessible for livestock. This community type would be rated as secondary range.

\section{PLANT COMPOSITION CANopy COVER(\%) MEAN RANGe Const.}

TREES

ASPEN

(Populus tremuloides) 47

SHRL'BS

$47 \quad-\quad 100$

PRICKLY ROSE

(Rosa acicularas)

$22 \quad-\quad 100$

BEARBERRY

(Arctostophnlos u.a-ursi) 16

FORBS

STRAWBERRY

(Fragariaurginiana)

LINDLEY'S ASTER

(Aster cillolatus)

FIREWEED

(Epilobium angusfifolium)11 - 100

YARROW:

(Achilleo millefolium) $\quad 3 \quad-\quad 100$

GRASSES

SLENDER WHEATCRASS

(Agropyron trachycaulum)5 $\quad$ - 100

FRINGED BROME

(Bromus ciliotus) $\quad 3 \quad-\quad 100$

ROUGH FESCUE

(Festuca scabrella)

$3 \quad-100$

\section{ENVIRONMENTAL VARIABLES}

MOISTURE REGIME: MESIC

NUTRIENT REGIME: MESOTROPHIC

ELEVATION:

$1215 \mathrm{M}$

SOIL DRANAGE:

WELL

ASPECT:

SLOPE:

SOUTH-EAST

$30 \%$

\section{FORAGE PRODUCTION(KG/HA)}

GRASS: 450

FORBS: 300

SHRUBS: 114

TOTAL: 864

SUGGESTED GRAZING CAPACITY 2.1 HA/AUM OR 5.1 ACRES/AUM 


\section{UFD2. Balsam poplar/ Willow/ Yellow Mountain Avens (Populus balsamifera/ Salix spp./ Dryas drummondii)}

$\mathrm{n}=1$ This community type is common throughout the Upper Foothills subregion on gravelly floodplains along rivers and streams. It is similar to the bearberry/ slender wheatgrass community (UFA10), but it is successionally more advanced. This type is dominated by balsam poplar with an understory of spruce in the later successional stages. This particular stand was fairly young with the tree canopy being less than $5 \mathrm{~m}$ tall. Yellow mountain avens is a common pioneer species on gravelly river bars and rocky slopes up into the alpine tundra (MacKinnon et al., 1992). As this community succeeds towards a mature forest, yellow mountain avens will undoubtably decline in cover.

The forage production on this community type is very low. The poor nutrient status of the soil limits the growth of grasses, forbs and shrubs. As a result, this community type would be rated as non-use range.

\section{Plant COMPOSITION CANopy COVER(\%) \\ - MEAN RANGe CONST.}

\section{TREES}

BALSAM POPLAR

(Populus balsamifera) $7 \quad-\quad 100$

WHITE SPRUCE

(Picea glauca)

SHRUBS

WILLOW

(Salix spp.)

BUFFALOBERRY

(Shepherdia canadensis)

YELLOW MOUNTAIN AVENS

(Dryas drummondii) 16

BEARBERRY

(Arctostaphylos uva-ursi) 3

FORBS

WILD STRAWBERRY

(Fragaria virginiana)

ALPINE HEDYSARUM

(Hedysarum alpinum)

SCOURING RUSH

(Equisetum scirpoides) $11 \quad-\quad 100$

ALPINE MILKVETCH

(Astragalus alpinus) $\quad 4 \quad$ - 100

GRASSES

BLUNT SEDGE

(Carex obtusata)

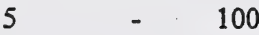

$13-100$

100

$-100$

$-100$

100

100

$2 \quad-\quad 100$

\section{ENVIRONMENTAL VARIABLES}

\section{MOISTURE REGIME:} MESIC

NUTRIENT REGIME: PERMESOTROPHIC

ELEVATION:

$1524 \mathrm{M}$

SOIL DRAINAGE:

IMPERFECTLY

\section{FORAGE PRODUCTION(KG/HA)}

GRASS: 62

FORBS: 316

SHRUBS: 230

TOTAL: 608

\section{SUGGESTED GRAZING CAPACITY 3 HNAUM OR 7 ACRES/AUM}




\section{UFD3. Aspen/ Rose/ Hairy wildrye \\ (Populus tremuloides/Rosa acicularis/Elymus innovatus)}

$\mathrm{n}=10$ This community type is typical of aspen forest types found throughout the Upper Foothills subregion on south facing slopes. The dry site conditions and high solar insolation favours the growth of grasses and forbs rather than shrubs. The canopy cover of aspen is also noticeably lower on this community type. This community type is similar to the Aw/ buffaloberry/ hairy wildrye community (UFD4)described in Willmore Wilderness Park, but the absence of buffaloberry distinguishes this type from the Willmore type. This community is moderately productive for domestic livestock. This community would be rated as secondary range.

Plant Composition Canopy Cover(\%) MEAN RANGE CONST.

TREES

ASPEN

(Populus tremuloides) $\quad 41 \quad 10-72 \quad 100$

SHRUBS

PRICKLY ROSE

(Rosa acicularis)

WILLOW.

(Salix spp.)

FORBS

LINDLEY'S ASTER

(Aster ciliolatus)

WILD STRAWBERRY

$\begin{array}{llll}\text { (Fragaria virginiana) } & 13 & 1-33 \quad 100\end{array}$

TALL LUNGWORT

(Mertensia paniculata) 4

CREAM COLORED VETCHLING

(Lathyrus ochroleucus) 3

VENY MEADOW RUE

(Thalictrum venulosum) 3

GRASSES

HAIRY WILD RYE

(Elymus innovatus)

$20 \quad 0-62 \quad 90$

PURPLE OATGRASS

(Schizachne purpurascens) $4 \quad 0-20 \quad 40$

SLENDER WHEATGRASS

(Agropyron trachycaulum) 4
0-12 100

$1-7 \quad 100$

$0-9 \quad 80$

$0-26 \quad 20$

\section{ENVIRONMENTAL VARIABLES}

MOISTURE REGIME

MESIC

NUTRIENT REGIME

MESOTROPHIC

ELEVATION:

$1345-1515 \mathrm{M}(1457 \mathrm{M})$

SOIL DRAINAGE (MEAN):

MODERATELY WELL

ASPECT:

SLOPE:

SOUTH TO SOUTHWEST

$0-35 \%$

FORAGE PRODUCTION(KG/HA)

GRASS: 200

FORBS: 467 (150-1000)

SHRUBS: $134(50-300)$

TOTAL: $800(401-1500)$

\section{SUGGESTED GRAZING CAPACITY}

1.1 HAAUM OR 2.5 AC/AUM 


\section{UFD4. Aspen/ Buffaloberry/ Hairy wildrye (Populus tremuloides/Shepherdia canadensis/ Elymus innovatus)}

$\mathrm{n}=3 \quad$ This community type was described along lower, south facing slopes and river terraces throughout Willmore Wilderness Park and areas west of Hinton. Bork (1994), found this community type. to be uncommon throughout Willmore, but pockets of this type were found along the Smoky, Sulphur and Sheep rivers on the North side of the Park. Bork felt that frequent disturbance and/ or arid conditions resulted in the aspen dominated overstory. He felt if left undisturbed, the community type would eventually succeed to a coniferous forest. This community type is very similar to the Aw/ buffaloberry type described by Youngblood (1993) in Alaska and the Aw/ rose/ hairy wildrye community type (UFD3) previously described near Rocky Mtn. House. The presence of buffaloberry distinguishes this northern type from the more southern rose type. The presence of buffaloberry may indicate a higher $\mathrm{pH}$ and lower nutrient status. Beckingham (1994), described Aw/ buffaloberry stands on lower $\mathrm{pH}$ sites.

This community type provides a good forage base for domestic livestock. In the Upper foothills, this community type is often located in close proximity to the trails and camps used by outfitters and recreationalists.

\section{Plant Composition Canopy Cover(\%) MEAN RaNGe Const.}

TREES

ASPEN

(Populus tremuloides) $\quad 34 \quad 24-52 \quad 100$

WHITE SPRUCE

(Picea glauca)

$4 \quad 0-11 \quad 33$

SHRUBS

BUFFALOBERRY

(Shepherdia canadensis) $\quad 14 \quad 10-18 \quad 100$

PRICKLY ROSE

(Rosa acicularis)

WILLOW

(Salix spp.)

TWIN-FLOWER

(Linnaea borealis)

BUNCHBERRY

(Cornus canadensis)

FORBS

FIREWEED

(Epilobium angustifolium) 3

SHOWY ASTER

(Aster conspicuus)

STRAWBERRY

(Fragaria virginiana)

GRASSES

HAIRY WILD RYE

(Elymus innovatus)

MARSH REEDGRASS

(C.alamagrostis canadensis)

$\begin{array}{lll}7 & 1-7 & 100\end{array}$

$17 \quad 5-36 \quad 100$

$1 \quad 0-4 \quad 33$

$2 \quad 0-7 \quad 33$

$1-5 \quad 100$

$1 \quad 0-4 \quad 33$

$9 \quad 3-19 \quad 100$

$24 \quad 14-34 \quad 100$
ENVIRONMENTAL VARIABLES

MOISTURE REGIME:

MESIC

NUTRIENT REGIME: MESOTROPHIC

ELEVATION:

914-1500 M (957 M)

SOIL DRAINAGE:

WELL

ASPECT:

SOUTH

SLOPE:

$0-10 \%$

FORAGE PRODUCTION(KG/HA)

GRASS: 400

FORBS: 350

SHRUBS: 200

TOTAL: 950

\section{SUGGESTED GRAZING CAPACITY 1 HNAUM OR 2.1 ACRES/AUM}




\section{UFD5. Aspen/ Marsh reedgrass \\ (Populus tremuloides/Calamagrostis canadensis)}

$\mathrm{n}=4 \quad$ This community type was described on a south facing slope in the Solomon valley west of Hinton and observed near Fall Creek and Upper James west of Rocky Mountain House. This community type is scattered throughout the valleys in small isolated areas. It appears to have a slightly higher moisture regime than the bearberry, hairy wildrye and buffaloberry dominated community types previously described. The dominance of marsh reedgrass indicates that some nutrient rich seepage occurs at some point in the growing season. This community type was located adjacent to Kentucky bluegrass-timothy dominated meadows (UFC8). As a result, this aspen dominated community type was extensively utilized by livestock.

PLANT COMPOSITION CANOPY COVER(\%) MEAN Range Const.

TREES

ASPEN

(Populus tremuloides)

BALSAM POPLAR

(Populus balsamifera)

WHITE SPRUCE

(Picea glauca)

SHRUBS

PRICKLY ROSE

(Rosa acicularis)

WILLOW

(Salix spp.)

FORBS

CANADA VIOLET

(Viola canadensis)

PEAVINE

(Lathyrus ochroleucus)

TALL LUNGWORT

(Mertensia paniculata)

LINDLEY'S ASTER

(Aster ciliolatus)

STRAWBERRY

$\begin{array}{llll}\text { (Fragaria virginiana) } \quad 4 & 1-9 & 100\end{array}$

COW PARNSIP

(Heracleum lanatum)

GRASSES

HAIRY WILD RYE

(Elymus innovatus)

MARSH REEDGRASS

(Calamagrostis canadensis)
29

14-45 100

$3 \quad 0-8 \quad 75$

$\begin{array}{lll}7 & 0-13 \quad 75\end{array}$

$1 \quad 0-3 \quad 75$

$\begin{array}{lll}3 & 0-8 & 50\end{array}$

$4 \quad 0-17 \quad 50$

$3 \quad 0-5 \quad 75$

3 T-6 100

$5 \quad 0-13 \quad 50$

$4 \quad 1-9 \quad 100$

$3 \quad 0-10 \quad 50$

$6 \quad 3-10 \quad 100$

$14 \quad 4-20 \quad 100$

\section{ENVIRONMENTAL VARIABLES}

MOISTURE REGIME:

SUBHYGRIC

NUTRIENT REGIME:

PERMESOTROPHIC

ElEVATION:

SOIL DRAINAGE:

$1450-1500 \mathrm{M}(1477 \mathrm{M})$

MODERATELY WELL

ASPECT:

WEST

SLOPE:

$3-18 \%$

\section{FORAGE PRODUCTION(KG/HA)}

GRASS: $206(110-301)$

FORBS: $776(350-1202)$

SHRUBS: $110(100-120)$

TOTAL: $1092(751-1432)$

\section{SUGGESTED GRAZING CAPACITY}

$1.6 \mathrm{HA} / \mathrm{AUM}$ OR $3.8 \mathrm{AC} / \mathrm{AUM}$ 


\section{UFD6. Balsam poplar/ Willow/ Horsetail \\ (Populus balsamifera/ Salix spp/ Equisetum arvense)}

$\mathrm{n}=1$ This community type was described on the flood plain of the Wildhay River northwest of Hinton. This community is not common in the Upper Foothills subregion and likely represents the continued succession of a willow/ horsetail dominated community type (UFB12). Continued succession in the absence of disturbance will likely lead to the development of a Sw/ horsetail dominated community type (UFE6).

This community type is being used by livestock because of its close proximity to a right of way that had been seeded to Creeping red fescue and clover. When in close proximity to primary range areas this community type should be rated as secondary range.

Plant CoMposition Canopy Cover(\%) MEAN RANGe Const.

TREES

ASPEN

(Populus tremuloides)

BALSAM POPLAR

(Populus balsamifera)

WHITE SPRUCE

(Picea glauca)

SHRUBS

PRICKLY ROSE

(Rosa acicularis)

BEAKED WILLOW

(Salix bebbiana)

FORBS

HORSETAIL

(Equisetum arvense)

SCOURNG RUSH

(Equisetum scirpoides)

TALL LUNGWORT

(Mertensia paniculata)

LINDLEY'S ASTER

(Aster cilioatus)

STRAWBERRY

(Fragaria virginiana)

RED CLOVER

(Trifolium pratense)

GRASSES

HAIRY WILD RYE

(Elymus innovatus)

MARSH REEDGRASS

(Calamagrostis canadensis)

KENTUCKY BLUEGRASS

(Poa pratensis)
4

7

4

1

100

100

$1 \quad-100$

\section{ENVIRONMENTAL VARIABLES}

MOISTURE REGIME:

SUBHYGRIC

NUTRIENT REGIME:

PERMESOTROPHIC

ELEVATION:

$1500 \mathrm{M}$

SOIL DRAINAGE:

MODERATELY WELL

\section{FORAGE PRODUCTION(KG/HA)}

GRASS: 50

FORBS: 550

SHRUBS: 150

TOTAL: 750

\section{SUGGESTED GRAZING CAPACITY}

$1.2 \mathrm{HA} / \mathrm{AUM}$ OR $2.7 \mathrm{AC} / \mathrm{AUM}$ 



\section{Conifer community types}

Lodgepole pine dominates the overstory vegetation of the mesic reference sites in the Upper Foothills subregion. Typical forests are represented by the PV/ bog cranberry (UFE1) and the PV/ marsh reedgrass c.t. (UFE4) community types. Secondary succession is by white spruce and leads to the formation of the Pl-Sw/ bunchberry c.t. (UFE2). Continued succession on wetter sites, in the absence of disturbance, leads to a Sw/ horsetail/moss dominated c.t. (UFE6) and to the Sw/moss (UFE10) dominated community on more mesic sites.

Wetter, subhygric sites can be dominated by lodgepole pine, black spruce or white spruce. Many of these sites have a predominant willow understory (PV/ willow/ moss (UFE3) or Sw/ willow(UFE7)). These types appear to represent continued succession from the native shrub and grassland community types. Succession in the absence of disturbance on these sites will be to white spruce. The Sw/ willow c.t. (UFE7) appears to be typical of a climax forest on these subhygric sites.

Bläck spruce dominates poorly drained depressional areas (Sb/ willow (UFE5)). These sites have a high water table throughout most of the year. Organic accumulations are a common result of the poor drainage conditions and low oxygen availability (Strong and Leggat, 1992).

Dry, south facing slopes are typically dominated by deciduous aspen forests with succession to a Sw/ bearberry (UFE8) and PV/ bearberry/ hairy wildrye dominated community types (Beckingham et al., 1996). A Sw/ juniper (UFE9) c.t. was described on fine-textured, calcareous loess deposits, with high pH's near Brule lake. These deposits blow out of the Athabasca river valley from Jasper National Park.

The conifer forest types are generally unsuitable for livestock grazing and are typically rated as nonuse. The ten coniferous community types described in the Upper Foothills subregion are outlined in Table 5. A more complete description of coniferous c.t. can be found in Beckingham et al. (1996). 


\section{Key to Coniferous Community Types}

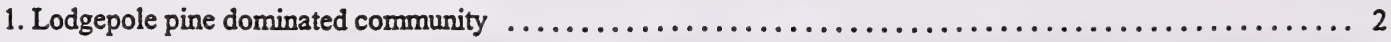

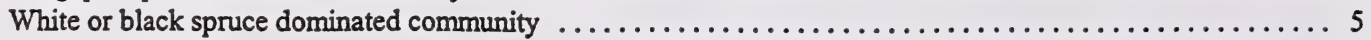

2. The community is supported by well drained, coarse soil. It is often located on south facing slopes and exhibits a

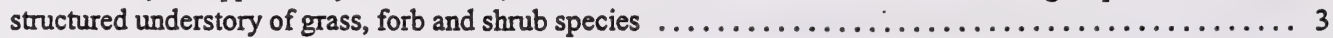
The community is located on less developed, wet soils which support a willow understory

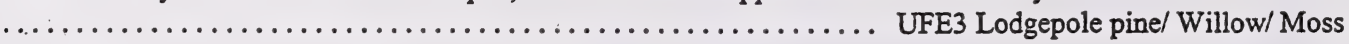

3. The community is succeeding to white spruce in undisturbed areas. Moss cover increases with canopy closure and grass, forb, and shrub species decline ............ UFE2 Lodgepole pine-White spruce/ Bunchberry

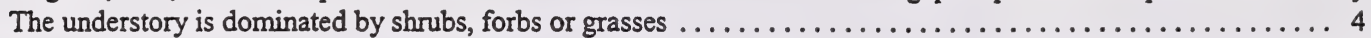

4. Shrubs dominate the understory on these well drained, south slopes ...... UFE1 Lodgepole pine/ Bog cranberry Forbs and grasses dominate the understory .............. UFE4 Lodgepole pine/ Marsh reedgrass

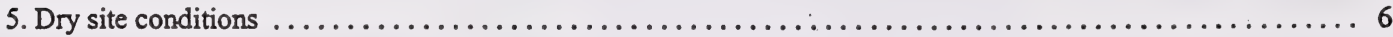

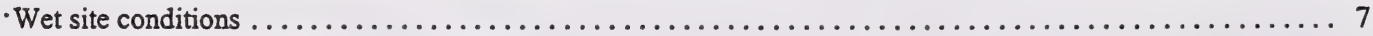

6. Poor nutrient regime, bearberry dominates the understory, wind ......... UFE8 White spruce/ Bearberry Fine textured calcareous loess with a high $\mathrm{pH} \ldots \ldots \ldots \ldots \ldots$ UFE9 White spruce/ Juniper-Buffaloberry

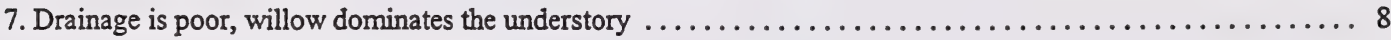

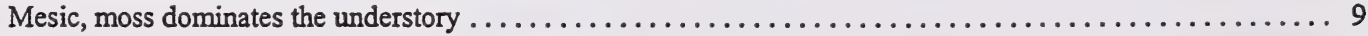

8. White spruce dominated overstory, wetter, low light Black spruce dominated overstory, wet, often saturated soils

9. Wet soils, canopy is closing, promoting moss and loss of forb etc. ..... UFE6 White spruce/ Horsetail/ Moss Closed canopy, successionally mature 


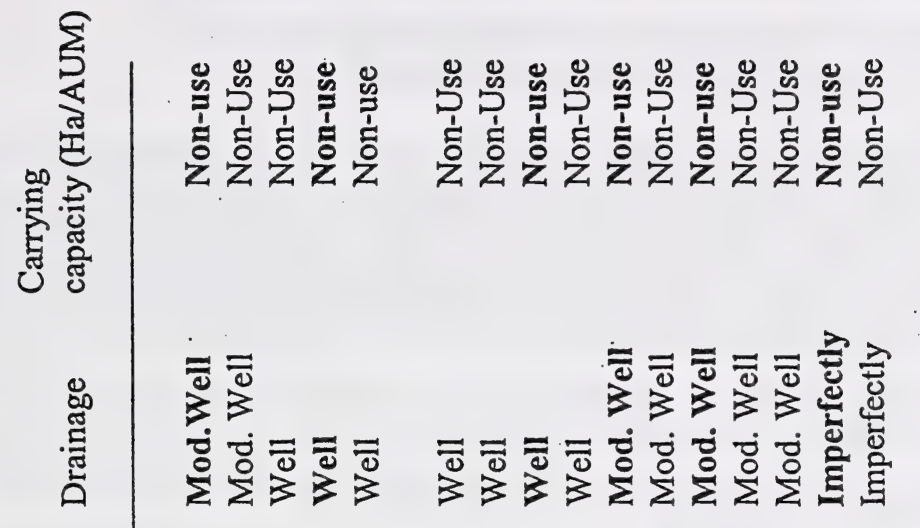

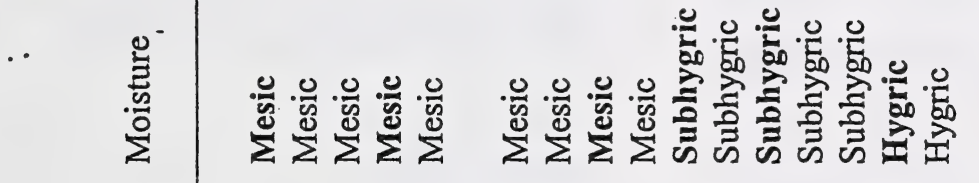

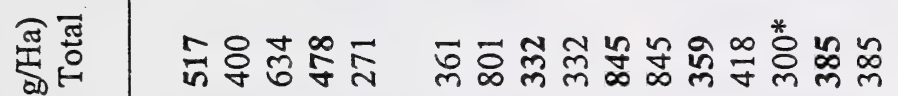
s.

.

으 응 की

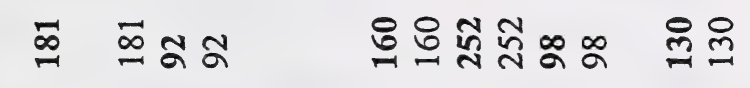

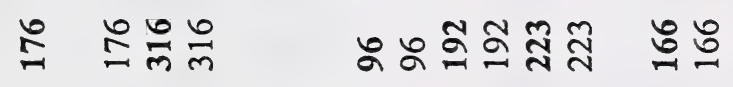

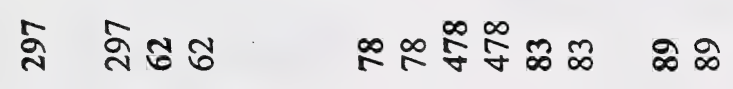

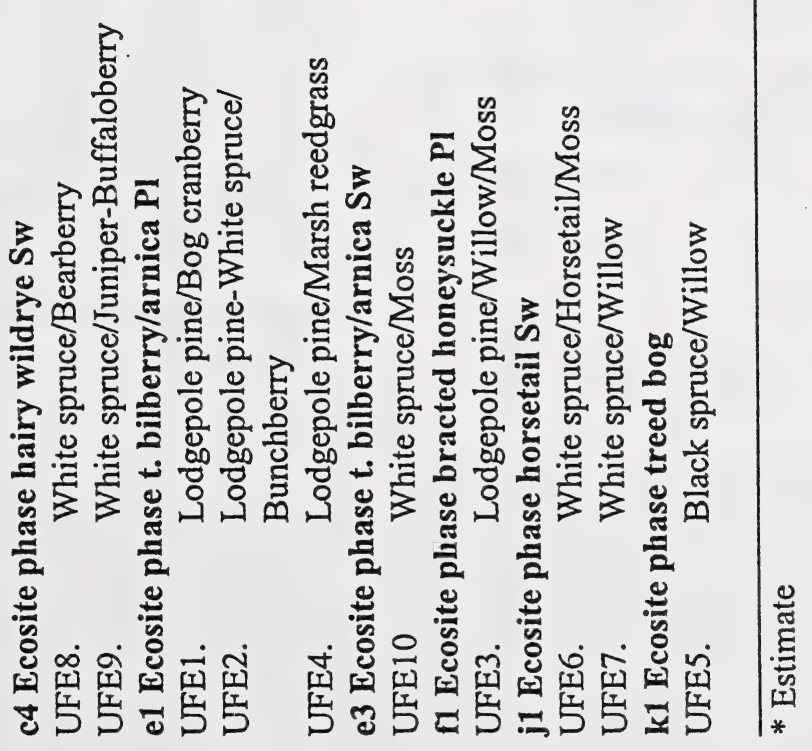




\section{UFE1. Lodgepole pine/ Bog cranberry \\ (Pinus contorta/ Vaccinium vitis-idaea)}

$\mathrm{n}=8 \quad$ This community type is common on dry, coarse textured, well drained sites throughout the Upper Foothills subregion and is part of the subxeric/ poor ecosite described by Beckingham et al. (1996). These sites are generally located on slopes with southerly aspects. This community type is very similar to the PV/ hairy wildrye/ bunchberry community type described by Lane et al. (2000) in the Lower Foothills subregion, and the Pl-Sw/ low bush cranberry/ twinflower type described by Beckingham (1994) in the Upper Foothills subregion, but this community type appears to be drier with a poorer nutrient regime. Beckingham(1994), felt that white spruce would eventually dominate the canopy of this community type.

Generally, this community type is not useful for domestic livestock grazing because it does not produce good quality forage.

\section{Plant CoMposition Canopy Cover(\%)} MEAN RANGe Const.

\section{TREES}

LODGEPOLE PINE

(Pinus contorta)

WHITE SPRUCE

(Picea glauca)

UNDERSTORY TREES

WHITE SPRUCE

(Picea glauca)

SHRUBS

BOG CRANBERRY

(Vaccinium vitis-idaea)

LABRADOR TEA

(Ledum groenlandicum)

DEWBERRY

(Rubus Pubescens)

BEARBERRY

(Arctostaphylos uva-ursi)

BUNCHBERRY

(Cornus canadensis)

TWINFLOWER

(Linnaea borealis)

GRASSES

HAIRY WILDRYE

(Elymus innovatus)

Moss

$\begin{array}{lll}35 & 20-50 & 100 \\ 7 & 0-20 & 50 \\ 2 & 0-15 & 25\end{array}$

$22 \quad 9-57 \quad 100$

$0-18 \quad 63$

$0-14 \quad 13$

$0-9 \quad 13$

$0-14 \quad 88$

$6 \quad 0-21 \quad 88$

6

63

\section{ENVIRONMENTAL VARIABLES}

\section{MOISTURE REGIME:}

MESIC

NUTRIENT REGIME:

ELEVATION: SUBMESOTROPHIC

SOIL DRAINAGE:

$$
\text { 1091-1475 M (1354 M) }
$$

WELL

ASPECT:

SLOPE:

VARIABLE

$0-15 \%$

\section{FORAGE PRODUCTION (KG/HA)}

GRASS: 62

FORBS: 316

SHRUBS: 92

TOTAL $271(89-470)$

\section{SUGGESTED GRAZING CAPACITY} NON-USE 


\section{UFE2. Lodgepole pine-White spruce/ Bunchberry (Pinus contorta-Picea glauca/ Cornus canadensis)}

$\mathrm{n}=5$ This community type represents the modal type on mesic/mesotrophic sites throughout the Upper Foothills subregion and may be transitional to the Lower Foothills subregion if aspen occurs in the stand. Strong (1992), found that lodgepole pine dominated the reference sites in this subregion with white spruce succession occurring on undisturbed areas. Beckingham (1994), described a similar community type (Pl-Sw/ low bush cranberry/ twinflower) and felt that white spruce and balsam fir will eventually dominate the canopy. The change in canopy dominance will lead to a decline in understory cover of shrubs and forbs. As succession occurs, moss cover will increase.

\section{This community type would be rated as non-use range for domestic livestock. There is little forage that}

\section{Plant CoMposition Canopy Cover(\%)}

\section{MEAN RANGe CONST.}

\section{TREES}

LODGEPOLE PINE

(Pinus contorta)

WHITE SPRUCE

(Picea glauca)

SHRUBS

DWARF BILBERRY

(Vaccinium caespitosum) 3

BOG CRANBERRY

(Vaccinium vitis-idaea)

WiLlow

(Salix spp.)

BUNCHBERRY

(Cornus canadensis) $\quad 21 \quad 2-39 \quad 100$

TWIN-FLOWER

(Linnaea borealis)

FORBS

WILD STRAWBERRY

(Fragaria virginiana) .

GRASSES

HAIRY WILD RYE

(Elymus innovatus)

MARSH REEDGRASS

(Calamagrostis

Moss

canadensis) $\quad 1 \quad 0-1 \quad 80$

\section{ENVIRONMENTAL VARIABLES}

MOISTURE REGIME:

MESIC

NUTRIENT REGIME:

MESOTROPHIC

ELEVATION:

$1091-1500 \mathrm{M}(1368 \mathrm{M})$

SOIL DRAINAGE:

WELL

ASPECT:

SOUTH

SLOPE:

$0-15 \%$

FORAGE PRODUCTION(KG/HA)

TOTAL: $361(288-496)$

\section{SUGGESTED GRAZING CAPACITY NON-USE}

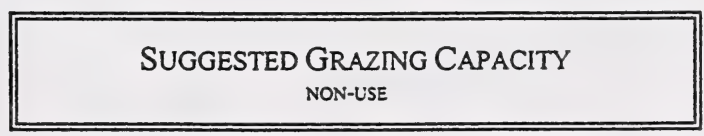




\section{UFE3. Lodgepole pine/ Willow/ Moss (Pinus contorta/Salix spp./Moss spp.)}

$\mathrm{n}=3 \quad$ This community type is very similar to the other lodgepole pine dominated community types, but it is found on wetter soils that lack development. This community type is slightly drier than the $\mathrm{Pl}-\mathrm{Sb} /$ labrador teawhortleberry/ bunchberry/ feather moss type described by Beckingham (1994) and the Sb/ willow dominated community type (UFE5) described in this guide. Herbaceous plants are scarce in the understory of this community type. As a result, there is little forage for domestic livestock and this community would be rated non-use.

\section{Plant COMPOSITION Canopy Cover(\%)} MEAN RANGE CONST.

\section{TREES}

LODGEPOLE PINE

(Pinus contorta)

WHITE SPRUCE

(Picea glauca)

SHRUBS

WILLOW

(Salix spp.)

BUNCHBERRY

$\begin{array}{llll}\text { (Cornus canadensis) } & 4 & 1-6 & 100\end{array}$

TWIN-FLOWER

(Linnaea borealis)

FORBS

WILD STRAWBERRY

Fragaria virginiana) $\quad 3 \quad 1-4 \quad 100$

PALMATE LEAVED COLTSFOOT

$\begin{array}{llll}\text { (Petasites palmatus) } & 1 & \text { T } & 100\end{array}$

GRASSES

HAIRY WILD RYE

(Elymus innovatus)

MARSH REEDGRASS

(Calamagrostis canadensis)2

Moss

$\begin{array}{lll}32 & 25-40 & 100 \\ 13 & 5-30 & 100\end{array}$

$23 \quad 13-34 \quad 100$

$1 \quad 0-3 \quad 33$

\section{ENVIRONMENTAL VARIABLES}

MOISTURE REGIME:

SUBHYGRIC

NUTRIENT REGIME:

PERMESOTROPHIC

ELEVATION:

SOIL DRAINAGE:

$1390-1560 \mathrm{M}(1451 \mathrm{M})$

MODERATELY WELL

ASPECT:

NORTHERLY

SLOPE:

$0-10 \%$

\section{FORAGE PRODUCTION(KG/HA)}

GRASS: $478(283-672)$

FORBS: $192(170-214)$

SHRUBS: 252 (204-300)

TOTAL: 845 (644-1046)

SUGGESTED GRAZING CAPACITY NON-USE 


\section{UFE4. Lodgepole pine/ Marsh reedgrass (Pinus contorta/ Calamagrostis canadensis)}

$\mathrm{n}=3$ This community type is similar to the $\mathrm{Pl} /$ hairy wildrye/ fireweed-peavine community type described by Lane et al. (2000). The tree canopy is open which allows good understory growth.

The good understory forage production and easy access through this community type makes it useful for livestock grazing. If this community type occurs adjacent to a physical feature that attracts livestock to the area, it may be considered primary range.

Plant COMPOSITION CANOPY Cover(\%) MEAN RANGE CONST.

\section{TREES}

LODGEPOLE PINE

(Pinus contorta)

WHITE SPRUCE

(Picea glauca)

SHRUBS

PRICKLY ROSE

(Rosa acicularis)

BOG CRANBERRY

(Vaccinium vitis-idaea)

BUNCHBERRY

(Cornus canadensis)

TWIN-FLOWER

(Linnaea borealis)

FORBS

FIREWEED

(Epilobium angustifolium)

LINDLEY'S ASTER

(Aster ciliolatus)

GRASSES

HAIRY WILD RYE

(Elymus innovatus)

MARSH REEDGRASS

(Calamagrostis

Moss

$13 \quad 0-30 \quad 67$

$12 \quad 0-20 \quad 67$

1 T-2 100

$2 \quad 0-7 \quad 33$

$5 \quad 2-9 \quad 100$

$4 \quad$ T-6 100

$2-3 \quad 100$

T-6 100

$3 \quad T-6 \quad 100$

$5 \quad 2-6 \quad 100$

$12 \quad 4-18 \quad 100$

$12 \quad 8-17 \quad 100$

\section{ENVIRONMENTAL VARIABLES}

MOISTURE REGIME:

MESIC

NUTRIENT REGIME:

MESOTROPHIC

ELEVATION:

1350-1380 M (1367) M

SOIL DRANAGE:

WELL

ASPECT:

VARIABLE

SLOPE:

$0-45 \%$

FORAGE PRODUCTION(KG/HA)

TOTAL: $801(600-1200)$

SugGeSTED GRAZING CAPACITY

NON-USE 


\section{UFE5. Black spruce/ Willow (Picea mariana/ Salix spp.)}

$\mathbf{n}=2$ This community type is characterized by a dominant cover of black spruce and a sparse understory cover. The sites are moist in the spring and dry out later in the growing season. Corns and Annas (1986), found that these forests have a fire origin and can persist for more than 150 years.

This community type would be considered non-use for domestic livestock.

Plant CoMposition Canopy Cover(\%) MEAN RANGE CONST.

\section{TREES}

BLACK SPRUCE

(Picea mariana)

WHiITE SPRUCE

(Picea glauca)

SHRUBS

WILLOW SPP.

(Salix spp.)

LABRADOR TEA

(Ledum groenlandicum)

BUNCHBERRY

(Cornus canadensis)

FORBS

PaLMATE LEAVEd COLTSFOOT

(Petasites palmatus) 1

WILD STRAWBERRY

(Fragaria virginiana) 1

HORSETAIL

(Equisetum spp)

GRASSES

WATER SEDGE

(Carex aquatilis)

GRACEFUL SEDGE

(Carex praegracilis)

Moss

$15 \quad 10-20 \quad 100$

$\begin{array}{lll}6 & 2-10 \quad 100\end{array}$

$49 \quad 32-65 \quad 100$

$0-14 \quad 50$

$0-13 \quad 50$

$0-1 \quad 50$

T-1 100

$\begin{array}{lll}8 & \mathrm{~T}-8 \quad 100\end{array}$

$5 \quad 0-10 \quad 50$

$4 \quad 0-8 \quad 50$

$\begin{array}{lll}51 & 41-59 & 100\end{array}$

\section{ENVIRONMENTAL VARIABLES}

MOISTURE REGIME:

HYGRIC

NUTRIENT REGIME:

PERMESOTROPHIC

ELEVATION:

1415-1454 M (1435) M

SOIL DRAINAGE:

IMPERFECTLY

ASPECT:

NORTH

SLOPE:

$0-10 \%$

FORAGE PRODUCTION(KG/HA)

GRASS: $89(62-116)$

FORBS: $166(15-316)$

SHRUBS: $130(30-230)$

TOTAL: 385 (161-608)

SUGGESTED GRAZING CAPACITY NON-USE 


\section{UFE6. White spruce/ Horsetail/ Moss \\ (Picea glauca/ Equisetum arvense/ Moss spp.)}

$\mathrm{n}=3$ This community type is successionally more advanced than the Pl-Sw/ bunchberry community type (UFE2) previously described. The lack of fire disturbance has allowed white spruce to succeed into the lodgepole pine canopy and dominate the site. As these stands mature, their canopies close, shading the understory vegetation and allowing moss cover to increase. The sparseness and low palatablity of the vegetation limits the use of these stands by domestic livestock.

PLANT COMPOSITION CANOPY COVER(\%) Mean Range Const.

\section{TREES}

WHITE SPRUCE

(Piçea glauca) ${ }^{-}$

$43 \quad 15-65 \quad 100$

BÄLSAM POPLAR

(Populus balsamifera)

UNDERSTORY TREES

BALSAM POPLAR

(Populus balsamifera)

SHRUBS

LOW BUSH CRANBERRY

(Viburnum edule)

ROSE

(Rosa acicularis)

TWINFLOWER

(Linnaea borealis)

FORBS

PALMATE LEAVED COLTSFOOT

(Petasites palmatus) 11

SCOURING RUSH

(Equisetum scirpoides) 9

HORSETAIL

$\begin{array}{llll}\text { (Equisetum arvense) } \quad 9 & 9-12 & 100\end{array}$

TALLLUNGWORT

(Mertensia paniculata)

GRASSES

HAIRY WILDRYE

(Elymus innovatus)

Moss

$2 \quad 0-3 \quad 67$

$0-5 \quad 33$

$0-3 \quad 67$

$0-14 \quad 67$

$0-9 \quad 67$

$0-22 \quad 67$

$0-16 . \quad 67$

$0-4 \quad 67$

$2 \quad 0-4 \quad 67$

$\begin{array}{lll}5 & 3-7 & 100\end{array}$

$37 \quad 0-91 \quad 67$

\section{ENVIRONMENTAL VARIABLES}

MOISTURE REGIME:

SUBHYGRIC

NUTRIENT REGIME:

MESOTROPHIC

ELEVATION:

SOIL DRAINAGE:

1350-1454 M (1415 M)

MODERATELY WELL

ASPECT:

NORTHEAST ·

SLOPE:

$3 \%$

\section{FORAGE PRODUCTION(KG/HA)}

GRASS: $83(68-96)$

FORBS: $223(212-234)$

SHRUBS: 98 (0-196)

TOTAL: $418(332-504)$

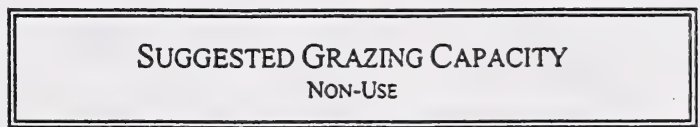




\section{UFE7. White spruce/ Willow \\ (Picea glauca/ Salix spp.)}

$\mathrm{n}=1$ This community type is similar to the Sw/ bunchberry/ moss community type, but is found on wetter sites, with poorer drainage. The wetter sites favour the growth of willow in the understory. The high cover of willow and spruce limits the amount of light reaching the understory. Consequently, there is little forage for domestic livestock.

PLANT COMPOSITION CANOPY COVER(\%) MEAN RANGe CONST.

TREES

WHITE SPRUCE

(Picea glauca)

LODGEPOLE PINE

(Pinus contorta)

SHRUBS

WILLOW

(Salix spp.)

BOG BIRCH

(Betula glandulosa)

LOW BILBERRY

(Vaccinium caespitosum) 6

TWIN-FLOWER

(Linnaea borealis) 5

FORBS

CREAM COLORED VETCHLING

(Lathyrus ochroleucus) 2

LINDLEY'S ASTER

(Aster ciliolatus)

WILD STRAWBERRY

(Fragaria virginiana)

ALPINE ASTER

(Aster alpinus)

YARROW

(Achillea millefolium) $3 \quad-\quad 100$

FIREWEED

(Epilobium angustifolium) $3 \quad-\quad 100$

GRASSES

GRACEFUL SEDGE

(Carex praegracilis) $\quad 7 \quad-\quad 100$

HAIRY WILD RYE

(Elymus innovatus)
$45 \quad-\quad 100$

$10 \quad-\quad 100$

$60 \quad-\quad 100$

$8-100$

$-\quad 100$

100

100

100

100

100

100

\section{ENVIRONMENTAL VARIABLES}

MOISTURE REGIME:

SUBHYGRIC

NUTRIENT REGIME:

PERMESOTROPHIC

ELEVATION:

$1646 \mathrm{M}$

SOIL DRANAGE:

MODERATELY WELL

ASPECT:

WEST

SLOPE:

$15 \%$

FORAGE PRODUCTION(KG/HA)

TOTAL: 300

SUGGESTED GRAZING CAPACITY

NON-USE 


\section{UFE8. White spruce/ Bearberry \\ (Picea glaucal Arctostaphylos uva-ursi)}

$\mathrm{n}=1$ This community type is similar to the $\mathrm{Sw} /$ buffaloberry/ bearberry c.t. described by Lane et al. (2000) in the Lower Foothills. This type is fairly dry with a poor nutrient regime; as indicated by the high abundance of bearberry. It may also be somewhat windswept and desiccated, as indicated by the low tree canopy cover.

If this community type is located near a physical feature that attracts livestock to the area it may be considered to be pramary or secondary range. In other instances though, where it is not near an attractive feature, this communty npe would be considered non-use.

PlaNt COMPOSITION CANOPY COVER(\%) MEAN RANGe CONST.

\section{TREES}

WHITE SPR:CI

(Piceo gluaca)

ASPEN

(Populus tremulobies) $8 \quad-\quad 100$

SHRLBS

SHRUBBY CN DUFFOI:

(Potentilla fruscosa)

WILLO SPP

(Salix SPP)

BOC BIRCH

(Betulagiondulosa)

BEARBERRY

(Arctostaphylos wva-ursi) $23 \quad-\quad 100$

FORBS

SHOWY LOCOWEED

(Oxytropis splendens)

WILD STRAWBERRY

(Fragaria virginiana)

ALPINE MILKVETCH

(Astragalus alpinus)

CLOVER

(Trifolium repens) 6

DANDELION

(Taraxacum officinale)

GRASSES

PURPLE OATGRASS

(Schizachne purpurascens)18 - 100

SLENDER WHEATGRASS

(Agropyron trachycaulum) $14 \quad$ - 100

BLUNT SEDGE

(Carex obtustata)

\section{ENVIRONMENTAL VARIABLES}

MOISTURE REGIME:

MESIC

NUTRIENT REGIME:

MESOTROPHIC

ELEVATION:

$1311 \mathrm{M}$

SOIL DRAINAGE:

MODERATELY WELL

FORAGE PRODUCTION(KG/HA)

TOTAL: 400

SugGESTED GRAZING CAPACITY

NON-USE 


\section{UFE9. White spruce/ Juniper-Buffaloberry \\ (Picea glauca/ Juniperus horizontalis-Shepherdia canadensis)}

$\mathbf{n}=\mathbf{2}$ This community type was described along the north shore of Brule lake. It is characteristic of the finetextured, calcareous loess deposits which have blown down the Athabasca river valley from Jasper National Park. The soils of this community have a high $\mathrm{pH}(8)$ which supports a good cover of hairy wildrye. This community type is extremely slow growing. When harvested, the cutblocks resemble native grasslands (juniper/ hairy wildrye (UFF1) and rose/ hairy wildrye (UFF2)).

\section{Plant Composition CANopy COVER(\%)}

\section{MEAN RANGe CONST.}

\section{TREES}

WHITE SPRUCE

(Picea glauca) -

SHRंUBS

SHRUBBY CINQUEFOIL

(Potentilla fruticosa)

CREEPING JUNIPER.

(Juniperus horizontalis)

PRICKLY ROSE

(Rosa acicularis)

BUFFALOBERRY

(Shepherdia canadensis)

FORBS

WHITE CAMAS

(Zigadenus elegans)

SHOWY LOCOWEED

(Oxytropis splendens)

NORTHERN HEDYSARUM

(Hedysarum boreale)

(Galium boreale)

BASTARD TOAD FLAX

(Comandra umbellata)

GRASSES

HAIRY WILDRYE

(Elymus innovatus)

BLUNT SEDGE

(Carex obtustata)

$50 \quad 50 \quad 100$

$3 \quad 1-4 \quad 100$

$44 \quad 43-45 \quad 100$

$7 \quad 6-8 \quad 100$

$3 \quad 0-5 \quad 50$

$4 \quad \mathrm{~T}-7 \quad 100$

$2 \quad \mathrm{~T}-3 \quad 100$

$2 \quad 0-4 \quad 50$

$2 \quad 1-2 \quad 100$

$1 \quad 1-2 \quad 100$

$14 \quad 13-14 \quad 100$

$4 \quad 3-5 \quad 100$

\section{ENVIRONMENTAL VARIABLES}

MOISTURE REGIME: MESIC

NUTRIENT REGIME: MESOTROPHIC

ELEVATION: $1066 \mathrm{M}$

SOIL DRAINAGE: WELL

ASPECT: SOUTH

SLOPE:

$$
0-5 \%
$$

\section{FORAGE PRODUCTION(KG/HA)}

GRASS: 297 (294-300)

FORBS: $176(146-206)$

SHRUBS: 181 (36-326)

TOTAL: 654 (536-772)

\section{SUGGESTED GRAZING CAPACITY NON-USE}




\section{UFE10. White spruce/ Moss \\ (Picea glauca/ Moss spp.)}

$\mathrm{n}=1$ This community type represents a successionally mature forested stand in the Upper Foothills subregion As succession occurs from pine to spruce, the canopy cover becomes closed and the amount of understory vegetation decreases until most of the shrub, forb and grass layers have been eliminated. As a result, there is limited forage available for domestic livestock within these spruce dominated community types. This community is typically rated as non-use for domestic livestock.

\section{Plant COMPOSITION CANOPY COVER(\%) MEAN RANGE CONST.}

\section{TREES}

WHITE SPRUCE

(Picea glauca) -

LODGGEPOLE PINE

(Pinus contorta)

UNDERSTORY TREES

SUBALPINE FIR

(Abies lasiocarpa)

SHRUBS

BUFFALOBERRY

(Shepherdia canadensis)

BOG CRANBERRY.

(Vaccinium caespitosum) 4

PRICKLY ROSE

(Rosa acicularis)

TWINFLOWER

(Linnaea borealis)

FORBS

HEART LEAVED ARNICA

(Arnica cordifolia)

SHOWY ASTER

(Aster conspicuus)

BUNCHBERRY

(Cornus canadensis)

STRAWBERRY

(Fragaria virginiana) $2 \quad-\quad 100$

GRASSES

HAIRY WILDRYE

(Elymus innovatus)

Moss

STAIR STEP MOSS

(Hylocomium splendens)

\section{ENVIRONMENTAL VARIABLES}

MOISTURE REGIME:

MESIC

NUTRIENT REGIME:

MESOTROPHIC

ELEVATION:

$1350 \mathrm{M}$

SOIL DRAINAGE:

WELL

ASPECT:

EAST

SLOPE:

$10 \%$

\section{FORAGE PRODUCTION(KG/HA)}

GRASS: 78

FORBS: 96

SHRUBS: 160

TOTAL: 332

NON-USE 


\section{Cutblock and burn community types}

In general, cutblocks provide only a limited source of forage for domestic livestock in the Upper Foothills subregion. The Brule stock association, Robb head tax permit and Upper James allotment are examples where the livestock rely principally on the forage within harvested cutblocks. On average, cutblocks produce twice as much forage as deciduous stands and nearly 3 times the forage as coniferous stands. In the Brule stock association, forage production on the cutblocks averaged 3-5 times greater than the unharvested white spruce dominated forest.

Two of the cutblock community types in this guide were described from the Brule stock association. These are the juniper/ hairy wildrye (UFF1) and rose/ hairy wildrye (UFF2) c.t.. Both of these types have very little growth of regenerating trees and resemble native grasslands (Figure 7).

Other cutblock community types were described on moister sites throughout the Solomon valley. These communities represent areas that were harvested 30-40 years ago. Currently, they are important sources of forage for domestic livestock throughout the area.

One burn community type was described from the Solomon valley. This burn occurred on an $\mathrm{Se}-\mathrm{Fa} /$ willow community approximately 10 years ago. The site was located in an areathat had nutrient rich seepage that made it very productive for horses grazing the area.

In the Upper James and Wilson creek allotments west of Sundre, harvesting of lodgepole pine dominated sites produces fireweed/hairy wildrye dominated communities on south and west facing slopes. On the more northern aspects in this area, the cutblocks tended to be dominated by moss to form the Pl-Sw/ moss community type. Livestock preferred to graze the fireweed/ hairy wildrye dominated cutblocks.

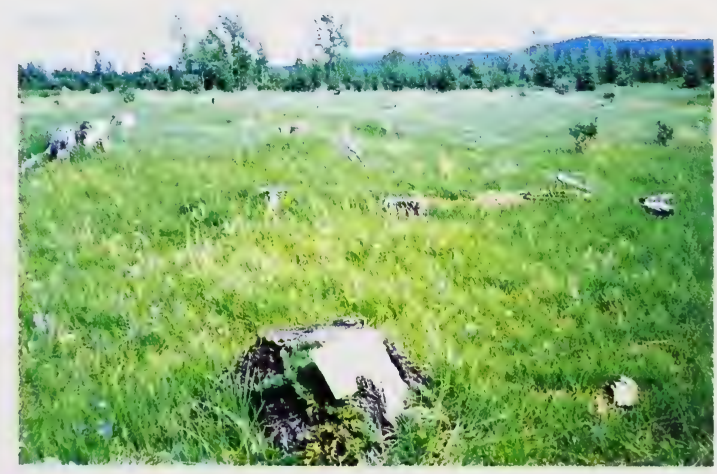

Figure 7. The juniper/ hairy wildrye community type (UFF1) results from the harvesting of a Sw/ juniper community. 
1

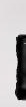

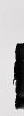

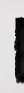

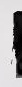

1

1

I

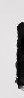

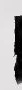

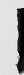

1

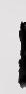

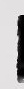

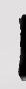

1

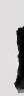

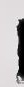

1 


\section{UFF1. Juniper/ Hairy wildrye \\ (Juniperus horizontalis/ Elymus innovatus)}

$\mathrm{n}=4$ This community represents a harvested Sw/ juniper community along the north shore of Brule lake. It is characteristic of the fine-textured, calcareous loess deposits, which have blown down the Athabasca river valley from Jasper National Park. The soils of this community have a high $\mathrm{pH}(8)$ which supports a good cover of hairy wildrye. This community type is extremely slow growing. When harvested, the cutblocks resemble native grasslands.

This community is very similar to the rose/ hairy wildrye community, but appears to be in a later successional stage. This community type was described in older cutblocks ( $35 \mathrm{yrs)}$ than the rose/ hairy wildrye community type (UFF2). As succession occurs on these cutblocks it appears that juniper and grass cover increase, causing a corresponding increase in forage production.

Plant Composition CaNopy COVER(\%). Mean Range Const.

TREES

WHITE SPRUCE

(Picea glauca)

BALSAM POPLAR

$\begin{array}{llll}\text { (Populus balsamifera) } & 8 & 0-15 & 75\end{array}$

ASPEN

(Populus tremuloides) . $6 \quad 0-15 \quad 50$

UNDERSTORY TREES

WHITE SPRUCE

(Picea glauca)

BALSAM POPLAR

(Populus balsamifera) $\quad 1 \quad 0-1 \quad 25$

ASPEN

(Populus tremuloides) $\quad 1 \quad 0-2 \quad 50$

SHRUBS

SHRUBBY CINQUEFOIL

(Potentilla fruticosa)

CREEPING JUNIPER.

(Juniperus horizontalis)

PRICKLY ROSE

(Rosa acicularis)

WILLOW

(Salix spp.)

BEARBERRY

(Arctostaphylos uva-ursi) 9

FORBS

SHOWY LOCOWEED

(Oxytropis splendens)

NORTHERN HEDYSARUM

(Hedysarum boreale)

NORTHERN BEDSTRAW

(Galium boreale)

GRASSES
HAIRY WILDRYE

(Elymus innovatus) $\quad 12 \quad 3-24 \quad 100$

BLUNT SEDGE

(Carex obtustata)

$6 \quad 0-15 \quad 75$

SLENDER WHEATGRASS

(Agropyron trachycaulum)2

$0-4 \quad 50$

\section{ENVIRONMENTAL VARIABLES}

MOISTURE REGIME:

SUBMESIC

NUTRIENT REGIME:

SUBMESOTROPHIC

ELEVATION:

1036-1066 M (1046 M)

SOIL DRAINAGE:

WELL

ASPECT:

SOUTH

SLOPE:

$0-5 \%$

\section{FORAGE PRODUCTION(KG/HA)}

GRASS: $520(268-866)$

FORBS: 697 (124-1538)

SHRUBS: $267(12-450)$

TOTAL: 2089 (592-3732)

\section{SUGGESTED GRAZING CAPACITY}

0.4 HAAUM OR $1.0 \mathrm{AC} / \mathrm{AUM}$ 


\section{UFF2. Rose/ Hairy wildrye \\ (Rosa acicularis/ Elymus innovatus)}

$\mathrm{n}=10$ This community type represents a Sw/ juniper community that was harvested 20 years ago. It is very similar to the previously described juniper/ hairy wildrye community (UFF1), but lacks the cover of juniper. It appears that harvesting disturbance causes juniper to decline in cover. As succession occurs, juniper and grass density increase, causing forage productivity to increase. The site conditions are so harsh it appears that grass cover has to undergo succession onto the site.

\section{Plant Composition Canopy Cover(\%)} MEAN RANGE CONST.

\section{TREES}

WHITE SPRUCE

(Picea glauca)-

BÄLSAM POPLAR

(Populus balsamifera)

\section{ASPEN}

(Populus tremuloides)

UNDERSTORY TREES

WHITE SPRUCE

(Picea glauca)

BALSAM POPLAR

(Populus balsamifera)

ASPEN

(Populus tremuloides)

SHRUBS

SHRUBBY CINQUEFOIL

(Potentilla fruticosa)

CREEPING JUNIPER.

(Juniperus horizontalis)

PRICKLY ROSE

(Rosa acicularis)

WILLOW SPP.

(Salix spp.)

BEARBERRY

(Arctostaphylos uva-ursi)

FORBS

WHITE CAMAS

(Zigadenus elegans)

SHOWY LOCOWEED

(Oxytropis splendens)

NORTHERN HEDYSARUM

(Hedysarum boreale)

(Galium boreale)

DANDELION

(Taraxacum officinale)

GRASSES
HAIRY WILDRYE

(Elymus innovatus) $\quad 24 \quad 4-40 \quad 100$

BLUNT SEDGE

$\begin{array}{llll}\text { (Carex obtustata) } & 2 & 0-7 & 60\end{array}$

SLENDER WHEATGRASS

(Agropyron trachycaulum) $4 \quad 0-10 \quad 70$

\section{ENVIRONMENTAL VARIABLES}

MOISTURE REGIME: MESIC

NUTRIENT REGIME: MESOTROPHIC

ELEVATION: $1036 \mathrm{M}$

SOIL DRAINAGE: WELL

ASPECT: SOUTHERLY

SLOPE:

$2-10 \%$

\section{FORAGE PRODUCTION(KG/HA)}

GRASS: $723(212-1514)$

FORBS: $388(126-756)$

SHRUBS: $132(2-454)$

TOTAL: $1243(540-2360)$

\section{SUGGESTED GRAZING CAPACITY}

0.7 HAAUM OR 1.6 AC/AUM 


\section{UFF2a. Fireweed/ Hairy wildrye \\ (Epilobium angustifolium/ Elymus innovatus)}

$\mathbf{n = 2 2}$ This community type represents a $\mathrm{P} 1 /$ moss community that was harvested 5-7 years ago. This community type was described on south and west facing slopes throughout the area. On more northerly aspects, moss dominates the understory of these cutblocks. Cutblocks can be an important source of forage for domestic livestock. They produce on average twice as much as deciduous stands, and nearly three times more than conifer stands. It must be remembered that this increase in forage is only temporary. As the cutblock undergoes succession there is a corresponding drop in production.

\section{Plant COMPOSITION CANOPY COVER(\%) MEAN RANGE CONST.}

UNDERSTORY TREES

LODGEPOLE PINE'

(Pinus contorta)

ASPEN

(Populus tremuloides) $1 \quad 0-2 \quad 23$

SHRUBS

PRICKLY ROSE

(Rosa acicularis)

WILLOW SPP.

(Salix spp.)

BUNCHBERRY

(Cornus canadensis) $\quad 1 \quad 0-1 \quad 73$

FORBS

FIREWEED

(Epilobium angustifolium) $5 \quad 0-7 \quad 91$

SHOWY ASTER

$\begin{array}{llll}\text { (Aster conspicuus) } & 1 & 0-7 & 46\end{array}$

NORTHERN BEDSTRAW

(Galium boreale) $\quad 1 \quad 0-1 \quad 27$

GRASSES

HAIRY WILDRYE

(Elymus innovatus)

$12 \quad 0-16 \quad 96$

SEDGE

(Carex spp)

$3 \quad 0-9 \quad 91$

PINEGRASS

(Calamagrostis rubescens) 1

\section{ENVIRONMENTAL VARIABLES}

MOISTURE REGIME:

SUBMESIC

NUTRIENT REGIME:

MESOTROPHIC

ELEVATION:

$1390-1700 \mathrm{M}(1513 \mathrm{M})$

SOIL DRAINAGE:

WELL

ASPECT:

VARIABLE

SLOPE:

$2-30 \%$

\section{FORAGE PRODUCTION(KG/HA)}

GRASS: 704 (566-842)

FORBS: $511(178-844)$

TOTAL: 1215 (744-1686)

\section{SUGGESTED GRAZING CAPACITY}

$0.7 \mathrm{HA} A U M$ OR $1.7 \mathrm{AC} / \mathrm{AUM}$ 


\section{UFF3. White spruce/ Horsetail/ Kentucky bluegrass \\ (Picea glauca/ Equisetum arvense/ Poa pratensis)}

$\mathrm{n}=1$ This community type represents a Sw/ Horsetail community that was harvested $30-40$ years ago along the banks of Moosehorn creek east of Rock Lake. These cutblocks are an important source of forage for domestic livestock and have been extensively utilized by cattle throughout the summer months. The high moisture and nutrient content of the sites make them extremely productive. Once invaded by agronomic species (Kentucky bluegrass and clover) they are extremely palatable to livestock.

\section{PLANT COMPOSITION CANOPY COVER(\%) MEAN RANGE CONST.}

\section{TREES}

WHITE SPRUCE

(Picea glauca)-

SHRंUBS

WILLOW

(Salix spp.)

PRICKLY ROSE

(Rosa acicularis)

FORBS

DANDELION

(Taraxacum officinale)

TALL LARKSPUR

(Delphinium glaucum) 5 - 100

DEWBERRY

(Rubus pubescens)

TALL LUNGWORT

(Mertensia paniculata) $5 \quad$ - 100

YARROW

(Achillea millefolium)

HORSETAIL

(Equisetum arvense) $\quad 2 \quad-\quad 100$

GRASSES

HAIRY WILDRYE

(Elymus innovatus)

KENTUCKY BLUEGRASS

(Poa pratensis)

SLENDER WHEATGRASS

(Agropyron trachycaulum) 3

\section{ENVIRONMENTAL VARIABLES}

MOISTURE REGIME:

SUBHYGRIC

NUTRIENT REGIME:

PERMESOTROPHIC

ELEVATION:

$1350 \mathrm{M}$

SOIL DRANAGE:

MODERATELY WELL

ASPECT:

NORTH

SLOPE:

$1 \%$

FORAGE PRODUCTION(KG/HA)

GRASS: 498

FORBS: 2378

TOTAL: 2876

\section{SUGGESTED GRAZING CAPACITY \\ 0.3 HAAUM OR 0.7 AC/AUM}




\section{UFF4. White spruce/ Moss \\ (Picea glauca/ Moss spp.)}

$\mathbf{n}=1$ This community type represents a Sw/moss community that was harvested $30-40$ years ago along the banks of West Solomon Creek. The regeneration on this cutblock is to subalpine fir which is similar to the understory of the Sw/ moss (UFE10) community that was harvested in the same area. This community is an important source of forage for wintering horses. The open canopy cover allows for a greater abundance of forbs and grasses in the understory. As the community continues to undergo succession and the canopy becomes denser there will be a corresponding drop in available forage.

PLANT Composition CANopy COVER(\%)

MEAN RANGe CONST.

TREES

WHITE SPRUCE

(Picea glauca)

SUBALPINE FIR

(Abies lasiocarpa)

SHRUBS

WILLOW

(Salix spp.)

RIVER ALDER

(Alnus tenuifolia)

BUNCHBERRY

(Cornus canadensis) $\quad 1 \quad$ - 100

FORBS

FIREWEED

(Epilobium angustifolium)4 $\quad$ - 100

PEAVINE

(Lathyrus ochroleucus) $\quad 1 \quad$ - 100

Moss

FEATHER MOSS

(Pleurozium scherberi)

\section{ENVIRONMENTAL VARIABLES}

MOISTURE REGIME:

MESIC

NUTRIENT REGIME:

MESOTROPHIC

ELEVATION:

$1300 \mathrm{M}$

SOIL DRAINAGE:

MODERATELY WELL

ASPECT:

EAST

SLOPE:

$10 \%$

FORAGE PRODUCTION(KG/HA)

GRASS: 428

FORBS: 476

SHRUBS: 78

TOTAL: 982

\section{SUGGESTED GRAZING CAPACITY \\ 0.9 HAAUM OR 2.1 AC/AUM}




\section{UFF4a. Lodgepole pine-White spruce/ Moss \\ (Pinus contorta-Picea glauca/Moss spp.)}

$\mathrm{n}=6$ This community type represents a Sw/moss or Lodgepole pine community that was harvested 5-10 years ago. These moss dominated cutblocks tend to occupy north aspects where the climatic conditions are cooler and moister. Livestock do not prefer to graze these sites.

Plant Composition CANopy Cover(\%) MEAN RANGe CONST.

\section{TREES}

WHITE SPRUCE

(Picea glauca)

LODGEPOLE PINE

(Pinus contorta)

UNDERSTORY TREES

WHITE SPRUCE

(Picea glauca)

LODGEPOLE PINE

(Pinus contorta)

SHRUBS

WILLOW

(Salix spp.)

ROSE

(Rosa acicularis)

BUNCHBERRY

(Cornus canadensis)

FORBS

FIREWEED

(Epilobium angustifolium) 1

HORSETAIL

(Equisetum arvense) $\quad 5 \quad 0-18 \quad 50$

GRASS

HAIRY WILDRYE

(Elymus innovatus)

MARSH REEDGRASS

(Calamagrostis canadensis)

MosS

FEATHER MOSS

(Pleurozium scherberi) $1 \quad 0-3 \quad 50$

STAIR STEP MOSS

(Hylocomium splendens)

$3 \quad 0-15 \quad 17$

$10 \quad 0-25 \quad 50$

$2 \quad 0-8 \quad 50$

$2 \quad 0-10 \quad 17$

$1 \quad 0-2 \quad 67$

$1 \quad 0-2 \quad 100$

$2 \quad 0-10 \quad 50$

$0-5 \quad 50$

0-13 100

$0-8 \quad 33$

$0-15 \quad 67$

\section{ENVIRONMENTAL VARIABLES}

MOISTURE REGIME:

MESIC

NUTRIENT REGIME:

MESOTROPHIC

ELEVATION:

1335-1572 M (1414 M)

SOIL DRAINAGE:

MODERATELY WELL

ASPECT:

VARIABLE

SLOPE:

$0-16 \%$

FORAGE PRODUCTION(KG/HA)

TOTAL: 450

\section{SUGGESTED GRAZING CAPACITY}

NON-USE 


\section{UFF5. River alder-Willow/ Fireweed-Cow parsnip (Alnus tenuifolia-Salix spp./ Epilobium angustifolium-Heracleum lanatum)}

$\mathrm{n}=1$ This community type represents a Engelmann $\mathrm{x}$ White spruce-Subalpine fir community that was burned 10 years ago. The site was located within a nutrient rich seepage area, which favoured the growth of cow parsnip, fireweed and horsetail. The lack of tree canopy cover and moisture and nutrient regime of the area made the site very productive for domestic livestock. This site was extensively utilized by horses throughout the winter and summer months

\section{PLANT COMPOSITION CANOPY COVER(\%)} MEAN RANGe CONST.

TREES

WHITE SPRICE

(Picea glauca)

ASPEN

(Populus tremuluides) $5 \quad-100$

SHRLBS

WILLW

(Salix SPP)

RIVER ALDER

(Alnus ienuifolis)

FORBS

FIREWEED

(Epilobium angustufolium) 21

COW PARSNIP

(Heracleum lanasum) $13 \quad-\quad 100$

STINGING NETTLE

(Urtica dioica) $\quad 10 \quad-\quad 100$

WHITE GERANIUM

$5 \quad-100$

$5 \quad-100$

(Geranium richardsonii) $10 \quad-\quad 100$

TALLLUNGWORT

(Mertensia paniculata) $8 \quad-100$

HORSETAIL.

(Equisetum arvense)

GRASSES

SLENDER WHEATGRASS

(Agropyron trachycaulum)

MARSH REEDGRASS

(Calamagrostis

canadensis)

\section{ENVIRONMENTAL VARIABLES}

MOISTURE REGIME:

SUBHYGRIC

NUTRIENT REGIME:

PERMESOTROPHIC

ELEVATION:

$1200 \mathrm{M}$

SOIL DRAINAGE:

MODERATELY WELL

ASPECT:

EAST

SLOPE:

$20 \%$

\section{FORAGE PRODUCTION(KG/HA)}

GRASS: 122

FORBS: 3034

TOTAL: 3156

SUGGESTED GRAZNG CAPACITY

0.3 HAAUM OR 0.6 AC/AUM 


\section{UFF6. Aspen/ Fireweed \\ (Populus tremuloides/ Epilobium angustifolium)}

$\mathrm{n}=1 \quad$ This community type represents a Pl-Sw/ bunchberry community that was harvested near the Robb area. The regeneration of this community back to aspen indicates that this particular cutblock is transitional to the Lower Foothills subregion. Indeed, the Robb area is on the border between the Upper and Lower Foothills subregions. This community type is highly productive for domestic livestock. Harvesting the trees allows the grasses and forbs to grow, increasing the forage productivity.

\section{Plant Composition Canopy Cover(\%)}

MEAN RANGE CONST.

\section{UNDERSTORY TREES \\ WHITE SPRUCE}

(Picea glauca) -

ASPEN

(Populus tremuloides) $6 \quad-100$

SHRUBS

PRICKIY ROSE.

(Rosa acicularis) $\quad 6 \quad-100$

GREEN ALDER

(Alnus crispa)

DEWBERRY

(Rubus pubescens)

FORBS

FIREWEED

(Epilobium angustifolium) 52

HORSETAIL

(Equisetum arvense) $\quad 9 \quad-100$

HEART LEAVED ARNICA

(Arnica cordifolia)

TALL LUNGWORT

(Mertensia paniculata) $2 \quad-100$

GRASSES

SEDGE spp.

(Carex sp.)

MARSH REEDGRASS

(Calamagrostis

canadensis)

$7 \quad-100$

$3 \quad-100$

100

100

100

$13 \quad-\quad 100$

\section{ENVIRONMENTAL VARIABLES}

MOISTURE REGIME:

MESIC

NUTRIENT REGIME :

PERMESOTROPHIC

ELEVATION:

$1091 \mathrm{M}$

SOIL DRAINAGE :

MODERATELY WELL

ASPECT:

NORTH

SLOPE:

$5 \%$

\section{FORAGE PRODUCTION(KG/HA)}

GRASS: 540

FORBS: 1520

SHRUBS: 150

TOTAL: 2210

\section{'Suggested Grazing Capacity} 0.8 HAAUM OR 1.8 AC/AUM 


\section{UFF7. Aspen/ Blueberry-Bearberry/ Hairy wildrye}

(Populus tremuloides/Vaccinium myrtilloides-Arctostaphylos uva-ursi/ Elymus innovatus) $\mathrm{n}=2$ This community type represents a PV bog cranberry community (UFE1) that was harvested near the Robb area. The ecological conditions of this site are drier with a poorer nutrient regime. Regeneration of the trees will be much slower than the Aw/ fireweed community type which was described previously. Harvesting of the pine overstory allows grasses and forbs to flourish. This provides a good forage base for domestic livestock. Caution must be used when grazing cutblocks that the stocking rate is not too high to limit the growth of regenerating trees.

\section{Plant COMPOSITION Canopy COVER(\%)}

MEAN RANGe CONST.

\section{UNDERSTORY TREES}

WHITE SPRUCE

(Picea glauca)

$1 \quad 0-1 \quad 50$

ASPEN

(Populus tremuloides)

LODGEPOLE PINE

(Pinus contorta)

SHRUBS

BLUEBERRY

(Vaccinium myrtilloides) $11 \quad$ T-22 100

BOG CRANBERRY

(Vaccinium caespitosum) $2 \quad 0-3 \quad 50$

FORBS

FIREWEED

(Epilobium angustifoliun) $1 \quad 0-2 \quad 50$

LINDLEY'S ASTER

(Aster ciliolatus)

$2 \quad 0-4 \quad 50$

STRAWBERRY

$\begin{array}{llll}\text { (Fragaria virginiana) } & 2 & \mathrm{~T}-3 & 100\end{array}$

YARROW

$\begin{array}{llll}\text { (Achillea millefolium) } & 1 & 0-2 & 50\end{array}$

GRASSES

INDIAN RICEGRASS

(Oryzopsis pungens)

HAIRY WILDRYE

(Elymus innovatus)
2

T-3

100

$6 \quad T-10 \quad 100$
ENVIRONMENTAL VARIABLES

MOISTURE REGIME :

SUBMESIC

NUTRENT REGIME:

MESOTROPHIC

ELEVATION:

$1091 \mathrm{M}$

SOIL DRAINAGE :

WELL

ASPECT:

EAST

SLOPE:

$3 \%$

FORAGE PRODUCTION(KG/HA)

GRASS: 300

FORBS: 310

SHRUBS: 285

TOTAL: 895

\section{SUGGESTED GRAZING CAPACITY 2.0 HAAUM OR 4.6 AC/AUM}




\section{UFF8. Kentucky bluegrass-Creeping red fescue/ Clover (Poa pratensis-Festuca rubra/ Trifolium spp.)}

$\mathbf{n}=\mathbf{5}$ This community type represents cutblocks that have been heavily grazed by livestock. Heavy livestock grazing favours the growth of the invaders Kentucky bluegrass and timothy. The grazing pressure which favours the growth of these grass species is usually detrimental to the growth of trees. Cattle damage to the conifer trees is usually trampling damage which scars the trees and breaks the stem.

Plant Coniposition Canopy COVER(\%)

MEAN RANGe CONST.

SHRL'BS

ROSE

(Rosa acicuicaris) $\quad 1 \quad 0-4 \quad 20$

FORBS

FIREWEED

(Epilobium angustífilum) $1 \quad 0-3 \quad 80$

CLOVER

(Trifolum spp) $\quad 15 \quad 0-48 \quad 80$

STRAHBERRY

(Fragariasirginans) $\quad 1 \quad 0-2 \quad 40$

YARROW

$0-T \quad 60$

GRASSES

TIMOTHY

(Phleum pratense)

HAIRY WILDRYE

(Elymus innovatus) $\quad 1 \quad 0-3 \quad 40$

KENTUCKY BLUEGRASS

$\begin{array}{llll}\text { (Poapratensis) } & 15 & 0-67 \quad 80\end{array}$

CREEPING RED FESCUE

(Festuca rubra)
ENVIRONMENTAL VARIABLES

MOISTURE REGIME:

SUBMESIC

NUTRIENT REGIME:

SUBMESOTROPHIC

ELEVATION:

$1435-1480 \mathrm{M}(1453 \mathrm{M})$

SOIL DRAINAGE:

MODERATELY WELL

ASPECT:

VARIABLE

SLOPE:

$0-12 \%$

FORAGE PRODUCTION(KG/HA)

GRASS: 932

TOTAL: 932

\section{SUGGESTED GRAZING CAPACITY} 2.0 HAAUM OR 4.6 AC/AUM 


\section{Literature cited}

Bailey, A.W., M.G. Willoughby, R. Johansen and S. Smith. Management of Yukon

Rangelands. Renewable Resources, Yukon Territorial Government, Whitehorse, Yukon. 55pp. ISBN-1-55018-138-6.

Bork, E. 1990. Clearwater allotment: Prescribed burn vegetation survey, evaluation, and discussion. Range Management Section, Alberta Forest Service, Edmonton, Alta. 29pp.

Bork, E. 1994. Ecological classification and management of native ranges in Willmore Wilderness Park. Alberta Environmental Protection. Forest Management Division. Edmonton, Alta. Pub. no. T/282. 65pp.

Beckingham, J. 1994. Field guide to the ecosystems of Northern Alberta. Environmental Protection, Land and Forest Services, Edmonton, Alta. 352pp.

Beckingham, J.D., I.G.W. Corns and J.H. Archibald. 1996. Field guide to ecosites of WestCentral Alberta. Nat. Resour. Can., Can. For. Serv., Northwest Reg., North. For. Cent., Edmonton, Alberta. Spec. Rep. 9.

Corns, I.G.W. and P.L. Achuff. 1982. Vegetation type descriptions. In Holland, W.D. and G.M. Coen. Ecological (Biophysical) Land Classification of Banff and Jasper National Parks. Vol II. Soil and Vegetation Resources. Environment Canada and Alberta Institute of Pedology, University of Alberta. Pub. no. SS-82-44. 540pp.

Corns, I.G.W. and R.M. Annas. 1986. Field guide to forest ecosystems of West-Central Alberta. Northern Forestry Center, Canadian Forestry Service, Edmonton, Alta. 251pp.

Daubenmire, R. 1952. Forest vegetation of Northern Idaho and adjacent Washington and its bearing on concepts of vegetation classification. Ecol. Mongr. 22: 301-330.

Daubenmire, R. 1978. Plant Geography. Academic Press. New York. 338pp.

Dept. of Environmental Protection. 1994. Natural regions of Alberta. Alberta Environmental Protection. Edmonton, Alta. Pub. no. I/531. 18pp.

Gauch, H.G. 1982. Multivariate analysis in community ecology. Cambridge University Press, Cambridge, 298pp.

Lane, C. T., M.G. Willoughby and M.J. Alexander. 2000. Range plant community types and carrying capacity for the Lower Foothills subregion of Alberta. Alberta Environment and Alberta Agriculture Food and Rural Development. Edmonton. AB. Pub. no. T/532. 232pp. 
Mackinnon, A., J. Pojar, and R. Coupe. 1992. Plants of Northern British Columbia. Lone Pine Publishing, Edmonton, Alta. 345pp.

Mueggler, W.F. 1988. Aspen community types of the Intermountain Region. U.S.D.A. Intermoutain Research Station. INT-250. 133pp.

Rangeland Resource Information System. 1991. Outline and guide, a proposal. Range Management Section, Alberta Forest Service, Edmonton, Alta. 19pp.

Range Survey Manual. 1992. Range Management Section, Alberta Forest Service. Edmonton, Alta. 39pp.

Strong, W.L. and H.G. Anderson. 1980. Ecological land classification and evaluation reference manual. Resource Evaluation reference manual. Resource Evaluation Branch, Energy and Natural Resources. Edmonton, Alta. 160pp.

Strong, W.L. and K.R. Leggat. 1992. Ecoregions of Alberta. Alberta Forestry, Lands and Wildlife, Resource Information Branch, Edmonton, Alta. T/245. 77pp.

Strong, W.L. 1992. Ecoregions and Ecodistricts of Alberta. Alberta Forestry, Lands and Wildlife. Edmonton, Alta. Pub. no. T/244. 77pp.

Willoughby, M.G. 1992. Rangeland Reference Areas, Plant communities, ecology and response to grazing in Division 3. Forestry, Lands and Wildlife, Alberta Forest Service, Edmonton, Alta. T/269, 36pp.

Willoughby, M.G. 1995. The effect of grazing on deciduous plant communities in the Boreal Ecoprovince of Alberta. Fifth International Rangeland Congress. Salt Lake City Utah. pg. 608609.

Willoughby, M.G. 1999. Range plant community types and carrying capacity for the Subalpine and Alpine subregions of Alberta. Environmental Protection. Land and Forest Services. Edmonton, Alta. Pub. no. T/438. 109pp.

Willoughby, M.G. 2000. Guide to range plant communities and carrying capacity for the Dry and Central Mixedwood subregions of Alberta. Alberta Environment and Alberta Agriculture Food and Rural Development. Edmonton. AB. Pub. no. T/526. 187pp.

Willoughby, M.G. 2000. Rangeland Reference Areas: Species composition changes in the presence and absence of grazing and fire on the Rough fescue-Hairy wildrye dominated community types of the Upper Foothills subregion. Alberta Environment. Edmonton. AB. Pub. no. T/572. 30pp. 
Willoughby, M.G., M.J. Alexander and K.M. Sundquist. 2001. Range plant community types and carrying capacity for the Montane subregion. Alberta Environmental Protection. Edmonton. AB. Pub. no. T/589. $194 \mathrm{pp}$.

Youngblood, A. Community type classification of forest vegetation in young, mixed stands, Interior Alaska. U.S.D.A. Forest Service, Pacific Northwest Research Station. PNW-RP-458. $42 \mathrm{pp}$. 
I

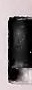

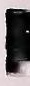

i

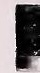

|

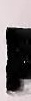

,

,

,

,

,

,

,

,

8

,

| 
National Library of Canada

Bibliothèque nationale du Canada

||||||||||||||||||||||||||||||||||||||||||||||||||||||||||||||||||||||||||||||||||||||||||||||||||||||

33286524766470 